OPEN ACCESS

Edited by: Jianping Liu, Beijing University of Chinese Medicine,

China

Reviewed by: Johanna Mahwahwatse Bapela, University of Pretoria, South Africa

Su Chunxiang,

Beijing University of Chinese Medicine,

China

${ }^{*}$ Correspondence:

Guo-Qing Zheng

gq_zheng@sohu.com

Yan Lin

linyanm@vip.sina.com

Specialty section:

This article was submitted to

Ethnopharmacology,

a section of the journal

Frontiers in Pharmacology

Received: 27 May 2019

Accepted: 24 June 2020

Published: 17 July 2020

Citation:

Jin $T-Y$, Rong $P-Q$, Liang $H-Y$, Zhang $P-P$, Zheng G- $Q$ and Lin $Y$

(2020) Clinical and Preclinical

Systematic Review of

Panax ginseng C. A. Mey and lts

Compounds for Fatigue.

Front. Pharmacol. 11:1031.

doi: 10.3389/fphar.2020.01031

\section{Clinical and Preclinical Systematic Review of Panax ginseng C. A. Mey and Its Compounds for Fatigue}

\author{
Ting-Yu Jin, Pei-Qing Rong, Hai-Yong Liang, Pei-Pei Zhang, Guo-Qing Zheng * \\ and Yan Lin*
}

Department of Neurology, The Second Affiliated Hospital and Yuying Children's Hospital of Wenzhou Medical University, Wenzhou, China

Background: Fatigue, as a complex, multidimensional symptom, is associated with many physical illnesses. Panax ginseng C. A. Mey (PG) is an important herbal drug which has been used for benefiting Qi for thousand years. Panax ginseng C. A. Mey and its compounds (PGC) possess various pharmacological activities, including anti-fatigue. Here, we conducted a systematic review of both randomized clinical trials (RCTs) and preclinical animal studies to investigate the efficacy and safety of PGC for fatigue.

Methods: Electronic searches were performed in 7 databases from the time of each database's inception to August 2019. The methodological quality of RCTs was assessed using 7-item checklist recommended by Cochrane Collaboration or by the CAMARADES 10item quality checklist. All the data were analyzed using Rev-Man 5.3 and Stata SE software.

Results: Eight eligible RCTs and 30 animal studies were identified. The risk of bias scores in RCTs ranged from 4/7 to $7 / 7$, and of animal studies varied from 4/10 to 7/10. Metaanalyses showed that PGC was superior to placebo according to their respective fatigue scales, heart rate recovery, and clinical effect $(P<0.05)$. There were a similar number of adverse effects between PGC and placebo group $(P>0.05)$. Meta-analyses showed that PGC can significantly decrease level of blood lactate, blood urea nitrogen, creatine kinase, malondialdehyde, and lactic dehydrogenase in serum, level of malondialdehyde in liver and level of gamma-aminobutyric acid, 5-hydroxytryptamine in brain tissue, and increase swimming time, level of glutathione peroxidase, glucose, superoxide dismutase in serum, level of glycogen and activity of superoxide dismutase, glutathione peroxidase, and catalase in skeletal muscle, level of hepatic glycogen in liver and level of dopamine, acetylcholine in brain tissue, compared with control $(P<0.05)$. Meta-analyses showed no significant difference in animal body weight between $P G C$ and control $(P>0.05)$.

Conclusion: The present findings supported, to a certain degree, that PGC can be recommended for routine use in fatigue. The possible mechanism of PGC resists fatigue, mainly through antioxidant stress, regulating carbohydrate metabolism, delaying the 
accumulation of metabolites, promoting mitochondrial function, neuroprotection, antiapoptosis, and regulating neurotransmitter disorder in central nervous system.

Keywords: ginseng, fatigue, randomized controlled trials, animal studies, systematic review

\section{INTRODUCTION}

\section{Description of the Condition}

Fatigue is a condition of lacking the energy and motivation in responding to physical activity, emotional stress, boredom or insufficient sleep (Bach et al., 2016). It is a complex, multidimensional symptom that is prevalent in the general population (Jason et al., 2010). The cause of fatigue is unknown. The severity of fatigue varies greatly among individuals. Although fatigue does not lead to death, it has negative impacts on many areas of daily life (Arring et al., 2018). "Feeling weak all over for much of the time" was regarded as one of the most important symptoms (Lewis and Wessely, 1992). Most physical illnesses are associated with fatigue, such as many chronic diseases, namely anaemia, emphysema, asthma, and arthritis (Chen, 1986). Fatigue is a clinical challenge because its study of etiology, risk factors, and pathophysiology are still at an early stage. The goal of treatment is to treat symptoms and improve outcomes rather than to provide clear treatment (Alraek et al., 2011).

\section{Description of the Intervention}

Different interventions have been used in treating fatigue (Whiting et al., 2001). Nowadays, treatments commonly focus on muscle pain, sleep disorders, and emotional symptoms. Cognitive Behavior Therapy (CBT), various forms of exercise, as well as enhancement of coping ability, are standard treatment options. In addition, caregiver prescribed or self-administered medication are still common (Jones et al., 2007). It is indicated that no universal western medicine treatment can be recommended (Collatz et al., 2016). Interventions which include CBT and graded exercise therapy have shown promising results (Whiting et al., 2001). However, patients seem to be skeptical about CBT, who claimed that CBT and graded exercise for fatigue were neither effective nor safe (Twisk and Corsius, 2018). Recently, various forms of complementary and alternative medicine (CAM) have been widely used in fatigue such as herbal medicine, cheirapsis, balanced nutrition, and acupuncture (Jones et al., 2007). In particular, Panax ginseng C.A. Mey (PG) has been a rising utilization in treating fatigue in Asia and elsewhere around world. Based on traditional Chinese medicine and herbal philosophy, PG is considered as an adaptation to help restore body balance (Arring et al., 2018). Panax ginseng C.A. Mey is believed to improve overall quality of life (QoL), including energy and vitality, particularly during times of fatigue or stress (Yennurajalingam et al., 2017).

\section{How the Intervention Might Work}

Panax ginseng C.A. Mey has direct effects on the central nervous system (CNS), including cognition, sleep disorders, depression, pain, and the ability to regulate inflammatory cytokines (Yennurajalingam et al., 2017). To date, numerous active compounds have been identified such as ginsenosides, ginseng polysaccharides, and ginseng protein. Ginsenosides, the most important ingredients of ginseng, have been proved with various pharmacological activities such as anti-fatigue, anti-oxidation, neuroprotection, antiinflammation, and anti-diabetes. Ginsenoside Rg3 (Rg3) is one of the most abundant ginsenosides. It may improve exercise performance and increase fatigue resistance by enhancing deacetylase activity of silent information regulator of transcription 1 (SIRT1) and inhibiting the transcriptional activity of p53 (Yang et al., 2018). Ginseng polysaccharides have anti-fatigue activity probably by mobilizing triglyceride (TG) or fat during exercise, or by changing the activities oflactic dehydrogenase $(\mathrm{LDH})$, malondialdehyde (MDA) and glutathione peroxidase (GPH-Px) to avoid lipid oxidation and protect corpuscular membrane (Zheng et al., 2017). Ginseng proteins could resist fatigue through retarding the accumulation of blood lactate (BLA) and blood urea nitrogen (BUN), enhancing hepatic glycogen levels, and improving the ability of antioxidant enzymes (Qi et al., 2014).

\section{Why It Is Important to Do This Review}

Panax ginseng C.A. Mey is one of the most widely used plant products worldwide which has been used in oriental countries for thousands of years (Zheng et al., 2017). Based on a comprehensive collection of clinical trials, systematic review can perform comprehensive analysis and statistical processing on qualified studies to form relatively reliable results, which can guide clinical decision-making. In addition, systematic review can solve the following clinical problems: research on the effectiveness of treatment, evaluation of diagnostic methods, prognosis estimation, analysis of the cost and benefit of treatment. Up to now, at least 2 systematic reviews have been conducted to evaluate efficacy and safety of Panax ginseng C. A. Mey and its compounds (PGC) for fatigue (Bach et al., 2016; Arring et al., 2018). However, the results of these reviews are inconclusive because of methodological flaws in their included primary studies. Cochrane group have developed an extensive set of guide lines for systematic reviews. These "not-so-good" studies were excluded with a strict process (Xie et al., 2013). In addition, the efficacy and mechanisms of PGC in fatigue animal models have not been systematically evaluated yet. Systematic review of animal researches is indispensable in the process of drug development and elucidation of the physiological and pathological mechanisms (Zheng et al., 2018). Preclinical research is the key to convert preclinical data into clinical data. In addition, systematic review of animal research is a more economic and ethical approach, which can integrate preclinical evidence, help reduce unnecessary sacrifice of laboratory animals, and prevent ineffective or less informative research (Zhou et al., 2019). As we all know, there is a gap between clinical research 
and clinical practice. More communication is needed between animal researchers and clinical researchers. Systematic review of animal experiments can lead to better collaboration between research groups and encourage the use of iterative methods to improve the relevance of animal models to clinical trial design. If the model cannot well simulate the clinical situation, it can be adjusted accordingly. In addition, as in human research, systematic review helps to identify and improve behavioral and reporting deficiencies in animal research Perel et al., 2007. Systematic review can effectively integrate preclinical comprehensive evidence and guide potential clinical translation. Thus, the aim of present study was to systematically summarize and critically evaluate the data from randomized control trials (RCTs) and animal studies of PGC for fatigue.

\section{METHODS}

\section{Search Strategy}

This study followed the PRISMA statement (Stewart et al., 2015). EMBASE, PubMed, Cochrane Library, China National Knowledge Infrastructure (CNKI), VIP database (VIP), China Biology Medicine Database (CBM) and Wangfang database were electronically searched from their inception to August 2019. The following keywords were used: "fatigue OR Lassitude lethargy OR exhaustion OR weariness OR tiredness" and "panax OR Ginseng OR renshen" in Chinese or in English. All searches were limited to animal studies and clinical trials.

\section{Eligibility Criteria}

The prespecified inclusion criteria of RCTs listed below: (1) RCTs that evaluated the effectiveness and safety of PGC for fatigue; (2) the Cochrane risk of bias (ROB) tool met at least 4 out of the 7 domains; (3) Subjects had chronic fatigue syndrome (CFS) or healthy adults after exercise; Subjects were classified as CFS-like according to Evaluation and Classification of Unexplained Chronic Fatigue (ECUCF) (Fukuda et al., 1994); (4) PGC as monotherapy was used as an intervention in the treatment group, and interventions for control group were placebo or vehicle treatment; (5) The primary outcome measures were scales of fatigue and/ or objective evaluation criteria (e.g. physical performance, biochemical parameters). The secondary outcome measures were clinical effect according to fatigue scales and adverse events. The exclusion criteria were prespecified as follows: (1) fatigue caused by a medical condition, or withdrawal from medicines or substance; (2) duplicate publications and no available data.

The inclusion criteria of animal studies were prespecified as follows: (1) PGC for fatigue animal models was established by forced movement; (2) The interventions of treatment group were PGC at any dose and control group were nonfunctional liquid (normal saline) or no treatment; and (3) The primary outcome measures were forced movement time and/or serum biochemical parameters and/or skeletal muscle biochemical parameters and/or liver biochemical parameters and/or brain tissue biochemical parameters. The secondary outcome measures were body weight, organ index (organ weight/body weight) and possible mechanisms of PGC for anti-fatigue. The exclusion criteria were predefined as follows: (1) not fatigue model; (2) combined use of other drugs; and (3) no available data, duplicate publications, and lack of control group.

\section{Data Extraction}

Two independent researchers extracted the details from the included RCTs and animal studies according to two standardized data extraction forms, respectively. There are many manners for including outcomes, such as peak time point, last time point, and same time point. There is undeniable that any manner will lead to bias. In order to minimize bias, inclusion criteria were prespecified as follows: The result of the peak time point was included when the data were expressed at different times. If meta-analysis data were lost or expressed graphically, we would try to contact the author for more information. When no response was received, we used digital ruler software or exclusion software to measure data from charts. If the data in the primary RCT were missing or merely illustrated graphically, an effort was launched to obtain further information through contacting the authors. If failed, the digital ruler software was used for measuring data from the graphs or excluded.

\section{Quality Assessment}

The methodological quality of the included RCTs was evaluated by using the Cochrane Collaboration's tool. The RoB of the included animal studies was assessed using 10-item quality checklist of the Collaborative Approach to Meta-Analysis and Review of Animal Data from Experimental Studies (CAMARADES) with minor modification. Divergences were well settled by correspondence author (GZ).

\section{Statistical Analysis}

Analysis was conducted with RevMan 5.3 and Stata SE software. Continuous outcomes were presented as mean difference (MD) or standardized mean difference (SMD) with 95\% confidence interval (CI). Dichotomous outcomes were presented risk ratio (RR) or odds ratio (OR) with 95\% CI. Probability values $\mathrm{P}<0.05$ were considered significant. In order to estimate heterogeneity across studies, we used $\mathrm{I}^{2}$ - statistic test. An $\mathrm{I}^{2}$ value greater than $50 \%$ was considered as having substantial heterogeneity. When substantial heterogeneity was not observed, the fixed-effects model was reported. On the contrary, the random-effects model was reported. Simultaneously, considering the differences in subjects, interventions, and treatments, we used the Z-test for subgroup analysis. $\mathrm{P}<0.05$ was considered to be statistically significant. If an outcome contained more than 10 RCTs, funnel plots, and Egger's test were used to examine publication bias.

\section{RESULTS}

\section{Study Selection}

A total of 1331 relevant literatures were retrieved from the database, of which 667 were considered duplicates. Of the remaining 664 articles, qualified RCTs and animal experiments should be selected separately. For RCTs, 362 articles were 
eliminated because of reviews, case report, or animal studies. After scanning the remaining 302 full-text articles, 294 studies were excluded by reasons that they were (1) combined with other disease; (2) combined with other herbal treatment(s) in the intervention group; (3) no data available; (4) not real RCTs or quasi-RCTs; or (5) with the less than 4 domains "yes" according to the Cochrane RoB tool. Eventually, 8 RCTs were selected (Figure 1A). For animal studies, 243 studies including clinical trials, case reports or review articles were excluded. Through fulltext evaluation of the remaining 421 studies, 391 were excluded for at least one of the following reasons: (1) lack of control group; (2) inappropriate fatigue model; (3) combined with other herbal treatment(s) in the intervention group; (4) unavailable data. Ultimately, 30 animal studies were included (Figure 1B).

\section{Study Characteristics Randomized Control Trials}

All involved studies were published in English. All 8 studies (Gal et al., 1996; Engels et al., 1996; Engels et al., 2001; Engels et al., 2003; Hartz et al., 2004; Hyeong-Geug et al., 2013; Kim et al., 2016; Lee et al., 2016) were RCTs, which involved a total of 678 participants, and the sample size ranged from 19 to 218 . Five RCTs (Gal et al., 1996; Hartz et al., 2004; Hyeong-Geug et al., 2013; Kim et al., 2016; Lee et al., 2016) involved 615 participants with CFS. The diagnostic criteria of patients with CFS were based on ECUCF. The rest of 3 RCTs (Engels et al., 1996; Engels et al., 2001; Engels et al., 2003) involved 63 healthy adults after exercise. They were published from 1996 to 2016 and conducted in America ( $\mathrm{n}=4$ ) (Engels et al., 1996; Engels et al., 2001; Engels et al., 2003; Hartz et al., 2004), South Korea $(n=3)$ (Hyeong-Geug et al., 2013; Kim et al., 2016; Lee et al., 2016), and France $(n=1)$ (Gal et al., 1996). The duration of treatment ranged from 4 to 8 weeks. They used PGC as intervention of experimental group. The placebo-control was used in all 8 studies. Five studies (Engels et al., 1996; Engels et al., 2001; Engels et al., 2003; Hyeong-Geug et al., 2013; Kim et al., 2016) used objective evaluation criteria, including metabolic response and physical performance as outcome measure. Five studies (Gal et al., 1996; Hartz et al., 2004; Hyeong-Geug et al., 2013; Kim et al., 2016; Lee et al., 2016) used self-reported fatigue measures, including Rand Vitality Index (RVI), Mood and Anxiety Symptom Questionnaire (MASQ), fatigue duration, checklist individual strength (CIS), Numeric Scale (NRS), Visual Analogue Scale (VAS), Visual Analogue Fatigue Scale (VAFS), Revised Piper Fatigue Scale (RPFS), Short-Form Health Survey (SF-36), Fatigue score, and clinical effect as outcome measure. The general characteristics of the 8 included articles were illustrated in Tables $\mathbf{1}$ and $\mathbf{2}$.

\section{Animal Studies}

Nine studies (Choi et al., 2011; Hwang et al., 2014; Qi et al., 2014; Wang et al., 2014; Oh et al., 2015; Bao et al., 2016; Ma et al., 2017; Zheng et al., 2017; Delgado et al., 2019) were published in English and 21 studies (Feng et al., 2009a; Feng et al., 2009b; Feng et al., 2009c; Li et al., 2009; Pan et al., 2009; Feng et al., 2010a; Feng et al., 2010b; Feng et al., 2010c; Pan et al., 2010a; Pan et al., 2010b; Xu et al., 2010; Chen and Li, 2011; Gao et al., 2011; Xu et al., 2011; Song et al., 2013; Zhao et al., 2014; Liu et al., 2015; Wang et al., 2015; Liu, 2016; Shi et al., 2016; Yao, 2016) were published in Chinese between 2005 and 2019. All 30 studies involved a total of 2249 animals. Twelve studies (Feng et al., 2009a; Feng et al., 2009b; Feng et al., 2009c; Pan et al., 2009; Feng et al., 2010a; Feng et al., 2010b; Feng et al., 2010c; Pan et al., 2010a; Pan et al., 2010b; Xu et al., 2010; Chen and Li, 2011; Liu, 2016) used Sprague Dawley (SD) rats; 1 study (Song et al., 2013) used Wistar rats; 8 studies (Choi et al., 2011; Hwang et al., 2014; Wang et al., 2014; Oh et al., 2015; Bao et al., 2016; Ma et al., 2017; Zheng et al., 2017; Delgado et al., 2019) used Institute of Cancer Research (ICR) mice; 6 studies (Li et al., 2009; Xu et al., 2011; Zhao et al., 2014; Qi et al., 2014; Wang et al., 2015; Shi et al., 2016) used Kunming (KM) mice; 3 studies (Gao et al., 2011; Liu et al., 2015; Yao, 2016) used unknown breed mice. Twentyseven studies (Feng et al., 2009a; Feng et al., 2009b; Feng et al., 2009c; Li et al., 2009; Pan et al., 2009; Feng et al., 2010a; Feng et al., 2010b; Feng et al., 2010c; Pan et al., 2010a; Pan et al., 2010b; Xu et al., 2010; Chen and Li, 2011; Gao et al., 2011; Choi et al., 2011; Song et al., 2013; Hwang et al., 2014; Wang et al., 2014; Liu et al., 2015; Wang et al., 2015; Oh et al., 2015; Bao et al., 2016; Liu, 2016; Shi et al., 2016; Yao, 2016; Ma et al., 2017; Zheng et al., 2017; Delgado et al., 2019) used male animals, 2 studies (Qi et al., 2014; Zhao et al., 2014) used both female and male rats, and 1 study (Xu et al., 2011) did not mention gender of animals. The weight of adult rats varied between 160 and $260 \mathrm{~g}$ and mice between 16 and $28 \mathrm{~g}$. Anesthetic was mentioned in 1 study (Chen and Li, 2011). Ginsenosides were used in 18 studies (Feng et al., 2009a; Feng et al., 2009b; Feng et al., 2009c; Li et al., 2009; Pan et al., 2009; Feng et al., 2010a; Feng et al., 2010b; Feng et al., 2010c; Pan et al., 2010a; Pan et al., 2010b; Xu et al., 2010; Chen and Li, 2011; Song et al., 2013; Liu et al., 2015; Oh et al., 2015; Wang et al., 2015; Liu, 2016; Yao, 2016), ginseng in 6 studies (Choi et al., 2011; Gao et al., 2011; Hwang et al., 2014; Zhao et al., 2014; Shi et al., 2016; Ma et al., 2017), ginseng oligopeptides in 2 studies (Bao et al., 2016; Delgado et al., 2019), ginseng polysaccharides in 2 studies (Wang et al., 2014; Zheng et al., 2017) and ginseng protein in 2 studies (Xu et al., 2011; Qi et al., 2014). Thirteen fatigue models (Feng et al., 2009a; Feng et al., 2009b; Feng et al., 2009c; Pan et al., 2009; Feng et al., 2010a; Feng et al., 2010b; Feng et al., 2010c; Pan et al., 2010a; Pan et al., 2010b; Xu et al., 2010; Chen and Li, 2011; Hwang et al., 2014; Liu, 2016) were produced by Horizontal treadmill exercise, 10 models (Li et al., 2009; Gao et al., 2011; Xu et al., 2011; Zhao et al., 2014; Liu et al., 2015; Oh et al., 2015; Wang et al., 2015; Shi et al., 2016; Yao, 2016; Ma et al., 2017) by weight-loaded swimming test (WLST), 6 models (Song et al., 2013; Qi et al., 2014; Wang et al., 2014; Bao et al., 2016; Zheng et al., 2017; Delgado et al., 2019) by Forced swimming test (FST) and 3models (Choi et al., 2011; Oh et al., 2015; Shi et al., 2016) by Rota-rod test. The non-functional liquid, normal saline or no treatment control was introduced in all 30 studies. Thirteen studies (Li et al., 2009 Xu et al., 2011; Song et al., 2013; Qi et al., 2014; Zhao et al., 2014; Liu et al., 2015; Oh et al., 2015; Wang et al., 2015; Bao et al., 2016; Shi et al., 2016; Yao, 2016; Ma et al., 2017; Delgado et al., 2019) utilized swimming time as outcome measure, and climbing time in 1 study (Shi et al., 2016). Serum biochemical parameters, including BLA, BUN, glucose (GLU), 
A
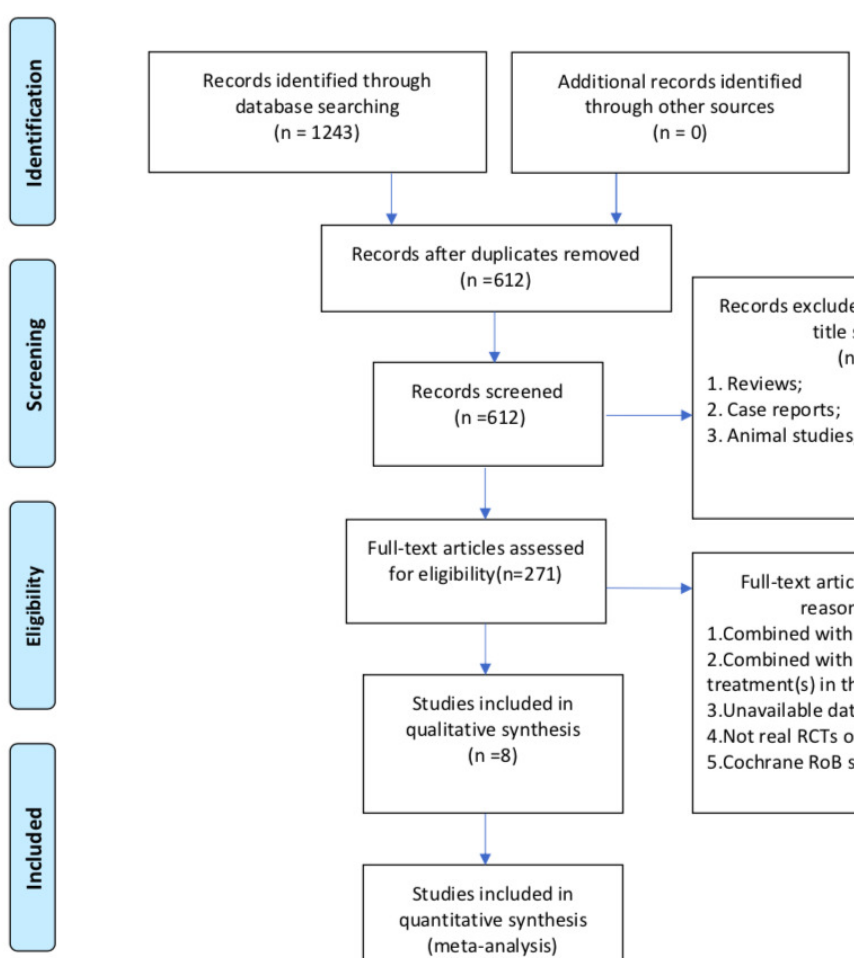

Records after duplicates removed

( $n=612)$

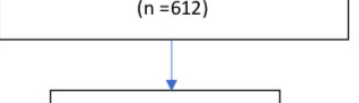

Records excluded after abstract and title screening

1. Reviews;

$(n=341)$

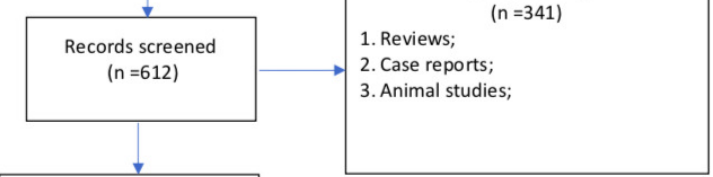

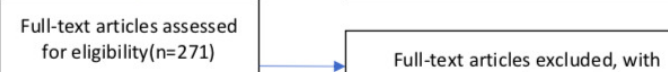
reasons $(n=263)$ 1.Combined with other diseases; 2.Combined with other herbal
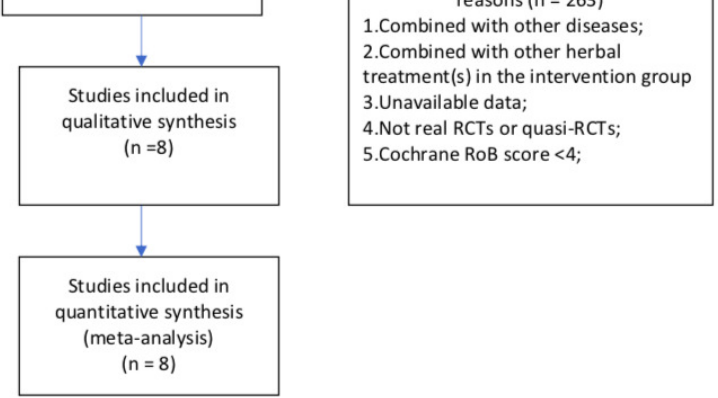

3.Unavailable data;

4.Not real RCTs or quasi-RCT
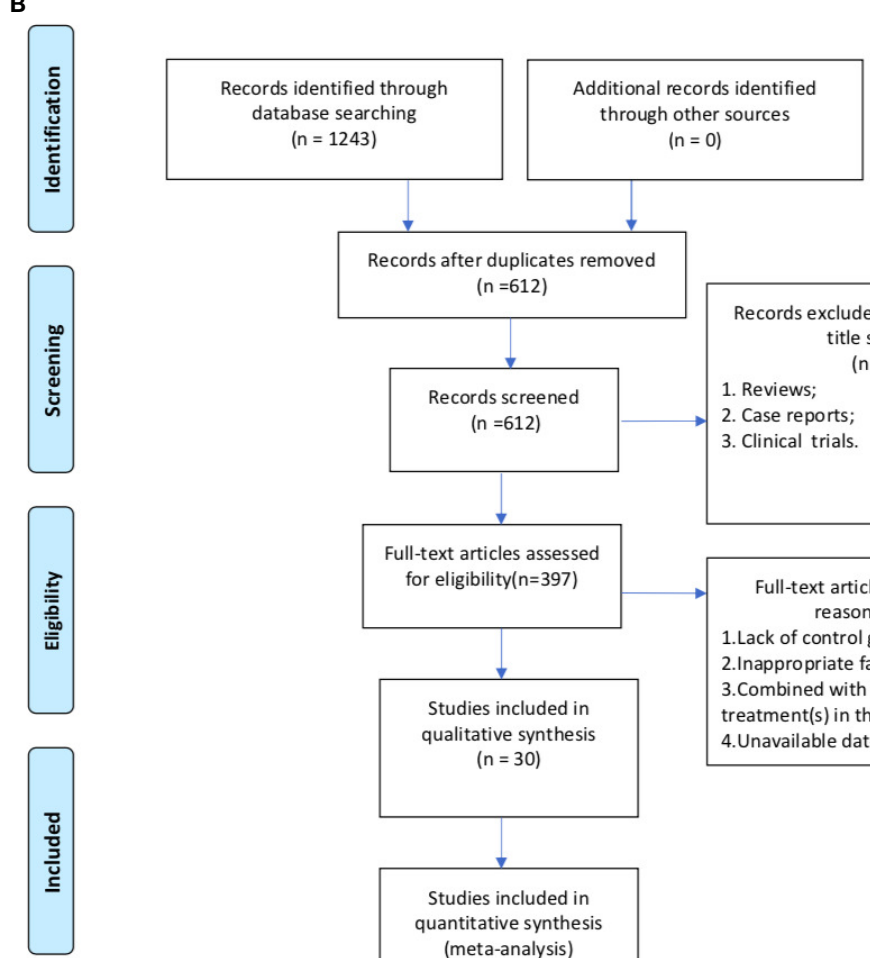

Records after duplicates removed $(n=612)$

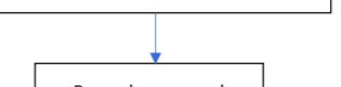

Records excluded after abstract and title screening

1. Reviews;

$(n=215)$
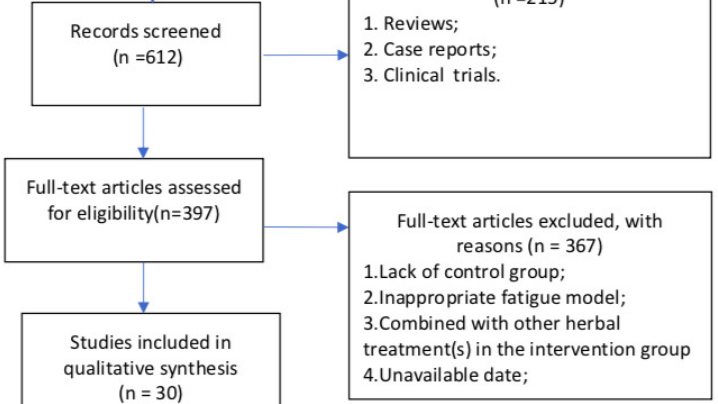

qualitative synthesis

$n=30)$

4.Unavailable date

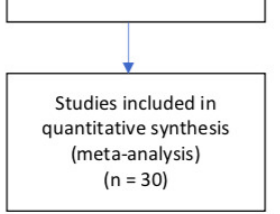

FIGURE 1 | (A) Flow diagram of randomized control trials selection process. (B) Flow diagram of animal experiments selection process. 
TABLE 1 | Characteristics of the included randomized control trials.

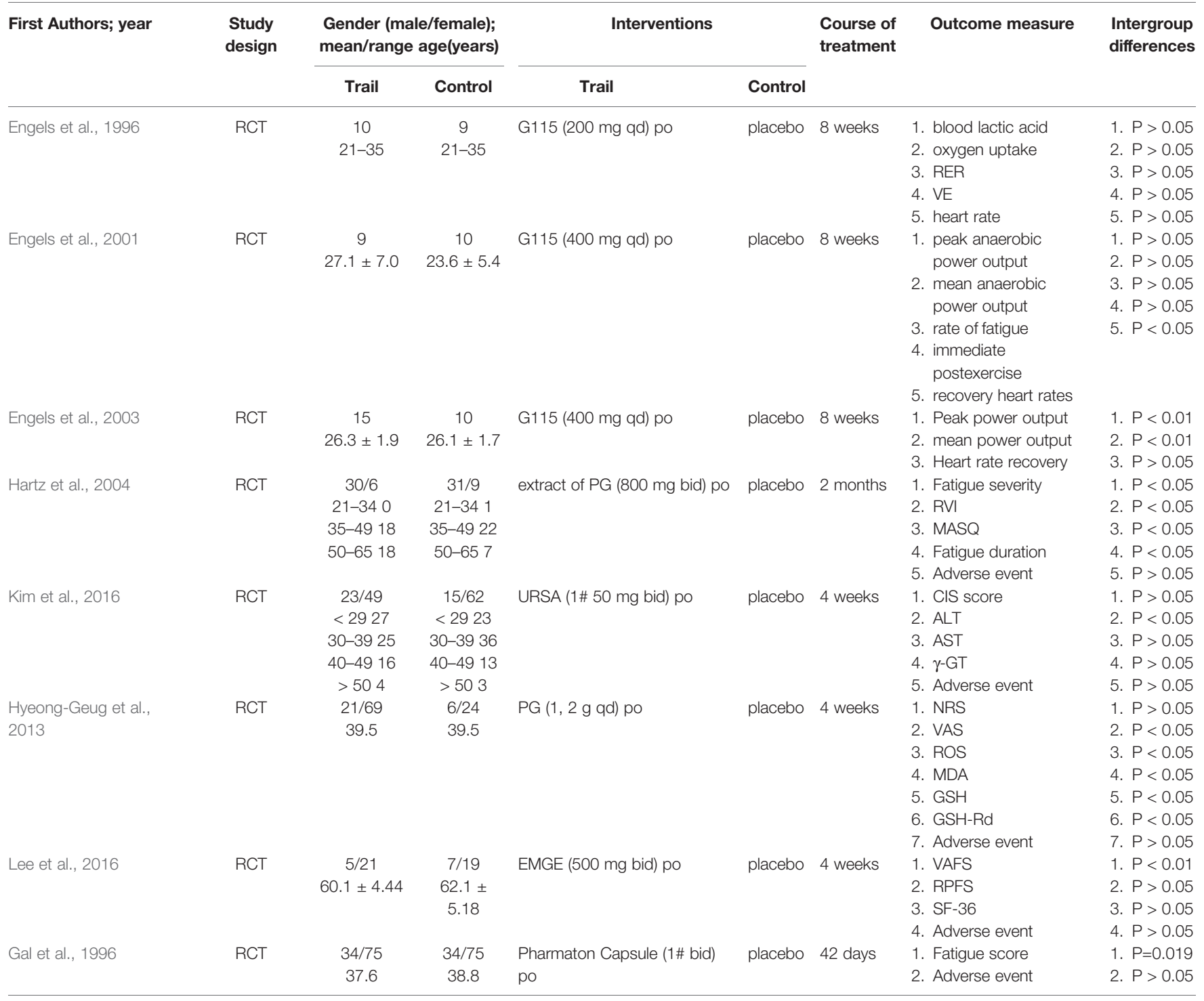

ALT, alanine aminotransferase; AST, aspartate aminotransferase; CIS, checklist individual strength; GSH, glutathione; GSH-Rd, glutathione reductase; G115, standardized P. ginseng C.A. Mey concentrate;MASQ, Mood and Anxiety Symptom Questionnaire; MDA, malondialdehyde; NRS, self-rating numeric scale; PG, P. ginseng C.A. Mey; RER, respiratory exchange ratio; ROS, reactive oxygen species; RPFS, Revised Piper Fatigue Scale; RVI, Rand Vitality Index; SF-36, Short-Form Health Survey; VAS, visual analog scale; VAFS, visual analog fatigue scale; VE, minute ventilation.

creatine kinase (CK), MDA, superoxide dismutase (SOD), glutathione peroxidase (GSH-Px), and $\mathrm{LDH}$ were reported in 21 studies (Feng et al., 2009c; Li et al., 2009; Pan et al., 2010a; Choi et al., 2011; Gao et al., 2011; Xu et al., 2011; Song et al., 2013; Hwang et al., 2014; Qi et al., 2014; Wang et al., 2014; Zhao et al., 2014; Liu et al., 2015; Oh et al., 2015; Wang et al., 2015; Bao et al., 2016; Liu, 2016; Shi et al., 2016; Yao, 2016; Ma et al., 2017; Zheng et al., 2017; Delgado et al., 2019); skeletal muscle biochemical parameters, including glycogen, MDA, SOD, catalase (CAT), and GSH-Px in 13 studies (Li et al., 2009; Feng et al., 2010a; Feng et al., 2010b; Feng et al., 2010c; Pan et al., 2010b; Xu et al., 2010; Gao et al., 2011; Zhao et al., 2014; Wang et al., 2015; Bao et al., 2016; Liu, 2016; Ma et al., 2017; Delgado et al., 2019); liver biochemical parameters, including glycogen, MDA, SOD, and GSH-Px in 14 studies (Feng et al., 2009c; Pan et al., 2010a; Gao et al., 2011; Xu et al., 2011; Hwang et al., 2014; Qi et al., 2014; Zhao et al., 2014; Liu et al., 2015; Wang et al., 2015; Bao et al., 2016; Shi et al., 2016; Yao, 2016; Ma et al., 2017; Delgado et al., 2019); brain tissue biochemical parameters, including acetylcholine (Ach), dopamine (DA), gamma-aminobutyric acid (GABA), 5-hydroxytryptamine (5-HT) in 5 study (Feng et al., 2009a; Feng et al., 2009b; Feng et al., 2009c; Chen and Li, 2011; Liu, 2016); and body weight in 7 studies (Li et al., 2009; Xu et al., 2011; Song et al., 2013; Hwang et al., 2014; Bao et al., 2016; Liu, 2016; Shi et al., 2016). Approach to achieving of PGC was reported in 15 studies (Feng et al., 2009a; Feng et al., 2009b; Feng et al., 2009c; Li et al., 2009; Pan et al., 2009; Feng et al., 2010a; Feng et al., 2010b; Feng et al., 2010c; Pan et al., 2010a; Pan et al., 2010b; Xu et al., 2010; Chen and Li, 2011; Choi et al., 2011; Song et al., 2013; 
TABLE 2 | Statement of the characteristic of Panax ginseng C. A. Mey and its compounds(a).

\begin{tabular}{|c|c|c|c|c|}
\hline $\begin{array}{l}\text { First Authors; } \\
\text { year }\end{array}$ & Drugs; & Approach to achieving & $\begin{array}{l}\text { Chemical } \\
\text { analysis }\end{array}$ & Composition \\
\hline Engels et al., 1996 & G115 & $\begin{array}{l}\text { Pharmaten Ltd., Lugano, } \\
\text { Switzerland }\end{array}$ & included-HPLC & $\begin{array}{l}\text { Contain } 4 \% \text { ginsenosides(Rgl } 0.548 \% \text {, Re } 0.352 \% \text {, Rf } 0.270 \% \text {, Rg2 } \\
0.065 \% \text {, Rbl 1.338\%, Rc 0.714\%, Rb2 0.567\%, Rd 0.286\%) HPLC data } \\
\text { from F. Soldati and Sticher, } 1980 .\end{array}$ \\
\hline Engels et al., 2001 & G115 & $\begin{array}{l}\text { Pharmaten Ltd., Lugano, } \\
\text { Switzerland }\end{array}$ & included-HPLC & $\begin{array}{l}\text { Contain } 4 \% \text { ginsenosides(Rgl 0.548\%, Re 0.352\%, Rf 0.270\%, Rg2 } \\
0.065 \% \text {, Rbl 1.338\%, Rc 0.714\%, Rb2 0.567\%, Rd 0.286\%) HPLC data } \\
\text { from F. Soldati and Sticher, } 1980 .\end{array}$ \\
\hline Engels et al., 2003 & G115 & $\begin{array}{l}\text { Pharmaten Ltd., Lugano, } \\
\text { Switzerland }\end{array}$ & included-HPLC & $\begin{array}{l}\text { Contain } 4 \% \text { ginsenosides(Rgl } 0.548 \% \text {, Re } 0.352 \% \text {, Rf } 0.270 \% \text {, Rg2 } \\
0.065 \% \text {, Rbl 1.338\%, Rc 0.714\%, Rb2 0.567\%, Rd 0.286\%) HPLC data } \\
\text { from F. Soldati and Sticher, } 1980 .\end{array}$ \\
\hline Hartz et al., 2004 & extract ofPG & $\begin{array}{l}\text { Frontier Herbs of Norway, } \\
\text { IA., Norway }\end{array}$ & included-HPLC & Contain $0.112 \%$ eleutheroside B and eleutheroside $\mathrm{E}$ \\
\hline Kim et al., 2016 & $\begin{array}{l}\text { URSA Complex } \\
\text { (contain dried PG } \\
\text { extract); }\end{array}$ & not reported & included-HPLC & Contain dried ginseng extracts $50 \mathrm{mg}$ \\
\hline $\begin{array}{l}\text { Hyeong-Geug } \\
\text { et al., } 2013\end{array}$ & $\begin{array}{l}\text { bespoke } 20 \% \text { ethanol } \\
\text { extract of PG; }\end{array}$ & $\begin{array}{l}\text { Guryoung Pharmaceutical } \\
\text { Company, Ltd., Cheorwon, } \\
\text { South Korea }\end{array}$ & included-HPLC & $\begin{array}{l}\text { Contain } 2 \% \text { ginsenosides (Rb3 0.633\%, Rb1 0.514\%, Rb2 0.36\%, Rc } \\
0.261 \% \text {, Rg3 0.108\%, Rd 0.043\%, Rh2 0.0002\%) HPLC data from } \\
\text { Hyeong-Geug et al., } 2013 .\end{array}$ \\
\hline Lee et al., 2016 & $\begin{array}{l}\text { Enzyme-modified } \\
\text { ginseng extract } \\
\text { (EMGE); }\end{array}$ & not reported & included-HPLC & $\begin{array}{l}\text { Rg1, Rh1, Rb1, Rg3(S), Rg3(R), Compound K, Rh2(S), Rh2(R);(similar to } \\
\text { G115 by HPLC) HPLC data from Lee et al., } 2016 .\end{array}$ \\
\hline Gal et al., 1996 & $\begin{array}{l}\text { Pharmaton Capsule } \\
\text { (contain G115); }\end{array}$ & $\begin{array}{l}\text { Pharmaten Ltd., Lugano, } \\
\text { Switzerland }\end{array}$ & included-HPLC & $\begin{array}{l}\text { Contain } 4 \% \text { ginsenosides (Rgl 0.548\%, Re 0.352\%, Rf 0.270\%, Rg2 } \\
0.065 \% \text {, Rbl 1.338\%, Rc 0.714\%, Rb2 0.567\%, Rd 0.286\%) HPLC data } \\
\text { from F. Soldati and Sticher, } 1980 .\end{array}$ \\
\hline
\end{tabular}

G115, standardized P. ginseng C.A. Mey concentrate; HPLC: high-performance liquid chromatography; PG, Panaxginseng C.A. Mey.

Hwang et al., 2014; Wang et al., 2014; Liu et al., 2015; Oh et al., 2015; Wang et al., 2015; Bao et al., 2016; Liu, 2016; Shi et al., 2016; Zheng et al., 2017; Delgado et al., 2019) and quality control of PGC was reported in 8 studies (Feng et al., 2009c; Feng et al., 2010c; Chen and Li, 2011; Wang et al., 2014; Liu et al., 2015; Oh et al., 2015; Zheng et al., 2017; Delgado et al., 2019). Tables 3 and 4 show the characteristics of included publications.

\section{Study Quality Randomized Clinical Trials}

Table 5 illustrates the methodological quality of 8 RCTs based on the Cochrane Collaboration's tool. All of them were ranged from 4 to 7 points. All included studies reported the method of random sequences generation, the criteria of a double-blind study design, and taking the complete outcome data into account. Three studies (Hartz et al., 2004; Hyeong-Geug et al., 2013; Lee et al., 2016) reported using allocation concealment. Two studies (Hartz et al., 2004; Lee et al., 2016) applied blinding specifically during outcome measure assessment. The protocols of 3 studies (Hyeong-Geug et al., 2013; Kim et al., 2016; Lee et al., 2016) were registered in the Clinical Trial Registry. In other bias, all eight studies were supported by nonprofit institutions and accounted for baseline comparability, but no study provided sample size estimation information.

\section{Animal Studies}

The quality scores of studies included varied from 4 to 7 out of 10 points with the average of 5.73. Of which, 4 studies (Feng et al., 2009b; Wang et al., 2015; Liu, 2016; Yao, 2016) got 4 points; 6 studies (Li et al., 2009; Xu et al., 2010; Chen and Li, 2011; Xu et al., 2011; Liu et al., 2015; Shi et al., 2016) got 5 points; 13 studies (Feng et al., 2009a; Feng et al., 2009b; Feng et al., 2010a; Feng et al., 2010b; Feng et al., 2010c; Pan et al., 2010a; Pan et al., 2010b; Choi et al., 2011; Gao et al., 2011; Song et al., 2013; Wang et al., 2014; Zhao et al., 2014; Zheng et al., 2017) got 6 points; and 6 studies (Hwang et al., 2014; Qi et al., 2014; Oh et al., 2015; Bao et al., 2016; Ma et al., 2017; Delgado et al., 2019) got 7 points. All the included records described appropriate animal models (aged or female involved) and used an anesthetic without significant intrinsic neuroprotective activity. However, no study reported blinded induction of model, blinding their assessment of outcome, and a sample size calculation. Twenty-nine studies (Feng et al., 2009a; Feng et al., 2009b; Feng et al., 2009c; Li et al., 2009; Pan et al., 2009; Feng et al., 2010a; Feng et al., 2010b; Feng et al., 2010c; Pan et al., 2010a; Pan et al., 2010b; Xu et al., 2010; Chen and Li, 2011; Choi et al., 2011; Gao et al., 2011; Xu et al., 2011; Song et al., 2013; Hwang et al., 2014; Qi et al., 2014; Wang et al., 2014; Zhao et al., 2014; Liu et al., 2015; Oh et al., 2015; Wang et al., 2015; Bao et al., 2016; Shi et al., 2016; Yao, 2016; Ma et al., 2017; Zheng et al., 2017; Delgado et al., 2019) were peerreviewed publications. Twenty-seven studies (Feng et al., 2009a; Feng et al., 2009b; Feng et al., 2009c; Li et al., 2009; Pan et al., 2009; Feng et al., 2010a; Feng et al., 2010b; Feng et al., 2010c; Pan et al., 2010a; Pan et al., 2010b; Xu et al., 2010; Chen and Li, 2011; Gao et al., 2011; Xu et al., 2011; Song et al., 2013; Hwang et al., 2014; Qi et al., 2014; Zhao et al., 2014; Liu et al., 2015; Oh et al., 2015; Wang et al., 2015; Bao et al., 2016; Liu, 2016; Shi et al., 2016; Yao, 2016; Ma et al., 2017; Delgado et al., 2019) allocated randomly to treatment group and control group. Twenty-four studies (Feng et al., 2009a; Feng et al., 2009b; Pan 
TABLE 3 | Characteristics of the included animal studies

\begin{tabular}{|c|c|c|c|c|c|c|c|}
\hline Study (years) & $\begin{array}{l}\text { Species } \\
\text { Sex } \\
\text { Weight } \\
\text { N }\end{array}$ & Anesthetic & Model (method) & $\begin{array}{l}\text { Experimental group (drugs, } \\
\text { concentration, administration, } \\
\text { duration) }\end{array}$ & Control group & Outcome measure & $\begin{array}{l}\text { Intergroup } \\
\text { differences* }\end{array}$ \\
\hline Xu et al., 2011 & $\begin{array}{l}\text { KM mice } \\
\text { NG } \\
18-22 \mathrm{~g} \\
240\end{array}$ & - & weight-loaded swimming (WLS) test & $\begin{array}{l}\text { ginseng protein }(0.1,0.2,0.4 \mathrm{~g} / \mathrm{kg}) \\
\text { ig, } 30 \text { days }\end{array}$ & $\begin{array}{l}\text { Distilled water for } \\
\text { same volume }\end{array}$ & $\begin{array}{l}\text { 1. Mice body weight } \\
\text { 2. Swimming time in } \\
\text { mice } \\
\text { 3. BLA } \\
\text { 4. Liver glycogen } \\
\text { 5. Serum urea nitrogen }\end{array}$ & $\begin{array}{l}\text { 1. } P>0.05 \\
\text { 2. } P>0.05 \\
\text { 3. } P<0.05 \\
\text { 4. } P>0.05 \\
\text { 5. } P>0.05\end{array}$ \\
\hline Wang et al., 2010 & $\begin{array}{l}\text { ICR mice } \\
\text { M } \\
(11-12 \text { weeks }) \\
104\end{array}$ & - & Forced swimming test & $\begin{array}{l}\text { WGP }(50,100,200 \mathrm{mg} / \mathrm{kg}) \\
W G P A(40,100,160,200 \mathrm{mg} / \mathrm{kg}) \\
W G P N(40,100,160,200 \mathrm{mg} / \mathrm{kg}) \mathrm{ig} \text {, } \\
15 \text { days }\end{array}$ & $\begin{array}{l}\text { NS for same } \\
\text { volume }\end{array}$ & $\begin{array}{l}\text { 1. Serum GLU } \\
\text { 2. Serum TG } \\
\text { 3. Serum CK } \\
\text { 4. Serum LDH } \\
\text { 5. Serum MDA } \\
\text { 6. Serum SOD } \\
\text { 7. Serum GSH-Px }\end{array}$ & $\begin{array}{l}\text { 1. } P<0.01 \\
\text { 2. } P<0.01 \\
\text { 3. } P<0.05 \\
\text { 4. } P<0.05 \\
\text { 5. } P<0.001 \\
\text { 6. } P<0.05 \\
\text { 7. } P<0.05\end{array}$ \\
\hline Wang et al., 2013 & $\begin{array}{l}\text { ICR mice } \\
\mathrm{M} \\
(11-12 \text { weeks }) \\
104\end{array}$ & - & Forced swimming test & $\begin{array}{l}\text { WGPA }(200 \mathrm{mg} / \mathrm{kg}) \\
\text { WGPA-A }(200 \mathrm{mg} / \mathrm{kg}) \\
\text { WGPA-N(200 mg/kg) ig, } 15 \text { days }\end{array}$ & $\begin{array}{l}\text { NS for same } \\
\text { volume }\end{array}$ & $\begin{array}{l}\text { 1. Serum MDA } \\
\text { 2. Serum SOD } \\
\text { 3. SerumGSH-Px } \\
\text { 4. Serum LDH }\end{array}$ & $\begin{array}{l}\text { 1. } P<0.001 \\
\text { 2. } P<0.01 \\
\text { 3. } P<0.01 \\
\text { 4. } P<0.05\end{array}$ \\
\hline Song et al., 2013 & $\begin{array}{l}\text { Wistar rat } \\
\mathrm{M} \\
180-220 \mathrm{~g} \\
60\end{array}$ & - & Forced swimming test & PTS $(25,50,100 \mathrm{mg} / \mathrm{kg})$ ig, 7 weeks & $\begin{array}{l}\text { NS for same } \\
\text { volume }\end{array}$ & $\begin{array}{l}\text { 1. Mice body weight } \\
\text { 2. Swimming time in } \\
\text { mice } \\
\text { 3. Serum GLU } \\
\text { 4. Serum urea nitrogen } \\
\text { 5. Blood lactic acid }\end{array}$ & $\begin{array}{l}\text { 1. } P>0.05 \\
\text { 2. } P<0.05 \\
\text { 3. } P<0.05 \\
\text { 4. } P<0.05 \\
\text { 5. } P<0.05\end{array}$ \\
\hline Xu et al., 2010 & $\begin{array}{l}\text { SD rat } \\
\mathrm{M} \\
180-200 \mathrm{~g} \\
30\end{array}$ & - & Horizontal treadmill exercise & $\begin{array}{l}\text { Ginsenoside Rb1 }(50 \text { mg/kg) ig, } 14 \\
\text { days }\end{array}$ & $\begin{array}{l}\text { NS for same } \\
\text { volume }\end{array}$ & $\begin{array}{l}\text { 1. Skeletal muscle MDA } \\
\text { 2. Skeletal muscle SOD }\end{array}$ & $\begin{array}{l}\text { 1. } P<0.01 \\
\text { 2. } P<0.05\end{array}$ \\
\hline Feng et al., 2010a & $\begin{array}{l}\text { SD rat } \\
\mathrm{M} \\
180-220 \mathrm{~g} \\
40\end{array}$ & - & Horizontal treadmill exercise & $\begin{array}{l}\text { Ginsenoside }(50 \mathrm{mg} / \mathrm{kg}) \\
\text { Ginsenoside Rb1 }(50 \mathrm{mg} / \mathrm{kg}) \mathrm{ig}, 14 \\
\text { days }\end{array}$ & $\begin{array}{l}\text { NS for same } \\
\text { volume }\end{array}$ & $\begin{array}{l}\text { 1. Calcium content in } \\
\text { skeletal muscle cell } \\
\text { 2. Mitochondrial } \\
\text { membrane potential } \\
\text { 3. Skeletal muscle MDA } \\
\text { 4. Skeletal muscle SOD } \\
\text { 5. Skeletal muscle GLU }\end{array}$ & $\begin{array}{l}\text { 1. } P<0.05 \\
\text { 2. } P<0.05 \\
\text { 3. } P<0.05 \\
\text { 4. } P<0.05 \\
\text { 5. } P<0.05\end{array}$ \\
\hline Feng et al., 2009a & $\begin{array}{l}\text { SD rat } \\
M \\
180-220 \mathrm{~g} \\
40\end{array}$ & - & Horizontal treadmill exercise & $\begin{array}{l}\text { Ginsenoside }(50 \mathrm{mg} / \mathrm{kg}) \\
\text { Ginsenoside Re }(50 \mathrm{mg} / \mathrm{kg}) \mathrm{ig}, 14 \\
\text { days }\end{array}$ & $\begin{array}{l}\text { NS for same } \\
\text { volume }\end{array}$ & $\begin{array}{l}\text { 1. Hypothalamus Ach } \\
\text { 2. Hypothalamus DA } \\
\text { 3. Hypothalamus 5-HT } \\
\text { 4. Hypothalamus GABA }\end{array}$ & $\begin{array}{l}\text { 1. } P<0.01 \\
\text { 2. } P<0.01 \text { or } \\
P<0.05 \\
\text { 3. } P<0.01 \\
\text { 4. } P<0.01\end{array}$ \\
\hline Liu, 2016 & $\begin{array}{l}\text { SD rat } \\
M \\
240 \pm 20 \mathrm{~g} \\
40\end{array}$ & - & Horizontal treadmill exercise & $\begin{array}{l}\text { Ginsenoside } \mathrm{Rg} 1(30 \mathrm{mg} / \mathrm{kg}) \mathrm{ig}, 8 \\
\text { weeks }\end{array}$ & $\begin{array}{l}\text { NS for same } \\
\text { volume }\end{array}$ & $\begin{array}{l}\text { 1. Mice body weight } \\
\text { 2. Exhaustion time } \\
\text { 3. Skeletal muscle } \\
\text { SOD } \\
\text { 4. Skeletal muscle } \\
\text { GSH-Px }\end{array}$ & $\begin{array}{l}\text { 1. } P>0.05 \\
\text { 2. } P<0.01 \\
\text { 3. } P<0.01 \\
\text { 4. } P<0.05 \\
\text { 5. } P<0.05 \\
\text { 6. } P<0.05\end{array}$ \\
\hline
\end{tabular}




\begin{tabular}{|c|c|c|c|c|c|c|c|}
\hline Study (years) & $\begin{array}{l}\text { Species } \\
\text { Sex } \\
\text { Weight } \\
\text { N }\end{array}$ & Anesthetic & Model (method) & $\begin{array}{l}\text { Experimental group (drugs, } \\
\text { concentration, administration, } \\
\text { duration) }\end{array}$ & Control group & Outcome measure & $\begin{array}{l}\text { Intergroup } \\
\text { differences }\end{array}$ \\
\hline & & & & & & $\begin{array}{l}\text { 5. Skeletal muscle } \\
\text { CAT } \\
\text { 6. Skeletal muscle } \\
\text { MDA } \\
\text { 7. Skeletal muscle } \\
\text { carbonylation } \\
\text { protein content } \\
\text { 8. Brain tissue SOD } \\
\text { 9. Brain tissue GSH- } \\
\text { Px } \\
\text { 10. Brain tissue CAT } \\
\text { 11. Brain tissue MDA } \\
\text { 12. Brain tissue } \\
\text { carbonylation } \\
\text { protein content } \\
\text { 13. Blood lactic acid }\end{array}$ & $\begin{array}{l}\text { 7. } P<0.05 \\
\text { 8. } P<0.01 \\
\text { 9. } P<0.05 \\
\text { 10. } P<0.05 \\
\text { 11. } P>0.05 \\
\text { 12. } P<0.05 \\
\text { 13. } 13 . \\
\quad P>0.05\end{array}$ \\
\hline Feng et al., 2010b & $\begin{array}{l}\text { SD rat } \\
\mathrm{M} \\
180-220 \mathrm{~g} \\
40\end{array}$ & - & Horizontal treadmill exercise & $\begin{array}{l}\text { Ginsenoside Rb1(50 mg/kg) } \\
\text { Ginsenoside Rg1(50 mg/kg) ig, } 14 \\
\text { days }\end{array}$ & $\begin{array}{l}\text { NS for same } \\
\text { volume }\end{array}$ & $\begin{array}{l}\text { 1. Hypothalamus Ach } \\
\text { 2. Hypothalamus DA } \\
\text { 3. Hypothalamus 5-HT } \\
\text { 4. Hypothalamus GABA }\end{array}$ & $\begin{array}{l}\text { 1. } P<0.01 \\
\text { 2. } P<0.01 \\
\text { 3. } P<0.01 \\
\text { 4. } P<0.01\end{array}$ \\
\hline Wang et al., 2015 & $\begin{array}{l}\mathrm{KM} \text { mice } \\
\mathrm{M} \\
20 \pm 2 \mathrm{~g} \\
180\end{array}$ & - & weight-loaded swimming (WLS) test & $\begin{array}{l}\text { Ginsenoside } \operatorname{Rg} 1(5,10,20,40,80 \\
\mathrm{mg} / \mathrm{kg}) \mathrm{ig}, 2 \text { weeks }\end{array}$ & $\begin{array}{l}\text { NS for same } \\
\text { volume }\end{array}$ & $\begin{array}{l}\text { 1. Swimming time } \\
\text { 2. Blood lactic acid } \\
\text { 3. Serum BUN } \\
\text { 4. Serum GLU } \\
\text { 5. Muscle glycogen } \\
\text { 6. Hepatic glycogen }\end{array}$ & $\begin{array}{l}\text { 1. } P>0.05 \\
\text { 2. } P>0.05 \\
\text { 3. } P>0.05 \\
\text { 4. } P>0.05 \\
\text { 5. } P<0.01 \\
\text { 6. } P<0.05\end{array}$ \\
\hline Yao, 2016 & $\begin{array}{l}\text { mice } \\
\mathrm{M} \\
19-22 \mathrm{~g} \\
80\end{array}$ & - & $x^{2}$ & $\begin{array}{l}\text { Ginsenoside }(0.8,1.6,3.2 \mathrm{~g} / \mathrm{kg}) \text { ig, } \\
28 \text { days }\end{array}$ & $\begin{array}{l}\text { distilled water for } \\
\text { same volume }\end{array}$ & $\begin{array}{l}\text { 1. Serum BUN } \\
\text { 2. Serum LDH } \\
\text { 3. Serum BLA } \\
\text { 4. Serum CK } \\
\text { 5. Hepatic glycogen } \\
\text { 6. Liver MDA } \\
\text { 7. Liver SOD } \\
\text { 8. Liver GSH-Px } \\
\text { 9. Swimming time }\end{array}$ & $\begin{array}{l}\text { 1. } P<0.05 \\
\text { 2. } P<0.05 \\
\text { 3. } P<0.05 \\
\text { 4. } P<0.05 \\
\text { 5. } P<0.05 \\
\text { 6. } P<0.05 \\
\text { 7. } P<0.05 \\
\text { 8. } P<0.05 \\
\text { 9. } P<0.05\end{array}$ \\
\hline Liu et al., 2015 & $\begin{array}{l}\text { mice } \\
\mathrm{M} \\
18-22 \mathrm{~g} \\
40\end{array}$ & - & weight-loaded swimming (WLS) test & $\begin{array}{l}\text { Ginsenoside }(0.8,1.6,3.2 \mathrm{~g} / \mathrm{kg}) \mathrm{ig} \text {, } \\
28 \text { days }\end{array}$ & $\begin{array}{l}\text { distilled water for } \\
\text { same volume }\end{array}$ & $\begin{array}{l}\text { 1. Serum BUN } \\
\text { 2. Serum LDH } \\
\text { 3. Serum BLA } \\
\text { 4. Serum CK } \\
\text { 5. Hepatic glycogen } \\
\text { 6. Liver MDA } \\
\text { 7. Liver SOD } \\
\text { 8. Liver GSH-Px } \\
\text { 9. Swimming time }\end{array}$ & $\begin{array}{l}\text { 1. } P<0.001 \\
\text { 2. } P<0.01 \\
\text { 3. } P<0.05 \\
\text { 4. } P<0.001 \\
\text { 5. } P<0.01 \\
\text { 6. } P<0.01 \\
\text { 7. } P>0.05 \\
\text { 8. } P>0.05 \\
\text { 9. } P<0.01\end{array}$ \\
\hline
\end{tabular}




\begin{tabular}{|c|c|c|c|c|c|c|c|}
\hline Study (years) & $\begin{array}{c}\text { Species } \\
\text { Sex } \\
\text { Weight } \\
\text { N }\end{array}$ & Anesthetic & Model (method) & $\begin{array}{l}\text { Experimental group (drugs, } \\
\text { concentration, administration, } \\
\text { duration) }\end{array}$ & Control group & Outcome measure & $\begin{array}{l}\text { Intergroup } \\
\text { differences* }\end{array}$ \\
\hline Feng et al., 2009b & $\begin{array}{l}\text { SD rat } \\
\mathrm{M} \\
180-220 \mathrm{~g} \\
30\end{array}$ & - & Horizontal treadmill exercise & $\begin{array}{l}\text { Ginsenoside } \operatorname{Re}(50 \text { mg/kg) ig, } 14 \\
\text { days }\end{array}$ & $\begin{array}{l}\text { NS for same } \\
\text { volume }\end{array}$ & $\begin{array}{l}\text { 1. 1.Serum MDA } \\
\text { 2. Liver MDA } \\
\text { 3. Skeletal muscle MDA } \\
\text { 4. Serum SOD } \\
\text { 5. Liver SOD } \\
\text { 6. Skeletal muscle SOD }\end{array}$ & $\begin{array}{l}\text { 1. } P<0.01 \\
\text { 2. } P<0.01 \\
\text { 3. } P<0.01 \\
\text { 4. } P<0.01 \\
\text { 5. } P<0.01 \\
\text { 6. } P<0.01\end{array}$ \\
\hline Feng et al., 2010c & $\begin{array}{l}\text { SD rat } \\
\mathrm{M} \\
180-220 \mathrm{~g} \\
30\end{array}$ & - & Horizontal treadmill exercise & $\begin{array}{l}\text { Ginsenoside Rg1 } 150 \text { mg/kg) ig, } 14 \\
\text { days }\end{array}$ & $\begin{array}{l}\text { NS for same } \\
\text { volume }\end{array}$ & $\begin{array}{l}\text { 1. Calcium content in } \\
\text { skeletal muscle cell } \\
\text { 2. Mitochondrial } \\
\text { membrane potential } \\
\text { 3. Skeletal muscle SOD } \\
\text { 4. Skeletal muscle MDA }\end{array}$ & $\begin{array}{l}\text { 1. } P<0.01 \\
\text { 2. } P<0.01 \\
\text { 3. } P<0.01 \\
\text { 4. } P<0.01\end{array}$ \\
\hline Pan et al., 2010a & $\begin{array}{l}\text { SD rat } \\
\mathrm{M} \\
180-220 \mathrm{~g} \\
30\end{array}$ & 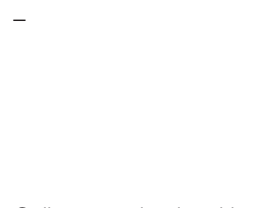 & Horizontal treadmill exercise & Ginsenosides (50 mg/kg) ig, 14 days & $\begin{array}{l}\text { NS for same } \\
\text { volume }\end{array}$ & $\begin{array}{l}\text { 1. Calcium content in } \\
\text { skeletal muscle cell } \\
\text { 2. Mitochondrial } \\
\text { membrane potential } \\
\text { 3. Skeletal muscle SOD } \\
\text { 4. Skeletal muscle MDA }\end{array}$ & $\begin{array}{l}\text { 1. } P<0.01 \\
\text { 2. } P<0.01 \\
\text { 3. } P<0.01 \\
\text { 4. } P<0.01\end{array}$ \\
\hline Chen and Li, 2011 & $\begin{array}{l}\text { SD rat } \\
M \\
160-200 \mathrm{~g} \\
45\end{array}$ & $\begin{array}{l}\text { Celiac anesthesia with } \\
\text { chloral hydrate }\end{array}$ & Horizontal treadmill exercise & Ginsenosides (70 mg/kg) ig, 8 weeks & $\begin{array}{l}\text { NS for same } \\
\text { volume }\end{array}$ & $\begin{array}{l}\text { 1. Hippocampus tissue } \\
\text { GABA } \\
\text { 2. Hippocampus tissue } \\
\text { Ach } \\
\text { 3. Hippocampus tissue } \\
\mathrm{NE} \\
\text { 4. Hippocampus tissue } \\
\text { DA } \\
\text { 5. Hippocampus tissue } \\
\text { 5-HT } \\
\text { 6. DA/5-HT }\end{array}$ & $\begin{array}{l}\text { 1. } P<0.05 \\
\text { 2. } P<0.05 \\
\text { 3. } P<0.05 \\
\text { 4. } P<0.05 \\
\text { 5. } P<0.05 \\
\text { 6. } P<0.05\end{array}$ \\
\hline Pan et al., 2009 & $\begin{array}{l}\text { SD rat } \\
M \\
180-220 \mathrm{~g} \\
40\end{array}$ & - & Horizontal treadmill exercise & $\begin{array}{l}\text { Ginsenosides }(50 \text { mg/kg) } \\
\text { Ginsenoside Rg1 }(50 \text { mg/kg) ig, } 14 \\
\text { days }\end{array}$ & $\begin{array}{l}\text { NS for same } \\
\text { volume }\end{array}$ & $\begin{array}{l}\text { 1. Hippocampus tissue } \\
\text { GABA } \\
\text { 2. Hippocampus tissue } \\
\text { DA } \\
\text { 3. Hippocampus tissue } \\
\text { 5-HT } \\
\text { 4. Hippocampus tissue } \\
\text { Ach }\end{array}$ & $\begin{array}{l}\text { 1. } P<0.01 \\
\text { 2. } P<0.05 \\
\text { 3. } P<0.01 \\
\text { 4. } P<0.05\end{array}$ \\
\hline Li et al., 2009 & $\begin{array}{l}\text { KM mice } \\
\mathrm{M} \\
18-22 \mathrm{~g} \\
80\end{array}$ & - & weight-loaded swimming (WLS) test & $\begin{array}{l}\text { Pseudo- ginsenoside } G Q(P G Q)(0.2 \text {, } \\
0.4,0.8 \text { g/kg) ig, } 5 \text { weeks }\end{array}$ & $\begin{array}{l}\text { NS for same } \\
\text { volume }\end{array}$ & $\begin{array}{l}\text { 1. Mice body weight } \\
\text { 2. Swimming time in } \\
\text { mice } \\
\text { 3. Blood lactic acid } \\
\text { 4. Serum BUN } \\
\text { 5. Serum LDH } \\
\text { 6. Muscle glycogen }\end{array}$ & $\begin{array}{l}\text { 1. } P<0.05 \\
\text { 2. } P<0.01 \\
\text { 3. } P<0.05 \\
\text { 4. } P<0.05 \\
\text { 5. } P<0.05 \\
\text { 6. } P<0.05\end{array}$ \\
\hline
\end{tabular}




\begin{tabular}{|c|c|c|c|c|c|c|c|}
\hline Study (years) & $\begin{array}{l}\text { Species } \\
\text { Sex } \\
\text { Weight } \\
\text { N }\end{array}$ & Anesthetic & Model (method) & $\begin{array}{l}\text { Experimental group (drugs, } \\
\text { concentration, administration, } \\
\text { duration) }\end{array}$ & Control group & Outcome measure & $\begin{array}{l}\text { Intergroup } \\
\text { differences* }\end{array}$ \\
\hline Feng et al., 2009c & $\begin{array}{l}\text { SD rat } \\
M \\
180-220 \mathrm{~g} \\
40\end{array}$ & - & Horizontal treadmill exercise & $\begin{array}{l}\text { Ginsenoside Rb1 }(50 \mathrm{mg} / \mathrm{kg}) \\
\text { Ginsenoside Re(50 mg/kg) ig, } 14 \\
\text { days }\end{array}$ & $\begin{array}{l}\text { NS for same } \\
\text { volume }\end{array}$ & $\begin{array}{l}\text { 1. Hippocampus tissue } \\
\text { DA } \\
\text { 2. Hippocampus tissue } \\
\text { 5-HT } \\
\text { 3. Hippocampus tissue } \\
\text { GABA } \\
\text { 4. Hippocampus tissue } \\
\text { Ach }\end{array}$ & $\begin{array}{l}\text { 1. } 1 . P<0.05 \\
\text { 2. } P<0.05 \\
\text { 3. } P<0.01 \\
\text { 4. } P<0.01\end{array}$ \\
\hline Pan et al., 2010b & $\begin{array}{l}\text { SD rat } \\
\mathrm{M} \\
180-220 \mathrm{~g} \\
30\end{array}$ & - & Horizontal treadmill exercise & $\begin{array}{l}\text { Ginsenoside Rb1 ( } 50 \text { mg/kg) ig, } 14 \\
\text { days }\end{array}$ & $\begin{array}{l}\text { NS for same } \\
\text { volume }\end{array}$ & $\begin{array}{l}\text { 1. Serum MDA } \\
\text { 2. Serum SOD } \\
\text { 3. Liver MDA } \\
\text { 4. Liver SOD }\end{array}$ & $\begin{array}{l}\text { 1. } P<0.01 \\
\text { 2. } P<0.01 \\
\text { 3. } P<0.01 \\
\text { 4. 4. } P<0.01\end{array}$ \\
\hline Bao et al., 2016 & $\begin{array}{l}\text { ICR mice } \\
\mathrm{M} \\
18-22 \mathrm{~g} \\
240\end{array}$ & - & Forced Swimming Test & $\begin{array}{l}\text { GOP }(125,250,500 \text { mg/kg) ig, } 30 \\
\text { days }\end{array}$ & $\begin{array}{l}\text { Distilled water for } \\
\text { same volume }\end{array}$ & $\begin{array}{l}\text { 1. Mice body weight } \\
\text { 2. Swimming time in } \\
\text { mice } \\
\text { 3. Serum LDH } \\
\text { 4. Serum Urea } \\
\text { Nitrogen } \\
\text { 5. Hepatic Glycogen } \\
\text { 6. Blood Lactic Acid } \\
\text { 7. skeletal muscle } \\
\text { SOD } \\
\text { 8. skeletal muscles } \\
\text { CAT } \\
\text { 9. skeletal muscles } \\
\text { MDA } \\
\text { 10. NRF-1 } \\
\text { 11. TFAM } \\
\text { 12. mtDNA }\end{array}$ & $\begin{aligned} \text { 1. } P>0.05 \\
\text { 2. } P<0.01 \\
\text { 3. } P<0.01 \\
\text { 4. } P<0.01 \\
\text { 5. } P<0.01 \\
\text { 6. } P<0.01 \\
\text { 7. } P<0.01 \\
\text { 8. } P<0.01 \\
\text { 9. } P<0.01 \\
\text { 10. } P<0.05 \\
\text { 11. } P<0.05 \\
\text { 12. } P<0.05\end{aligned}$ \\
\hline Oh et al., 2015 & $\begin{array}{l}\text { ICR mice } \\
\mathrm{M} \\
26-28 \mathrm{~g} \\
49\end{array}$ & - & $\begin{array}{l}\text { weight-loaded swimming (WLS) test and } \\
\text { Rota-rod test }\end{array}$ & $\begin{array}{l}\text { PPD }(5,10 \mathrm{mg} / \mathrm{kg}) \\
\text { PPT }(5,10 \mathrm{mg} / \mathrm{kg}) \mathrm{ig}, 5 \text { days }\end{array}$ & $\begin{array}{l}\text { Distilled water for } \\
\text { same volume }\end{array}$ & $\begin{array}{l}\text { 1. Swimming time } \\
\text { 2. Serum } \\
\text { corticosterone } \\
\text { 3. Serum GLU } \\
\text { 4. Serum lactate } \\
\text { 5. Serum LDH } \\
\text { 6. Serum FFA } \\
\text { 7. Serum creatinine } \\
\text { 8. Serum } \\
\text { corticosterone } \\
\text { 9. Serum lactate } \\
\text { 10. Serum FFA } \\
\text { 11. Serum creatinine }\end{array}$ & $\begin{aligned} \text { 1. } P<0.05 \text { or } \\
P<0.01 \\
\text { 2. } P<0.05 \text { or } \\
P<0.01 \\
\text { 3. } P<0.05 \text { or } \\
P<0.01 \\
\text { 4. } P<0.05 \text { or } \\
P<0.01 \\
\text { 5. } P<0.05 \text { or } \\
P<0.01 \\
\text { 6. } P<0.05 \text { or } \\
P<0.01 \\
\text { 7. } P<0.05 \text { or } \\
P<0.01 \\
\text { 8. } P<0.01 \\
\text { 9. } P<0.05 \text { I }\end{aligned}$ \\
\hline
\end{tabular}




\section{Study (years)}

Species

Sex

Qi et al., $2014 \quad$ KM mice$$
20 \pm 2 \mathrm{~g}
$$$$
\begin{aligned}
& 20 \pm 29 \\
& 80
\end{aligned}
$$

Li et al., 2018

$$
\begin{aligned}
& \text { ICR mice } \\
& \mathrm{M}
\end{aligned}
$$$$
\text { 18-22 g }
$$$$
240
$$

$\begin{array}{ll}\text { Zhao et al., 2014 } & \text { KM mice } \\ & \text { F/M } \\ & 18-22 \mathrm{~g} \\ & 70 \\ & \\ \text { Gao et al., 2011 } & \text { mice } \\ & \text { M } \\ & \text { 18-22 g } \\ & \text { 120 } \\ & \\ \text { Ma et al., 2017 } & \text { ICR mice } \\ & \text { M } \\ \text { NG } \\ \text { 24 }\end{array}$

Forced swimming test

Forced swimming test
Model (method)

(a)

Experimental group (drugs,

Control group

Outcome measure concentration, administration, duration)

weight-loaded swimming (WLS) test

weight-loaded swimming (WLS) test

Forelimb Grip Strength/weight-loaded swimming (WLS) test

AGP $(125,250,500 \mathrm{mg} / \mathrm{kg})$ ig, 28
days

QOPs (225, 450, 900 mg/kg) ig, 30

\begin{tabular}{|c|c|c|}
\hline & & $\begin{array}{l}\text { 10. } \mathrm{P}<0.05 \\
\text { 11. } \mathrm{P}<0.05\end{array}$ \\
\hline \multirow{7}{*}{$\begin{array}{l}\text { Distilled water for } \\
\text { same volume }\end{array}$} & 1. Swimming time & 1. $P<0.01$ \\
\hline & 2. BLA & 2. $P<0.01$ \\
\hline & 3. SUN & 3. $P<0.01$ \\
\hline & 4. hepatic glycogen & 4. $P<0.01$ \\
\hline & 5. Serum GSH-Px & 5. $P<0.01$ \\
\hline & 6. Serum SOD & 6. $P<0.01$ \\
\hline & 7. Serum MDA & 7. $P<0.01$ \\
\hline \multirow{18}{*}{$\begin{array}{l}\text { NS for same } \\
\text { volume }\end{array}$} & 1. Swimming time & 1. $P<0.01$ \\
\hline & 2. Serum LDH & 2. $P<0.01$ \\
\hline & 3. BUN & 3. $P<0.01$ \\
\hline & 4. Hepatic glycogen & 4. $P<0.01$ \\
\hline & 5. BLA & 5. $P<0.01$ \\
\hline & 6. skeletal & 6. $P<0.01$ \\
\hline & & 7. $P<0.01$ \\
\hline & 7. skeletal & 8. $P<0.01$ \\
\hline & GSH-Px & 9. $P<0.01$ \\
\hline & 8. skeletal & 10. $P<0.01$ \\
\hline & MDA & 11. $P<0.01$ \\
\hline & 9. Na+-K+-ATPase & 12. $P<0.01$ \\
\hline & 10. Ca2+-Mg2+- & 13. $P<0.01$ \\
\hline & ATPase & 14. $P<0 . C$ \\
\hline & 11. SDH & \\
\hline & 12. NRF-1 & \\
\hline & 13. TFAM & \\
\hline & 14. mtDNA & \\
\hline \multirow{7}{*}{$\begin{array}{l}\text { Distilled water for } \\
\text { same volume }\end{array}$} & 1. Swimming time & 1. $P<0.01$ \\
\hline & 2. hypoxia tolerance & 2. $P<0.01$ \\
\hline & time & 3. $P<0.01$ \\
\hline & 3. BLA & 4. $P<0.01$ \\
\hline & 4. Hepatic glycogen & 5. $P<0.01$ \\
\hline & 5. muscle glycogen & 6. $P<0.01$ \\
\hline & 6. Serum BUN & \\
\hline \multirow{6}{*}{$\begin{array}{l}\text { NS for same } \\
\text { volume }\end{array}$} & 1. BLA & 1. $P<0.05$ \\
\hline & 2. Serum BUN & 2. $P<0.05$ \\
\hline & 3. Serum SOD & 3. $P>0.05$ \\
\hline & 4. Serum LDH & 4. $P>0.05$ \\
\hline & 5. Hepatic glycogen & 5. $P<0.01$ \\
\hline & 6. muscle glycogen & 6. $P<0.01$ \\
\hline \multirow{5}{*}{$\begin{array}{l}\text { Distilled water for } \\
\text { same volume }\end{array}$} & 1. Forelimb grip & 1. $P<0.01$ \\
\hline & strength & 2. $P<0.01$ \\
\hline & 2. Swimming time & 3. $P<0.01$ \\
\hline & 3. Serum Lactate & 4. $P<0.01$ \\
\hline & 4. Serum NH3 & 5. $P<0.0$ \\
\hline
\end{tabular}
days

Ginseng $(0.2 \mathrm{ml} / 10 \mathrm{~g})$

Red Ginseng $(0.2 \mathrm{ml} / 10 \mathrm{~g})$

mild property ginseng $(0.2 \mathrm{ml} / 10 \mathrm{~g})$ ig, 14 days

Ginseng $(1.25,2.5,5 \mathrm{mg} / \mathrm{kg})$ Red Ginseng $(1.25,2.5,5 \mathrm{mg} / \mathrm{kg})$

CMG extract $(5,25 \mathrm{mg} / \mathrm{kg}) \mathrm{ig}, 4$ weeks 


\begin{tabular}{|c|c|c|c|c|c|c|c|}
\hline Study (years) & $\begin{array}{c}\text { Species } \\
\text { Sex } \\
\text { Weight } \\
\text { N }\end{array}$ & Anesthetic & Model (method) & $\begin{array}{l}\text { Experimental group (drugs, } \\
\text { concentration, administration, } \\
\text { duration) }\end{array}$ & Control group & Outcome measure & $\begin{array}{l}\text { Intergroup } \\
\text { differences* }\end{array}$ \\
\hline & & & & & & $\begin{array}{l}\text { 5. Serum CK } \\
\text { 6. Serum GLU } \\
\text { 7. Serum BUN } \\
\text { 8. Hepatic glycogen } \\
\text { 9. muscle glycogen } \\
\text { 10. Serum LDH } \\
\text { 11. organ index }\end{array}$ & $\begin{array}{r}\text { 6. } P<0.01 \\
\text { 7. } P<0.01 \\
\text { 8. } P<0.01 \\
\text { 9. } P>0.05 \\
\text { 10. } P<0.01 \\
\text { 11. } P>0.05 \\
\text { 12. } P>0.05\end{array}$ \\
\hline Choi et al., 2011 & $\begin{array}{l}\text { ICR mice } \\
\mathrm{M} \\
17-20 \mathrm{~g} \\
40\end{array}$ & - & $\begin{array}{l}\text { restraint stress/Overcoming electroshock/ } \\
\text { Cold swimming test/Rota-rod test }\end{array}$ & $\begin{array}{l}\mathrm{RG}(50,100,200,400 \mathrm{mg} / \mathrm{kg}) \mathrm{ig}, 7 \\
\text { days }\end{array}$ & NR & $\begin{array}{l}\text { 1. Serum CORT } \\
\text { 2. Blood lactic acid }\end{array}$ & $\begin{array}{l}\text { 1. } P<0.05 \\
\text { 2. } P>0.05\end{array}$ \\
\hline Hwang et al., 2014 & $\begin{array}{l}\text { ICR mice } \\
\text { M } \\
\text { NG } \\
43\end{array}$ & - & aerobic running exercise & $R G(1 \mathrm{~g} / \mathrm{kg})$ ig, 2 weeks & $\begin{array}{l}\text { Distilled water for } \\
\text { same volume }\end{array}$ & $\begin{array}{l}\text { 1. 1.body weight } \\
\text { 2. Carbohydrate } \\
\text { Oxidation } \\
\text { 3. Fat Oxidation } \\
\text { 4. Serum Free fatty acid } \\
\text { 5. Serum Glucose } \\
\text { 6. SerumInsulin } \\
\text { 7. Liverglycogen } \\
\text { 8. Gastrocnemius-white } \\
\text { muscle glycogen } \\
\text { 9. Gastrocnemius-red } \\
\text { muscle glycogen }\end{array}$ & $\begin{array}{l}\text { 1. } P>0.05 \\
\text { 2. } P>0.05 \\
\text { 3. } P>0.05 \\
\text { 4. } P<0.05 \\
\text { 5. } P>0.05 \\
\text { 6. } P>0.05 \\
\text { 7. } P<0.05 \\
\text { 8. } P>0.05 \\
\text { 9. } P>0.05\end{array}$ \\
\hline Shi et al., 2016 & $\begin{array}{l}\text { KM mice } \\
\mathrm{M} \\
18-22 \mathrm{~g} \\
20\end{array}$ & - & $\begin{array}{l}\text { weight-loaded swimming (WLS) test and } \\
\text { Rota-rod test }\end{array}$ & $\begin{array}{l}\mathrm{BG}(1.65,3.30,4.95 \mathrm{~g} / \mathrm{kg}) \mathrm{ig}, 2 \\
\text { weeks }\end{array}$ & $\begin{array}{l}\text { Distilled water for } \\
\text { same volume }\end{array}$ & $\begin{array}{l}\text { 1. body weight } \\
\text { 2. pole-climbing time } \\
\text { 3. swimming time } \\
\text { 4. liverglycogen } \\
\text { 5. blood lactic acid } \\
\text { 6. Serum LDH }\end{array}$ & $\begin{array}{l}\text { 1. } P>0.05 \\
\text { 2. } P<0.001 \\
\text { 3. } P<0.001 \\
\text { 4. } P<0.01 \\
\text { 5. } P<0.01 \\
\text { 6. } P<0.01\end{array}$ \\
\hline
\end{tabular}

5-HT, 5-hydroxytryptamine; Ach, acetylcholine; BLA, Blood lactic acid; BUN, blood urea nitrogen; CK, creatine phosphokinase; CORT, corticosterone; DA, dopamine; GABA, gamma-aminobutyric acid; GLU, glucose; GSH-PX, glutathione peroxidase; LDH, lactic dehydrogenase; MDA, malondialdehyde; mIDNA, mitochondrial DNA; NRF-1, Nuclear respiratory factor 1; PTS, panaxtrol saponin; SDH, succinate dehydrogenase; SOD, superoxide dismutase; TG, triglyceride; TFAM, Mitochondrial transcription factor A; WGP, water-soluble ginseng polysaccharides; WGPA, ginseng pectin; WGPN, starch-like glucans. 
TABLE 4 | Statement of the characteristics of Panax ginseng C. A. Mey and its compounds(b).

Study Herb source $\begin{gathered}\text { Type of herbal Purity } \\ \text { or bioactive } \\ \text { compound }\end{gathered}$

\begin{tabular}{|c|c|c|c|c|}
\hline Xu et al., 2011 & $\begin{array}{l}\text { Panax ginseng C.A. } \\
\text { Mey }\end{array}$ & Ginseng proteins & $80 \%$ & not reported \\
\hline Wang et al., 2010 & $\begin{array}{l}\text { Panax ginseng C.A. } \\
\text { Mey }\end{array}$ & $\begin{array}{l}\text { Ginseng } \\
\text { polysaccharides }\end{array}$ & $\begin{array}{l}\text { not } \\
\text { reported }\end{array}$ & $\begin{array}{l}\text { the School of Life } \\
\text { Sciences, Northeast } \\
\text { Normal University, China }\end{array}$ \\
\hline Wang et al., 2013 & $\begin{array}{l}\text { Panax ginseng C.A. } \\
\text { Mey }\end{array}$ & $\begin{array}{l}\text { Ginseng pectin } \\
\text { polysaccharide }\end{array}$ & $\begin{array}{l}\text { not } \\
\text { reported }\end{array}$ & $\begin{array}{l}\text { the School of Life } \\
\text { Sciences, Northeast } \\
\text { Normal University, China }\end{array}$ \\
\hline Song et al., 2013 & $\begin{array}{l}\text { Panax ginseng C.A. } \\
\text { Mey }\end{array}$ & PTS & $\begin{array}{l}\text { not } \\
\text { reported }\end{array}$ & $\begin{array}{l}\text { Department of } \\
\text { Pathophysiology, Basic } \\
\text { Medical College of Jilin }\end{array}$ \\
\hline Xu et al., 2010 & $\begin{array}{l}\text { Panax ginseng C.A. } \\
\text { Mey }\end{array}$ & Ginsenoside Rb1 & $\begin{array}{l}\text { not } \\
\text { reported }\end{array}$ & $\begin{array}{l}\text { Shanghai Tongtian } \\
\text { Biotechnology Co., Ltd., } \\
\text { Shanghai, China }\end{array}$ \\
\hline
\end{tabular}

(lot number) Chemical

Structure or Composition

Not reported included-

$\begin{array}{ll}\text { reported } & \text { included- } \\ \text { (20081001) } & \text { HPLC }\end{array}$

reported included-

not reported

not
included

not reported

included-
HPLC

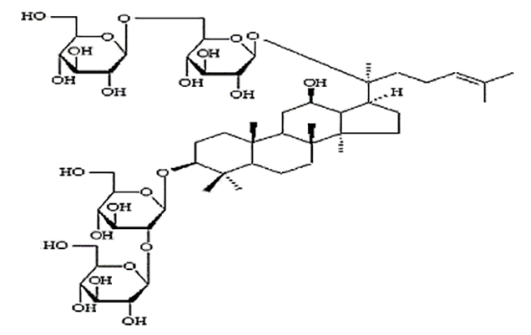

Feng et al., 2010a Panax ginseng C.A. Ginsenoside not Shanghai Tongtian

Mey Ginsenoside Rb1 reported Biotechnology Co., Lt

not reported Shanghai, China

included-
HPLC

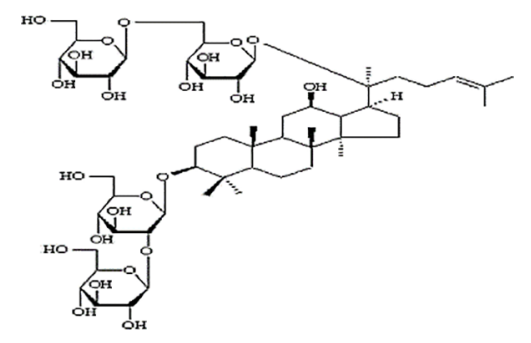
$\begin{array}{lllll}\text { Feng et al., 2010a } & \begin{array}{l}\text { Panax ginseng C.A. } \\ \text { Mey }\end{array} & \begin{array}{l}\text { Ginsenoside } \\ \text { Ginsenoside Re }\end{array} & \begin{array}{l}\text { not } \\ \text { reported }\end{array} & \begin{array}{l}\text { Shanghai Tongtian } \\ \text { Biotechnology Co., Ltd. }\end{array} \\ & & \end{array}$

not reported Shanghai, China

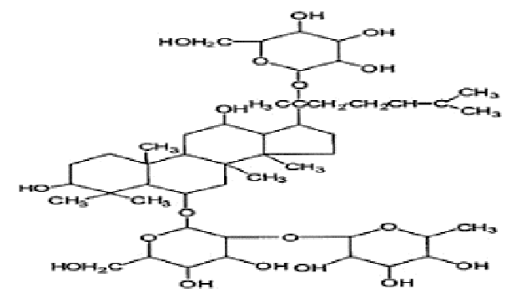


TABLE 4 | Continued

\begin{tabular}{|c|c|c|c|c|c|c|}
\hline Study & Herb source & \multirow{2}{*}{$\begin{array}{c}\begin{array}{c}\text { Type of herbal } \\
\text { or bioactive } \\
\text { compound }\end{array} \\
\text { Ginsenoside Rg1 }\end{array}$} & \multirow{2}{*}{$\begin{array}{l}\text { Purity } \\
98.59 \%\end{array}$} & \multirow{2}{*}{$\begin{array}{l}\text { Approach to achieving } \\
\begin{array}{l}\text { Dingguo Biotechnology } \\
\text { Co., Ltd., China }\end{array}\end{array}$} & \multirow{2}{*}{$\begin{array}{l}\begin{array}{c}\text { Quality control } \\
\text { (lot number) }\end{array} \\
\text { not reported }\end{array}$} & \multirow{2}{*}{$\begin{array}{l}\begin{array}{c}\text { Chemical } \\
\text { analysis }\end{array} \\
\text { included- } \\
\text { HPLC }\end{array}$} \\
\hline Liu, 2016 & $\begin{array}{l}\text { Panax ginseng C.A. } \\
\text { Mey }\end{array}$ & & & & & \\
\hline Feng et al., 2010b & $\begin{array}{l}\text { Panax ginseng C.A. } \\
\text { Mey }\end{array}$ & $\begin{array}{l}\text { Ginsenoside Rb1 } \\
\text { Ginsenoside Rg1 }\end{array}$ & $\begin{array}{l}\text { not } \\
\text { reported }\end{array}$ & $\begin{array}{l}\text { Shanghai Tongtian } \\
\text { Biotechnology Co., Ltd., } \\
\text { Shanghai, China }\end{array}$ & not reported & $\begin{array}{l}\text { included- } \\
\text { HPLC }\end{array}$ \\
\hline Wang et al., 2015 & $\begin{array}{l}\text { Panax ginseng C.A. } \\
\text { Mey }\end{array}$ & Ginsenoside Rg1 & $98 \%$ & $\begin{array}{l}\text { Department of Organic } \\
\text { Chemistry, Basic Medical } \\
\text { College of Jilin University, } \\
\text { Jilin, China }\end{array}$ & not reported & $\begin{array}{l}\text { included- } \\
\text { HPLC }\end{array}$ \\
\hline Yao, 2016 & $\begin{array}{l}\text { Panax ginseng C.A. } \\
\text { Mey }\end{array}$ & ginsenoside & $\begin{array}{l}\text { not } \\
\text { reported }\end{array}$ & not reported & not reported & $\begin{array}{l}\text { not } \\
\text { included }\end{array}$ \\
\hline Liu et al., 2015 & $\begin{array}{l}\text { Panax ginseng C.A. } \\
\text { Mey }\end{array}$ & Ginsenoside & $>80 \%$ & $\begin{array}{l}\text { Xi'an Ruiying } \\
\text { Biotechnology Co., Ltd., } \\
\text { Xi'an, China }\end{array}$ & $\begin{array}{l}\text { reported } \\
\text { (RYRS20140325) }\end{array}$ & $\begin{array}{l}\text { not } \\
\text { included }\end{array}$ \\
\hline Feng et al., 2009b & $\begin{array}{l}\text { Panax ginseng C.A. } \\
\text { Mey }\end{array}$ & Ginsenoside Re & $\begin{array}{l}\text { not } \\
\text { reported }\end{array}$ & $\begin{array}{l}\text { Shanghai Tongtian } \\
\text { Biotechnology Co., Ltd., } \\
\text { Shanghai, China }\end{array}$ & $\begin{array}{l}\text { Reported- } \\
\text { according to } \\
\text { (07120623) }\end{array}$ & $\begin{array}{l}\text { included- } \\
\text { HPLC }\end{array}$ \\
\hline
\end{tabular}


TABLE 4 | Continued

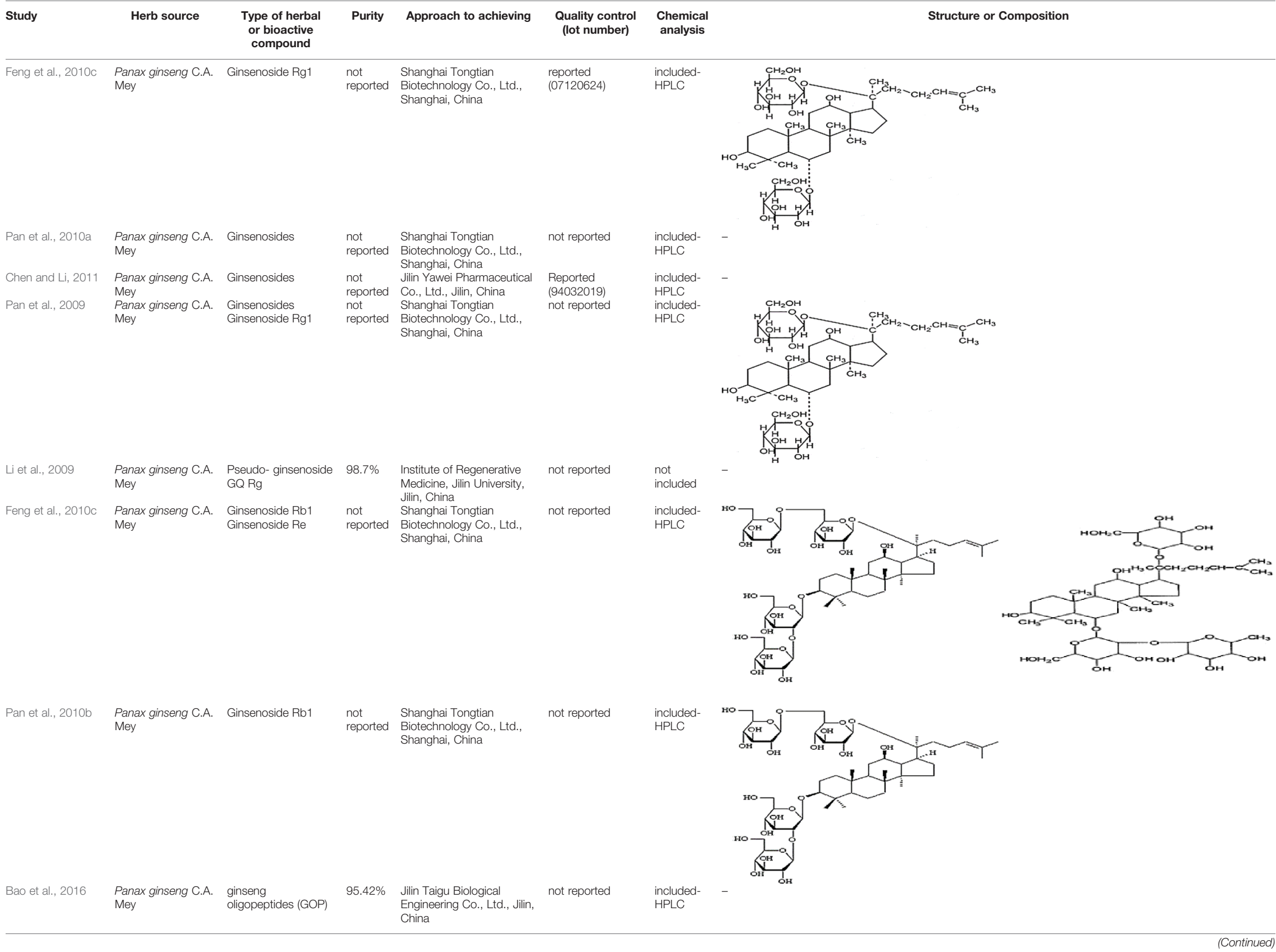


TABLE 4 | Continued

\begin{tabular}{|c|c|c|c|c|c|c|c|}
\hline Study & Herb source & $\begin{array}{l}\text { Type of herbal } \\
\text { or bioactive } \\
\text { compound }\end{array}$ & Purity & Approach to achieving & $\begin{array}{l}\text { Quality control } \\
\text { (lot number) }\end{array}$ & $\begin{array}{l}\text { Chemical } \\
\text { analysis }\end{array}$ & Structure or Composition \\
\hline Oh et al., 2015 & $\begin{array}{l}\text { Panax ginseng C.A. } \\
\text { Mey }\end{array}$ & $\begin{array}{l}\text { 20(S)- } \\
\text { Protopanaxadiol } \\
\text { (PPD) } \\
\text { 20(S)- } \\
\text { Protopanaxatriol } \\
\text { (PPT) }\end{array}$ & $\geq 98 \%$ & $\begin{array}{l}\text { the College of Pharmacy, } \\
\text { Kyung HeeUniversity, } \\
\text { Geumsan, Korea }\end{array}$ & $\begin{array}{l}\text { reported } \\
\text { (KHUP201409201) }\end{array}$ & $\begin{array}{l}\text { Included- } \\
\text { HPLC }\end{array}$ & \\
\hline Qi et al., 2014 & Panaxquinquefolium & $\begin{array}{l}\text { American ginseng } \\
\text { proteins (AGP) }\end{array}$ & $80 \%$ & Agilent Technologies, USA & not reported & $\begin{array}{l}\text { included- } \\
\text { HPLC }\end{array}$ & - \\
\hline Li et al., 2018 & Panaxquinquefolium & $\begin{array}{l}\text { small-molecule } \\
\text { oligopeptides } \\
\text { isolated from } \\
\text { American ginseng } \\
\text { (QOPs) }\end{array}$ & $96.46 \%$ & $\begin{array}{l}\text { SinoMed Peptide Valley } \\
\text { Bioengineering Co., Ltd., } \\
\text { China }\end{array}$ & $\begin{array}{l}\text { reported } \\
\text { (CN105154509A) }\end{array}$ & $\begin{array}{l}\text { included- } \\
\text { HPLC }\end{array}$ & - \\
\hline Zhao et al., 2014 & Red ginseng & $\begin{array}{l}\text { Ginseng } \\
\text { Red Ginseng } \\
\text { mild property } \\
\text { ginseng }\end{array}$ & $\begin{array}{l}\text { not } \\
\text { reported }\end{array}$ & not reported & not reported & $\begin{array}{l}\text { not } \\
\text { included }\end{array}$ & $\begin{array}{l}\text { Red GinsengContain } 4.015 \% \text { ginsenosides (Rg1 } 0.262 \% \text {, Re } 0.145 \% \text {, Rf } 0.151 \% \text {, Rh1 } 0.284 \% \text {, Rg2 } 0.402 \% \text {, } \\
\text { Rb1 0.394\%, Rc 0.318\%, Rb2 } 0.247 \% \text {, Rb3 0.465\%, Rd 0.437\%, Rg3 0.412\%, Rk1 0.194\%, Rg5 0.304\%) } \\
\text { HPLC data from Kim et al., } 2007\end{array}$ \\
\hline Gao et al., 2011 & $\begin{array}{l}\text { Panax ginseng C.A. } \\
\text { Mey }\end{array}$ & $\begin{array}{l}\text { Ginseng } \\
\text { Red Ginseng }\end{array}$ & $\begin{array}{l}\text { not } \\
\text { reported }\end{array}$ & not reported & not reported & $\begin{array}{l}\text { not } \\
\text { included }\end{array}$ & $\begin{array}{l}\text { Red GinsengContain 4.015\% ginsenosides (Rg1 0.262\%, Re } 0.145 \% \text {, Rf } 0.151 \% \text {, Rh1 } 0.284 \% \text {, Rg2 } 0.402 \% \text {, } \\
\text { Rb1 0.394\%, Rc 0.318\%, Rb2 0.247\%, Rb3 0.465\%, Rd 0.437\%, Rg3 0.412\%, Rk1 0.194\%, Rg5 0.304\%) } \\
\text { HPLC data from Kim et al., } 2007\end{array}$ \\
\hline Ma et al., 2017 & $\begin{array}{l}\text { Panax ginseng C.A. } \\
\text { Mey }\end{array}$ & $\begin{array}{l}\text { Changbai Mountain } \\
\text { Ginseng(CMG) }\end{array}$ & $\begin{array}{l}\text { not } \\
\text { reported }\end{array}$ & not reported & not reported & $\begin{array}{l}\text { included- } \\
\text { HPLC }\end{array}$ & $\begin{array}{l}\text { Contain 11.5\% ginsenosides (Ra1 0.46\%, Ra2 0.69\%, Rb1 0.22\%, Rb2 0.94\%, Rb3 1.22\%, Rc 0.33\%, Rd } \\
\text { 1.54\%, Re 0.11\%, Rf 1.14\%, Rg1 0.2\%, Ro 1.19\%, 20-glc-G-Rf 1.60\%, R1 0.71\%, R2 1.15\%) HPLC data } \\
\text { from Wang et al. } 2017\end{array}$ \\
\hline Choi et al., 2011 & Red ginseng & Ren Ginseng & $\begin{array}{l}\text { not } \\
\text { reported }\end{array}$ & $\begin{array}{l}\text { Korea Ginseng Corp., } \\
\text { Seoul, Korea }\end{array}$ & not reported & $\begin{array}{l}\text { included- } \\
\text { HPLC }\end{array}$ & $\begin{array}{l}\text { Contain } 4.015 \% \text { ginsenosides (Rg1 0.262\%, Re 0.145\%, Rf 0.151\%, Rh1 0.284\%, Rg2 } 0.402 \% \text {, Rb1 } \\
\text { 0.394\%, Rc 0.318\%, Rb2 0.247\%, Rb3 0.465\%, Rd 0.437\%, Rg3 0.412\%, Rk1 0.194\%, Rg5 0.304\%) } \\
\text { HPLC data from Kim et al., } 2007\end{array}$ \\
\hline Hwang et al., 2014 & Red ginseng & Ren Ginseng & $\begin{array}{l}\text { not } \\
\text { reported }\end{array}$ & $\begin{array}{l}\text { Korea Ginseng Corp., } \\
\text { Seoul, Korea }\end{array}$ & not reported & $\begin{array}{l}\text { included- } \\
\text { HPLC }\end{array}$ & $\begin{array}{l}\text { Contain 1.964\% ginsenosides(Rg1 0.071\%, Re 0.093\%, Rf 0.121\%, Rf1 0.078\%, Rg2(s) 0.192\%, Rg2 } \\
\text { O.129\%, Rb1 0.462\%, Rc 0.241\%, Rb2 0.183\%, Rd 0.089\%, Rg3(s) 0.214\%, Rg3 0.091\%) HPLC data from } \\
\text { Hwang et al., } 2014\end{array}$ \\
\hline Shi et al., 2016 & Black ginseng & black Ginseng & $\begin{array}{l}\text { not } \\
\text { reported }\end{array}$ & $\begin{array}{l}\text { HunchunXuzhu Trading } \\
\text { Co., Ltd., Jilin, China }\end{array}$ & not reported & $\begin{array}{l}\text { not } \\
\text { included }\end{array}$ & $\begin{array}{l}\text { Contain 3.341\% ginsenosides (Rg1 0.232\%, Re } 0.186 \% \text {, Rf } 0.158 \% \text {, Rb1 } 0.184 \% \text {, Rc } 0.145 \% \text {, Rb2 } 0.174 \% \text {, } \\
\text { Rd 0.169\%, Rg6 0.138\%, F4 0.127\%, Rk3 } 0.162 \% \text {, Rh4 0.102\%, 20(S)-Rg3 0.173\%, 20(R)-Rg3 0.121\%, } 20 \\
\text { (S)-Rs3 0.146\%, 20(R)-Rs3 0.213\%, Rk1 0.178\%, Rg5 0.321\%, Rs5 0.225\%, Rs4 0.187\%) HPLC data from } \\
\text { Sun et al., 2009 }\end{array}$ \\
\hline
\end{tabular}


TABLE 5 | The methodological quality of included randomized control trials.

\begin{tabular}{lllllllll}
\hline included studies & A & B & C & D & E & F & G & Total score \\
\hline Engels et al., 1996 & + & $?$ & + & $?$ & + & $?$ & + & \\
Engels et al., 2001 & + & $?$ & + & $?$ & + & $?$ & + & $4+$ \\
Engels et al., 2003 & + & $?$ & + & $?$ & + & $?$ & + & $4+$ \\
Hartz et al., 2004 & + & + & + & + & + & $?$ & + & $4+$ \\
Hyeong-Geug et al., 2013 & + & + & + & $?$ & + & + & + & $6+$ \\
Kim et al., 2016 & + & $?$ & + & $?$ & + & + & + & $5+$ \\
Lee et al., 2016 & + & + & + & + & + & + & + & $7+$ \\
Gal et al., 1996 & + & $?$ & + & $?$ & + & $?$ & + & $4+$
\end{tabular}

$A$, random sequence generation; $B$, allocation concealment; $C$, blinding of participants and personnel; $D$, blinding of outcome assessment; $E$, incomplete outcome data; $F$, selective reporting; $G$, other sources of bias.

et al., 2009; Feng et al., 2010a; Feng et al., 2010b; Feng et al., 2010c; Pan et al., 2010a; Pan et al., 2010b; Xu et al., 2010; Chen and Li, 2011; Gao et al., 2011; Choi et al., 2011; Song et al., 2013; Hwang et al., 2014; Qi et al., 2014; Wang et al., 2014; Zhao et al., 2014; Oh et al., 2015; Bao et al., 2016; Liu, 2016; Shi et al., 2016; Ma et al., 2017; Zheng et al., 2017; Delgado et al., 2019) described control of temperature. Twenty-four studies (Feng et al., 2009a; Feng et al., 2009b; Li et al., 2009; Pan et al., 2009; Feng et al., 2010a; Feng et al., 2010b; Feng et al., 2010c; Pan et al., 2010a; Pan et al., 2010b; Choi et al., 2011; Gao et al., 2011; Xu et al., 2011; Song et al., 2013; Hwang et al., 2014; Qi et al., 2014; Wang et al., 2014; Zhao et al., 2014; Liu et al., 2015; Oh et al., 2015; Bao et al., 2016; Liu, 2016; Ma et al., 2017; Zheng et al., 2017; Delgado et al., 2019) declared no potential conflict of interests, and 9 studies (Choi et al., 2011; Hwang et al., 2014; Qi et al., 2014; Wang et al., 2014; Oh et al., 2015; Bao et al., 2016; Ma et al., 2017; Zheng et al., 2017; Delgado et al., 2019) reported compliance with animal welfare regulations. The methodological quality is concluded in Table 6.

\section{Effectiveness}

\section{Randomized Control Trials}

Five studies (Gal et al., 1996; Hartz et al., 2004; Hyeong-Geug et al., 2013; Kim et al., 2016; Lee et al., 2016) described the treatment of CFS patients. These studies utilized different scales of fatigue to measure therapeutic effect. Therefore, the meta-analysis was not conducted because of different evaluation criteria. All studies showed significant difference in their respective fatigue scales for PGC relative to placebo. Three studies (Gal et al., 1996; Hartz et al., 2004; Kim et al., 2016) reported clinical effect, which according to different self-reported fatigue scales. Meta-analysis of 3 studies (Gal et al., 1996; Hartz et al., 2004; Kim et al., 2016) found significant difference in clinical effect for PGC relative to placebo ( $\mathrm{nT} / \mathrm{nC}=181 / 133, \mathrm{OR}=1.31,95 \% \mathrm{CI}: 1.17$ to $1.46, \mathrm{P}<0$ 00001; heterogeneity $\left.\chi^{2}=1.94, \mathrm{df}=2(\mathrm{P}=0.38), \mathrm{I}^{2}=0 \%\right)$ (Figure 2A). Meta-analysis of 5 studies (Gal et al., 1996; Hartz et al., 2004; Hyeong-Geug et al., 2013; Kim et al., 2016; Lee et al., 2016) showed there was no significant difference in the frequency of adverse events between the groups ( $\mathrm{nT} / \mathrm{nC}=293 / 297, \mathrm{OR}=1.09$, 95\% CI: 0.48 to $2.46, \mathrm{P}=0.83$, heterogeneity $\chi^{2}=6.99, \mathrm{df}=4$ $\left.(\mathrm{P}=0.14), \mathrm{I}^{2}=0 \%\right)$ (Figure 2B).

Three studies (Engels et al., 1996; Engels et al., 2001; Engels et al., 2003) described the effect of PGC on physical performance
TABLE 6 | Risk of bias of the included animal studies.

\begin{tabular}{|c|c|c|c|c|c|c|c|c|c|c|c|}
\hline Study & A & B & C & D & $\mathbf{E}$ & $\mathbf{F}$ & G & $\mathbf{H}$ & I & J & Total \\
\hline Xu et al., 2011 & + & $?$ & + & $?$ & $?$ & + & + & $?$ & $?$ & + & $5+$ \\
\hline Wang et al., 2010 & + & + & $?$ & $?$ & $?$ & + & + & $?$ & + & + & $6+$ \\
\hline Wang et al., 2013 & + & + & $?$ & $?$ & $?$ & + & + & $?$ & + & + & $6+$ \\
\hline Song et al., 2013 & + & + & + & $?$ & $?$ & + & + & $?$ & $?$ & + & $6+$ \\
\hline Xu et al., 2010 & + & + & + & $?$ & $?$ & + & + & $?$ & $?$ & $?$ & $5+$ \\
\hline Feng et al., $2010 a$ & + & + & + & $?$ & $?$ & + & + & $?$ & $?$ & + & $6+$ \\
\hline Feng et al., 2009a & + & + & + & $?$ & $?$ & + & + & $?$ & $?$ & + & $6+$ \\
\hline Liu, 2016 & - & + & + & $?$ & $?$ & + & + & $?$ & $?$ & + & $4+$ \\
\hline Feng et al., 2010b & + & + & + & $?$ & $?$ & + & + & $?$ & $?$ & + & $6+$ \\
\hline Wang et al., 2015 & + & $?$ & + & $?$ & $?$ & + & + & $?$ & $?$ & $?$ & $4+$ \\
\hline Yao, 2016 & + & $?$ & + & $?$ & $?$ & + & + & $?$ & $?$ & $?$ & $4+$ \\
\hline Liu et al., 2015 & + & $?$ & + & $?$ & $?$ & + & + & $?$ & $?$ & + & $5+$ \\
\hline Feng et al., 2010b & + & ? & + & $?$ & $?$ & + & + & $?$ & $?$ & $?$ & $4+$ \\
\hline Feng et al., 2010c & + & + & + & $?$ & $?$ & + & + & $?$ & $?$ & + & $6+$ \\
\hline Pan et al., 2010a & + & + & + & $?$ & $?$ & + & + & $?$ & $?$ & + & $6+$ \\
\hline Pan et al., 2010a & + & + & + & $?$ & $?$ & + & + & $?$ & $?$ & $?$ & $5+$ \\
\hline Pan et al., 2009 & + & + & + & $?$ & $?$ & + & + & $?$ & $?$ & + & $6+$ \\
\hline Li et al., 2009 & + & $?$ & + & $?$ & $?$ & + & + & $?$ & $?$ & + & $5+$ \\
\hline Feng et al., 2009c & + & + & + & $?$ & $?$ & + & + & $?$ & $?$ & + & $6+$ \\
\hline Pan et al., 2010b & + & + & + & $?$ & $?$ & + & + & $?$ & $?$ & + & $6+$ \\
\hline Bao et al., 2016 & + & + & + & $?$ & $?$ & + & + & $?$ & + & + & $7+$ \\
\hline Oh et al., 2015 & + & + & + & $?$ & $?$ & + & + & $?$ & + & + & $7+$ \\
\hline Qi et al., 2014 & + & + & + & $?$ & $?$ & + & + & $?$ & + & + & $7+$ \\
\hline Li et al., 2018 & + & + & + & $?$ & $?$ & + & + & $?$ & + & + & $7+$ \\
\hline Zhao et al., 2014 & + & + & + & $?$ & $?$ & + & + & $?$ & $?$ & + & $6+$ \\
\hline Gao et al., 2011 & + & + & + & $?$ & $?$ & + & + & $?$ & $?$ & + & $6+$ \\
\hline Ma et al., 2017 & + & + & + & $?$ & $?$ & + & + & $?$ & + & + & $7+$ \\
\hline Choi et al., 2011 & + & + & $?$ & $?$ & $?$ & + & + & $?$ & + & + & $6+$ \\
\hline Hwang et al., 2014 & + & + & + & $?$ & $?$ & + & + & $?$ & + & + & $7+$ \\
\hline Shi et al., 2016 & + & + & + & $?$ & $?$ & + & + & $?$ & $?$ & $?$ & $5+$ \\
\hline
\end{tabular}

Studies fulfilling the criteria of the following: (A) peer reviewed publication; (B) control of temperature; (C) random allocation to treatment or control; $(D)$ blinded induction of model; (E) blinded assessment of outcome; (F) use of anesthetic without significant intrinsic neuroprotective activity; (G) animal model (aged or female involved); (H) sample size calculation; (I) compliance with animal welfare regulations; (J) statement of potential conflict of interests.

recovery of healthy adults after exercise fatigue. Meta-analysis of 3 studies (Engels et al., 1996; Engels et al., 2001; Engels et al., 2003) found significant difference in heart rate recovery after exercise compared the PGC with placebo $(\mathrm{nT} / \mathrm{nC}=35 / 30 ; \mathrm{FE}=$ -5.73 ; $95 \% \mathrm{CI},-9.22$ to $-2.55 ; P=0.001$; heterogeneity $\chi^{2}=3.80$, $\left.\mathrm{df}=2(P=0.15), I^{2}=47 \%\right)$ (Figure 2C).

\section{Animal Studies}

\section{The Swimming Time}

Meta-analysis of 8 studies (Li et al., 2009; Xu et al., 2011; Zhao et al., 2014; Liu et al., 2015; Wang et al., 2015; Shi et al., 2016; Yao, 2016; Ma et al., 2017) showed PGC significantly increased the swimming time in WLST compared with control $(\mathrm{n}=98$, SMD $=2.21,95 \%$ CI: 1.83 to $2.59, \mathrm{P}<0$ 00001; heterogeneity: $\chi^{2}=$ 18.96, $\left.\mathrm{df}=7(\mathrm{P}=0.008), \mathrm{I}^{2}=63 \%\right)$. Owing to obvious heterogeneity, we used sensitivity analyses and removed one study (Li et al., 2009) that trained the mice before the swimming test. Meta-analysis of 7 studies showed a significant effect of PGC in increasing the swimming time in WLST compared with control $(\mathrm{n}=78, \mathrm{SMD}=2.60,95 \% \mathrm{CI}: 2.14$ to $3.06, \mathrm{P}<0$ 00001; heterogeneity: $\chi^{2}=9.75$, df $\left.=6(\mathrm{P}=0.14), \mathrm{I}^{2}=38 \%\right)$ (Figure 3A). Five studies (Choi et al., 2011; Song et al., 2013; Qi 
A

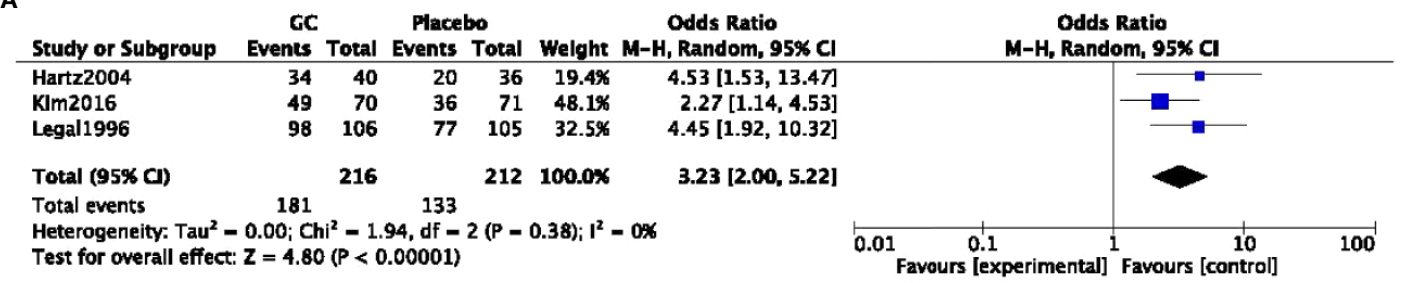

B

$\begin{array}{cccc} & \text { GC } & \text { Placebo } & \text { Odds Ratio } \\ \text { Study or Subgroup } & \text { Events } & \text { Total } & \text { Events Total } \\ \text { Welght } & \text { M-H, Random, 95\% CI }\end{array}$

$\begin{array}{cccc} & \text { GC } & \text { Placebo } & \text { Odds Ratio } \\ \text { Study or Subgroup } & \text { Events } & \text { Total } & \text { Events Total } \\ \text { Welght } & \text { M-H, Random, 95\% Cl }\end{array}$ $\begin{array}{lllllll}\text { Hartz2004 } & 8 & 47 & 4 & 49 & 23.1 \% & 2.31[0.65,8.25]\end{array}$ $\begin{array}{lllllll}\text { Kim2013 } & 1 & 29 & 0 & 30 & 5.7 \% & 3.21[0.13,82.07]\end{array}$ $\begin{array}{lllllll}K I m 2016 & 9 & 81 & 18 & 83 & 32.9 \% & 0.45[0.19,1.07]\end{array}$ Lee2016 Legal1996

Total (95\% Cl)

$14 \quad 110$

$1 \quad 26 \quad 5.7 \%$ $0.32[0.01,8.24]$
$1.62[0.67,3.92]$

$293 \quad 297100.0 \% \quad 1.09[0.48,2.49]$ Total events
Heterogenelty: $\mathrm{Tau}^{2}=0.34 ; \mathrm{ChI}^{2}=6.99, \mathrm{df}=4(\mathrm{P}=0.14) ; \mathrm{I}^{2}=43 \%$ Test for overall effect: $Z=0.21(P=0.83)$

$1.09[0.48,2.49]$

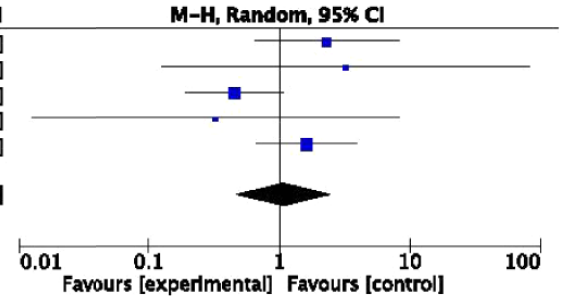

Odds Ratio

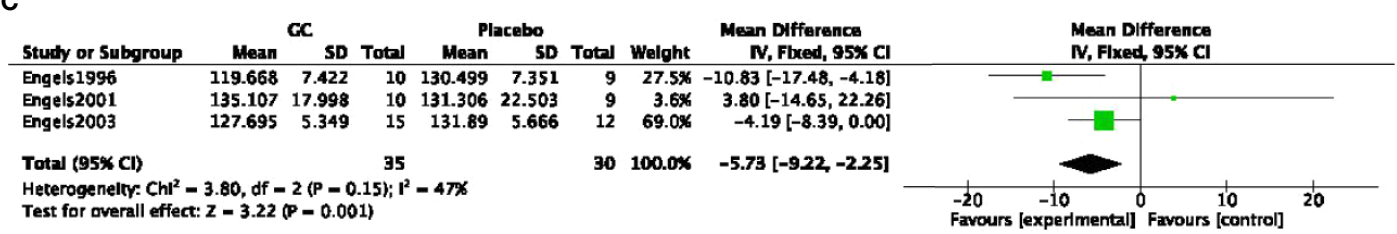

FIGURE 2 | (A) Clinical effect of Panax ginseng C. A. Mey and its compounds versus Placebo. (B) Adverse events of Panax ginseng C. A. Mey and its compounds versus Placebo. (C) Heart rate recovery of Panax ginseng C. A. Mey and its compounds versus Placebo.

A

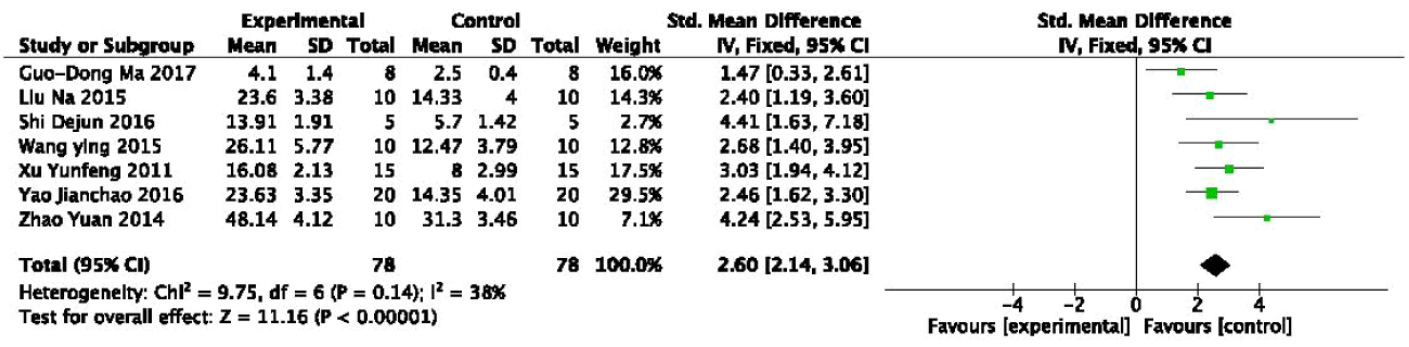

B

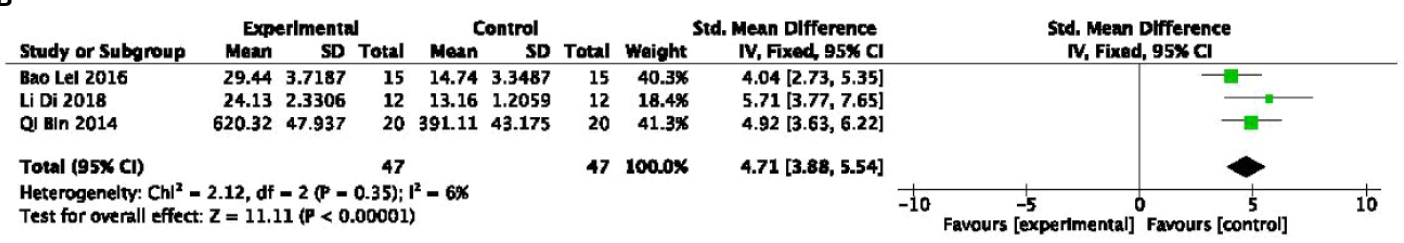

FIGURE 3 | (A) Forced swimming test of Panax ginseng C. A. Mey and its compounds versus Control. (B) Weight-loaded swimming test of Panax ginseng C. A. Mey and its compounds versus Control. 
et al., 2014; Bao et al., 2016; Delgado et al., 2019) found that PGC was more effective in increasing the swimming time in FST than that of control ( $\mathrm{n}=67, \mathrm{SMD}=2.78,95 \% \mathrm{CI}$ : 2.24 to $3.32, \mathrm{P}<0$ 00001; heterogeneity: $\left.\chi^{2}=41.15, \mathrm{df}=4(\mathrm{P}<000001), \mathrm{I}^{2}=90 \%\right)$. Two studies (Choi et al., 2011; Song et al., 2013) may be considered as the probable source of the heterogeneity, in which 1 study (Song et al., 2013) utilized rats as experimental subjects and one study (Choi et al., 2011) utilized crude products of PG. After getting rid of these 2 studies, there was showing that PGC increasing the swimming time in FST $(n=47, \mathrm{SMD}=4.71$, 95\% CI: 3.88 to 5.54, $\mathrm{P}<0$ 00001; heterogeneity: $\chi^{2}=2.12, \mathrm{df}=2$ $\left.(\mathrm{P}=0.35), \mathrm{I}^{2}=6 \%\right)$ (Figure 3B).

\section{Serum Biochemical Parameters}

Meta-analysis of 3 studies (Qi et al., 2014; Wang et al., 2014; Zheng et al., 2017) showed PGC was significant for increasing level of GSH-Px compared with control ( $\mathrm{n}=47, \mathrm{SMD}=2.16,95 \%$ CI: 1.49 to $3.02, \mathrm{P}<0$ 00001; heterogeneity: $\chi^{2}=46.87, \mathrm{df}=2$ $\left.(\mathrm{P}<000001), \mathrm{I}^{2}=96 \%\right)$. After sensitivity analyses, we removed 1 study (Qi et al., 2014) that utilized ginseng protein as intervention. Meta-analysis of the rest of 2 studies found that PGC significantly increased the level of GSH-Px compared with control ( $\mathrm{n}=16, \mathrm{SMD}=1.42,95 \% \mathrm{CI}$ : 0.61 to $2.22, \mathrm{P}=0.0005$; heterogeneity: $\left.\chi^{2}=0.37, \mathrm{df}=1(\mathrm{P}=0.54), \mathrm{I}^{2}=0 \%\right)$ (Figure 4A). Compared with controls, meta-analysis of 5 studies (Song et al., 2013; Wang et al., 2014; Wang et al., 2015; Oh et al., 2015; Ma et al., 2017) showed that PGC significantly increased level of GLU ( $\mathrm{n}=41, \mathrm{SMD}=1.69,95 \% \mathrm{CI}: 1.16$ to $2.23, \mathrm{P}<000001$; heterogeneity: $\left.\chi^{2}=4.65, \mathrm{df}=4(\mathrm{P}=0.32), \mathrm{I}^{2}=14 \%\right)$ (Figure 4B); 11 studies (Li et al., 2009; Gao et al., 2011; Xu et al., 2011; Song et al., 2013; Qi et al., 2014; Zhao et al., 2014; Liu et al., 2015; Wang et al., 2015; Bao et al., 2016; Yao, 2016; Ma et al., 2017; Delgado et al., 2019) for reducing level of BUN ( $\mathrm{n}=160, \mathrm{SMD}=$ -1.05 ; $95 \% \mathrm{CI},-1.29$ to $-0.80 ; P<000001$; heterogeneity: $\chi^{2}=$ 21.75, $\mathrm{df}=10\left(P<0\right.$ 00001), $\left.\mathrm{I}^{2}=49 \%\right)$ (Figure 4C); 15 studies $(\mathrm{Li}$ et al., 2009; Choi et al., 2011; Gao et al., 2011; Xu et al., 2011; Song et al., 2013; Qi et al., 2014; Zhao et al., 2014; Liu et al., 2015; Oh et al., 2015; Wang et al., 2015; Bao et al., 2016; Liu, 2016; Shi et al., 2016; Yao, 2016; Delgado et al., 2019) for reducing level of BLA $(\mathrm{n}=186 ; \mathrm{SMD}=-1.25 ; 95 \% \mathrm{CI},-1.48$ to $-1.02 ; \mathrm{P}<000001$; heterogeneity: $\chi^{2}=24.26$; $\left.\mathrm{df}=14(\mathrm{P}=0.04) ; \mathrm{I}^{2}=42 \%\right)$ (Figure 4D); four studies (Liu et al., 2015; Yao, 2016; Ma et al., 2017; Zheng et al., 2017) for reducing level of CK ( $\mathrm{n}=44$; SMD = -2.48 ; $95 \% \mathrm{CI},-3.07$ to -1.89 ; $\mathrm{P}<000001$; heterogeneity: $\chi^{2}=$ 4.22, $\left.\mathrm{df}=3(\mathrm{P}=0.24), \mathrm{I}^{2}=29 \%\right)$ (Figure 5A); five studies (Feng et al., 2009c; Pan et al., 2010a; Qi et al., 2014; Wang et al., 2014; Zheng et al., 2017) for reducing level of MDA ( $\mathrm{n}=44$; SMD = -2.86 ; $95 \% \mathrm{CI},-3.42$ to $-2.30 ; P<0$ 00001; heterogeneity, $\chi^{2}=$ 6.42 , df $\left.=3(P=0.17), I^{2}=38 \%\right)$ (Figure 5B); five studies (Feng et al., 2009c; Pan et al., 2010a; Qi et al., 2014; Wang et al., 2014; Zheng et al., 2017) for increasing level of SOD $(\mathrm{n}=56$; SMD = 2.35; 95\% CI, 1.84 to $2.86, P<000001$; heterogeneity: $\chi^{2}=4.86$, $\left.\mathrm{df}=4(\mathrm{P}=0.30), \mathrm{I}^{2}=18 \%\right)$ (Figure $5 \mathrm{C}$ ); four studies (Wang et al., 2014; Oh et al., 2015; Ma et al., 2017; Zheng et al., 2017) for reducing level of $\mathrm{LDH}(\mathrm{n}=29$; $\mathrm{SMD}=-2.28 ; 95 \% \mathrm{CI},-3.00$ to $-1.55, P<0$ 00001; heterogeneity: $\chi^{2}=3.76, \mathrm{df}=3(P=0.29), \mathrm{I}^{2}=$ 20\%) (Figure 5D). Six studies (Li et al., 2009; Liu et al., 2015; Bao et al., 2016; Shi et al., 2016; Yao, 2016; Delgado et al., 2019) found that PGC was more effective for increasing activity of LDH than that of control $(\mathrm{P}<0.05)$. No meta-analysis was carried out because of high heterogeneity.

\section{Skeletal Muscle Biochemical Parameters:}

Meta- analysis of 6 studies (Li et al., 2009; Feng et al., 2010b; Gao et al., 2011; Zhao et al., 2014; Wang et al., 2015; Ma et al., 2017) showed a significant effect of PGC for increasing level of muscle glycogen compared with control $(\mathrm{n}=68, \mathrm{SMD}=0.80,95 \% \mathrm{CI}$ : 0.43 to $1.17, \mathrm{P}<000001$; heterogeneity: $\chi^{2}=17.10$, df $=5$ $\left.(\mathrm{P}=0.004), \mathrm{I}^{2}=71 \%\right)$. After removing 1 studies (Feng et al., 2010b) that utilized rats as experimental subjects, meta- analysis of 5 studies (Li et al., 2009; Gao et al., 2011; Zhao et al., 2014; Wang et al., 2015; Ma et al., 2017) showed similar results $(n=58$, SMD $=0.63,95 \%$ CI: 0.25 to $1.01, P=0.001$; heterogeneity: $\left.\chi^{2}=6.14, \mathrm{df}=4(\mathrm{P}=0.19), \mathrm{I}^{2}=35 \%\right)$ (Figure 6A). Compared with control, meta-analysis of 9 studies (Feng et al., 2009c; Feng et al., 2010a; Feng et al., 2010b; Pan et al., 2010a; Pan et al., 2010b; Xu et al., 2010; Bao et al., 2016; Liu, 2016; Delgado et al., 2019) showed that PGC significantly improved activity of SOD $(n=97$, SMD $=2.61,95 \%$ CI: 2.19 to $3.03, \mathrm{P}<0$ 00001; heterogeneity: $\chi^{2}=$ $\left.23.71, \mathrm{df}=8(\mathrm{P}=0.003), \mathrm{I}^{2}=66 \%\right)$. Sensitivity analyses were conducted to explore potential sources of heterogeneity. After removing 1 trial (Pan et al., 2010b) that utilized crude products of PG, meta-analysis of 8 studies (Feng et al., 2009c; Feng et al., 2010a; Feng et al., 2010b; Pan et al., 2010a; Xu et al., 2010; Bao et al., 2016; Liu, 2016; Delgado et al., 2019) showed that PGC was still superior to the control ( $\mathrm{n}=87, \mathrm{SMD}=2.96,95 \% \mathrm{CI}: 2.49$ to $3.42, \mathrm{P}<000001$; heterogeneity: $\chi^{2}=12.32$, $\mathrm{df}=7$ ( $\left.\mathrm{P}=0.09\right)$, $\mathrm{I}^{2}=43 \%$ ) (Figure 6B). Compared with control group, metaanalysis of 2 studies (Liu, 2016; Bao et al., 2016) showed that PGC had significant effect to increase activity of CAT $(\mathrm{n}=25$, SMD $=1.90,95 \%$ CI: 1.21 to $2.59, \mathrm{P}<0$ 00001; heterogeneity: $\chi^{2}=$ $\left.1.05, \mathrm{df}=41(\mathrm{P}=0.31), \mathrm{I}^{2}=5 \%\right)$ (Figure 6C); 2 studies (Liu, 2016; Delgado et al., 2019) for improving activity of GSH-Px $(\mathrm{n}=22$, SMD $=2.01,95 \%$ CI: 1.26 to $2.77, \mathrm{P}<0$ 00001; heterogeneity: $\chi^{2}=$ $\left.0.47, \mathrm{df}=1(\mathrm{P}=0.49), \mathrm{I}^{2}=0 \%\right)$ (Figure 6D). The level of MDA was reported in 9 studies (Feng et al., 2009c; Feng et al., 2010b; Feng et al., 2010c; Pan et al., 2010a; Pan et al., 2010b; Xu et al., 2010; Bao et al., 2016; Liu, 2016; Delgado et al., 2019). There was significant decreasing in the level of MDA compared PGC with control $(\mathrm{P}<0.05)$. No meta-analysis was carried out because of high heterogeneity.

\section{Liver Biochemical Parameters}

Meta-analysis of 11 studies (Gao et al., 2011; Xu et al., 2011; Zhao et al., 2014; Qi et al., 2014; Liu et al., 2015; Wang et al., 2015; Shi et al., 2016; Yao, 2016; Bao et al., 2016; Ma et al., 2017; Delgado et al., 2019) showed that PGC had significant effects for increasing level of hepatic glycogen compared with control ( $\mathrm{n}=135$, SMD $=2.19,95 \%$ CI: 1.86 to $2.52, \mathrm{P}<000001$; heterogeneity: $\chi^{2}=58.77, \mathrm{df}=10\left(\mathrm{P}<0\right.$ 00001), $\mathrm{I}^{2}=$ $83 \%)$.Owing to obvious heterogeneity, we conducted subgroup analysis. These studies were divided into 4 subgroups according to different experiment interventions. Meta-analysis showed that there was significant difference in the level of glycogen between 
A

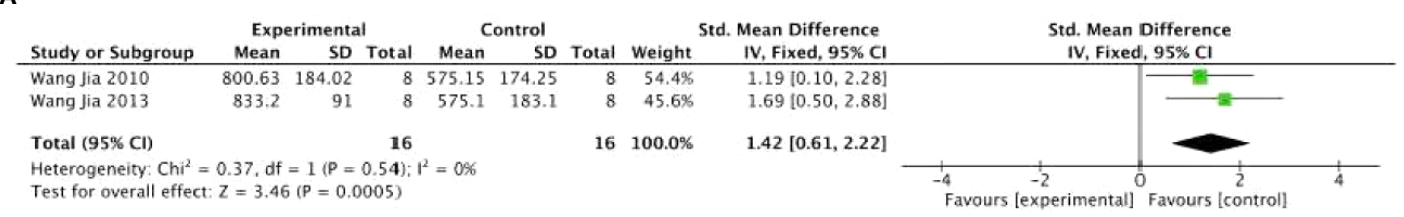

B

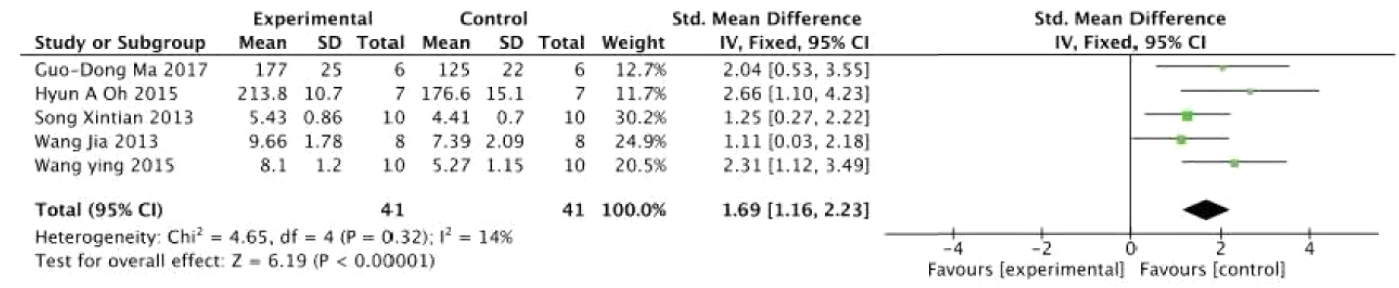

C

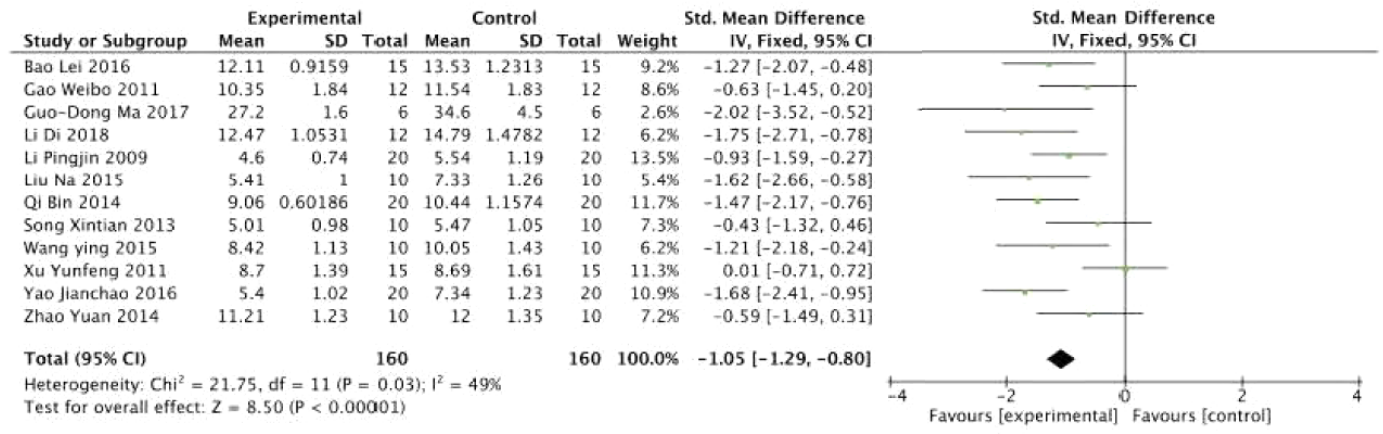

D

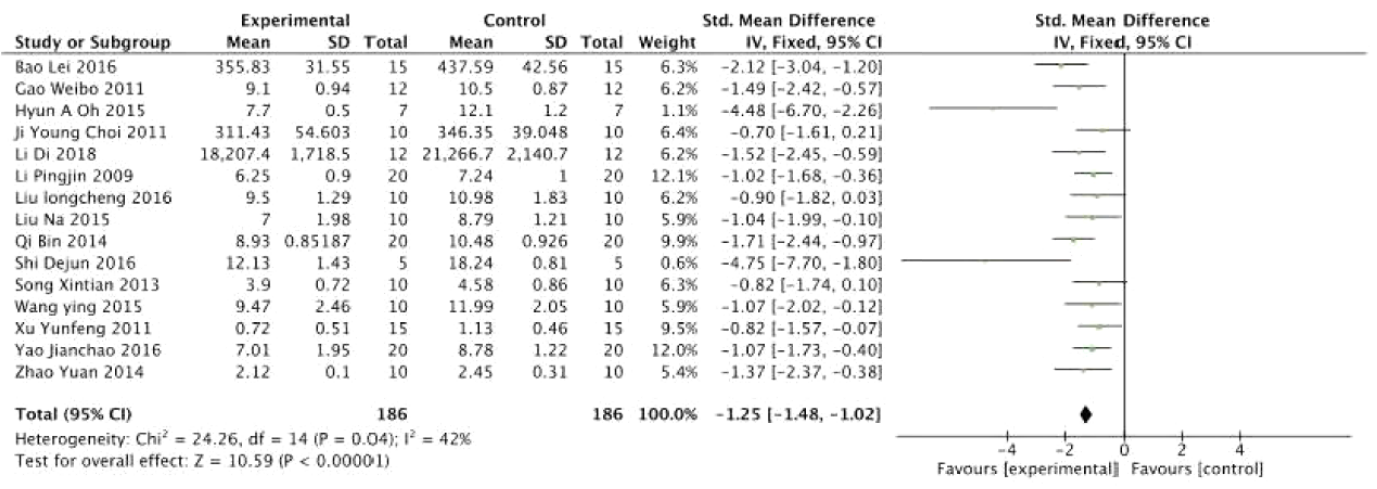

FIGURE 4 | (A) Level of glutathione peroxidase in serum of Panax ginseng C. A. Mey and its compounds versus Control. (B) Level of glucose in serum of Panax ginseng C. A. Mey and its compounds versus Control. (C) Level of blood urea nitrogen in serum of Panax ginseng C. A. Mey and its compounds versus Control. (D) Level of blood lactate in serum of Panax ginseng C. A. Mey and its compounds versus Control. 
A

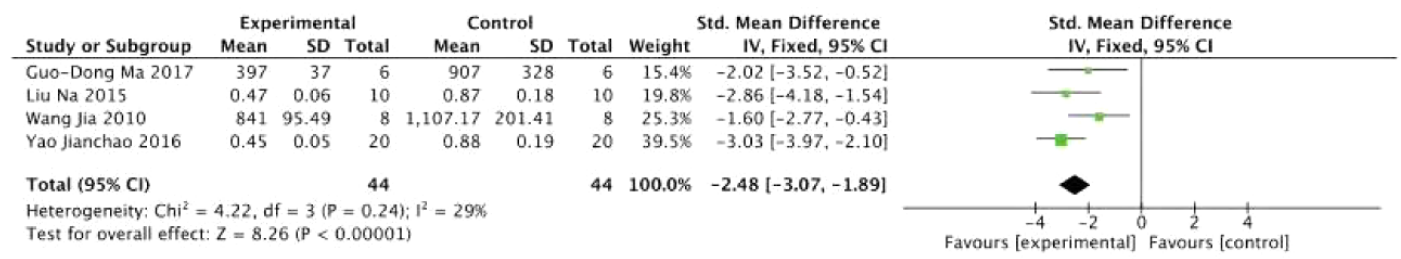

B

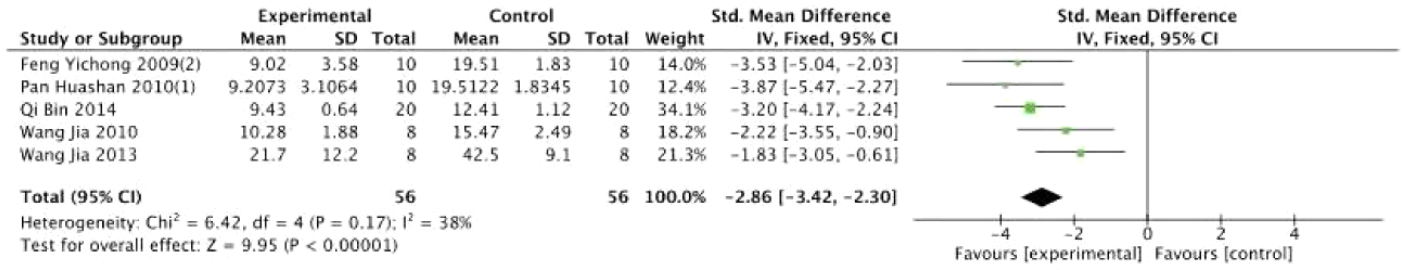

C

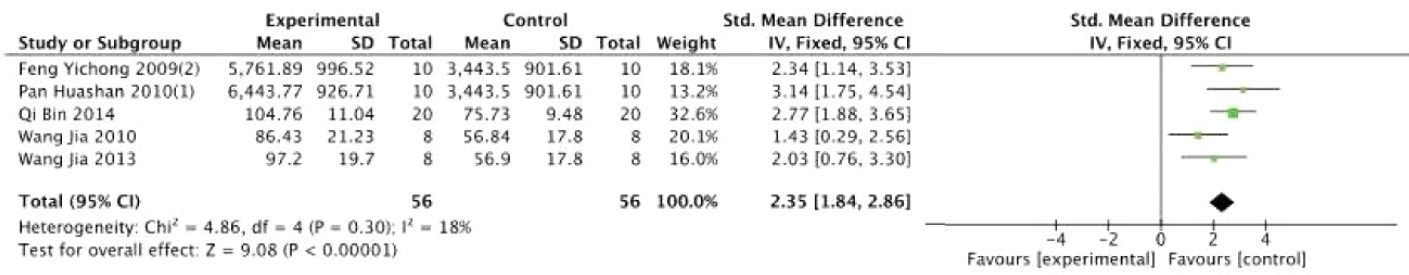

D

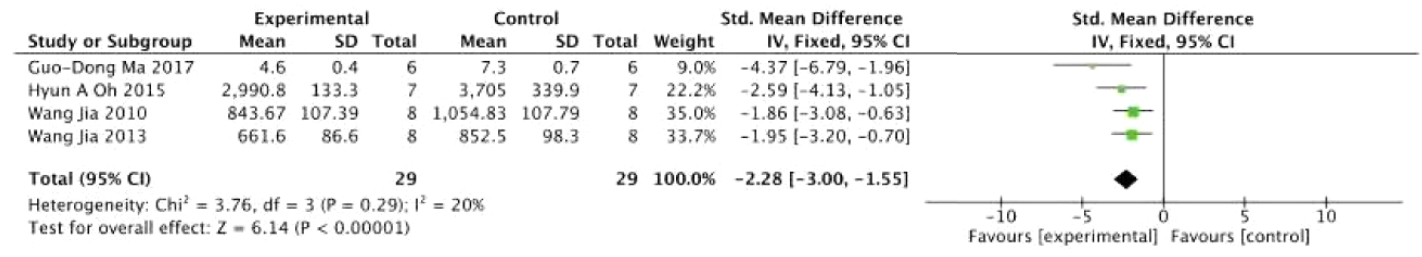

FIGURE 5 | (A) Level of creatine kinase in serum of Panax ginseng C. A. Mey and its compounds versus Control. (B) Level of malondialdehyde in serum of Panax ginseng C. A. Mey and its compounds versus Control. (C) Level of superoxide dismutase in serum of Panax ginseng C. A. Mey and its compounds versus Control. (D) Level of lactic dehydrogenase in serum of Panax ginseng C. A. Mey and its compounds versus Control.

PGC and control in all subgroups, including ginseng (Gao et al., 2011; Zhao et al., 2014; Shi et al., 2016; Ma et al., 2017), Ginsenosides (Liu et al., 2015; Wang et al., 2015; Yao, 2016), ginseng protein (Xu et al., 2011; Qi et al., 2014), and ginseng oligopeptides (Bao et al., 2016; Delgado et al., 2019) groups (Figure 7A). Meta-analysis of 4 studies (Feng et al., 2009c; Pan et al., 2010a; Liu et al., 2015; Yao, 2016) showed that PGC significantly decreased level of MDA compared with control $(\mathrm{n}=$ 50; $\mathrm{SMD}=-2.01 ; 95 \% \mathrm{CI},-2.50$ to $-1.51 ; P<000001$; heterogeneity: $\left.\chi^{2}=1.69, \mathrm{df}=3(P=0.64), \mathrm{I}^{2}=0 \%\right)$ (Figure
7B). Meta-analysis of two studies (Liu et al., 2015; Yao, 2016) showed no significant difference in activity of GSH-Px between PGC and control $(\mathrm{n}=30 ; \mathrm{SMD}=1.81 ; 95 \% \mathrm{CI},-7.98$ to 11.60 , $P=0.72$; heterogeneity: $\chi^{2}=0.00$, df $\left.=1(\mathrm{P}=1.00), \mathrm{I}^{2}=0 \%\right)$ (Figure 7C). Activity of SOD was reported in four studies (Feng et al., 2009c; Pan et al., 2010a; Liu et al., 2015; Yao, 2016). Two studies (Feng et al., 2009c; Pan et al., 2010a) showed significant difference $(P<0.05)$ for increasing activity of SOD compared PGC with control, whereas 2 studies (Liu et al., 2015; Yao, 2016) showed contradictory results $(P>0.05)$. 
A

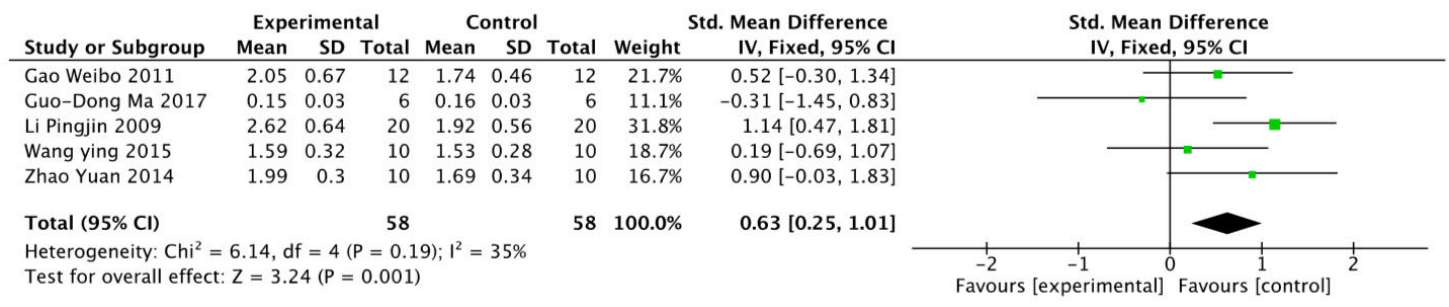

B

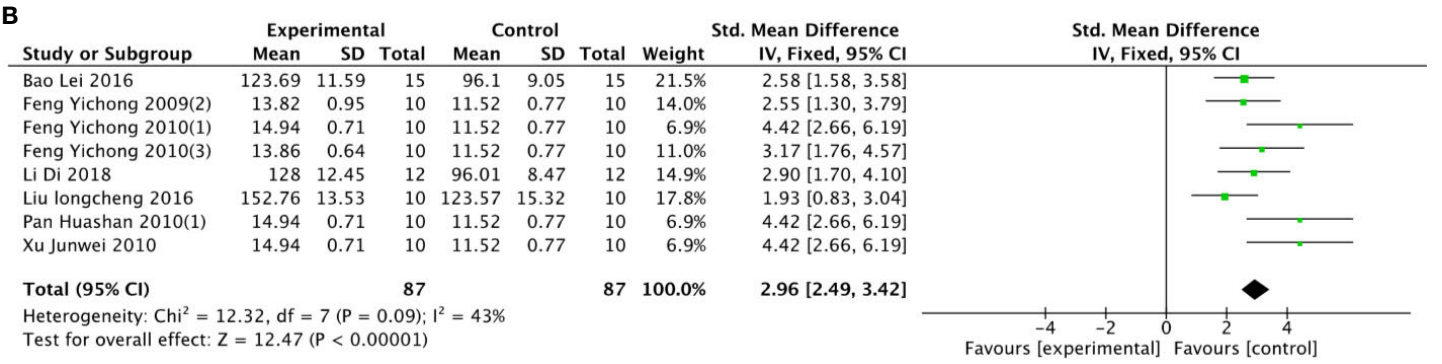

C

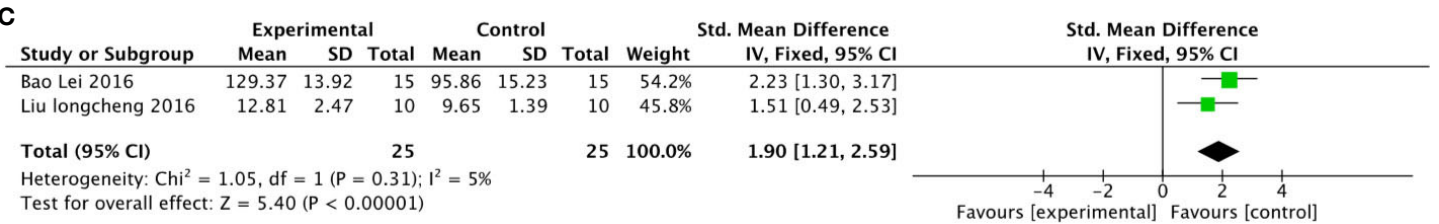

D

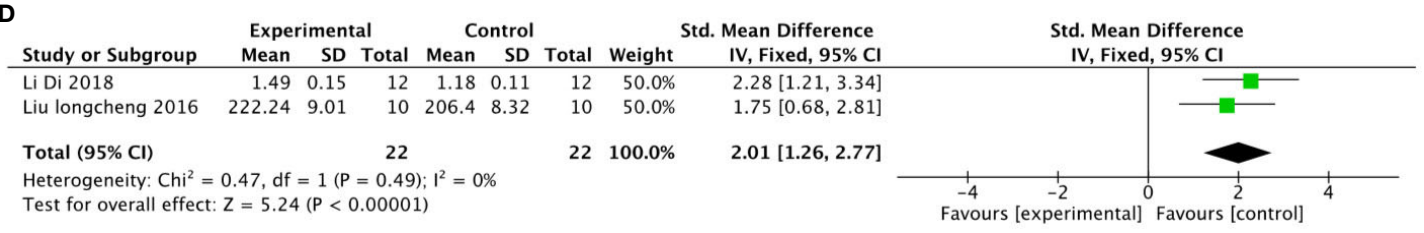

FIGURE 6 | (A) Level of glycogen in muscle of Panax ginseng C. A. Mey and its compounds versus Control. (B) Activity of superoxide dismutase in muscle of Panax ginseng C. A. Mey and its compounds versus Control. (C) Activity of catalase in muscle of Panax ginseng C. A. Mey and its compounds versus Control. (D) Activity of glutathione peroxidase in muscle of Panax ginseng C. A. Mey and its compounds versus Control.

\section{Brain Tissue Biochemical Parameters}

Meta-analysis of 5 studies (Feng et al., 2009a; Feng et al., 2009b; Pan et al., 2009; Feng et al., 2010a; Chen and Li, 2011) showed PGC was significant for reducing level of GABA ( $\mathrm{n}=55$; SMD = -4.45 ; $95 \% \mathrm{CI},-5.24$ to $-3.66 ; P<0.00001$; heterogeneity: $\chi^{2}=$ 17.97, $\left.\mathrm{df}=4(\mathrm{P}=0.001), \mathrm{I}^{2}=78 \%\right)$ and level of 5-HT $(\mathrm{n}=55$; SMD $=-1.19 ; 95 \% \mathrm{CI},-1.63$ to $-0.76 ; P<000001$; heterogeneity: $\chi^{2}=$ 15.52 , df $\left.=4(\mathrm{P}=0.004), \mathrm{I}^{2}=74 \%\right)$ compared with control. One study (Chen and $\mathrm{Li}, 2011$ ) that utilize chloral hydrate to anesthetize rats may be considered as the probable source of the heterogeneity. After getting rid of this study, there was showing that PGC better reduce the level of GABA $(\mathrm{n}=40$; SMD $=-6.12$; 95\% CI, -7.27 to $-4.96 ; P<000001$; heterogeneity: $\chi^{2}=3.10, \mathrm{df}=3$ $\left.(P=0.38), \mathrm{I}^{2}=3 \%\right)$ (Figure 8A) and 5-HT $(\mathrm{n}=40 ; \mathrm{SMD}=-1.77$; 95\% CI, -2.31 to -1.23 ; $P<0$ 00001; heterogeneity: $\chi^{2}=3.62, \mathrm{df}=$
$\left.3(P=0.31), I^{2}=17 \%\right)$ (Figure 8B). Meta-analysis of five studies (Feng et al., 2009a; Feng et al., 2009b; Pan et al., 2009; Feng et al., 2010a; Chen and Li, 2011) found significant difference for increasing level of Ach between PGC and control groups ( $\mathrm{n}=$ 55 ; $\mathrm{SMD}=1.86 ; 95 \% \mathrm{CI}, 1.38$ to $2.23 ; P<0$ 00001; heterogeneity: $\left.\chi^{2}=9.70, \mathrm{df}=4(\mathrm{P}=0.05), \mathrm{I}^{2}=59 \%\right)$. After removing one study (Feng et al., 2009a), meta-analysis of four studies (Feng et al., 2009b; Pan et al., 2009; Feng et al., 2010a; Chen and Li, 2011) showed similar results $(\mathrm{n}=45 ; \mathrm{SMD}=1.66 ; 95 \% \mathrm{CI}, 1.16$ to $2.16, P$ < 000001; heterogeneity: $\chi^{2}=3.21$, df $\left.=3(P=0.36), \mathrm{I}^{2}=6 \%\right)$ (Figure 8C). Meta-analysis of five studies (Feng et al., 2009a; Feng et al., 2009b; Pan et al., 2009; Feng et al., 2010a; Chen and Li, 2011) showed a significant increase in level of DA compared PGC with control ( $\mathrm{n}=55$; $\mathrm{SMD}=1.76$; $95 \% \mathrm{CI}, 1.26$ to 2.22 ; $P<000001$; heterogeneity: $\left.\chi^{2}=6.85, \mathrm{df}=4(\mathrm{P}=0.14), \mathrm{I}^{2}=42 \%\right)$ (Figure 8D). 
A

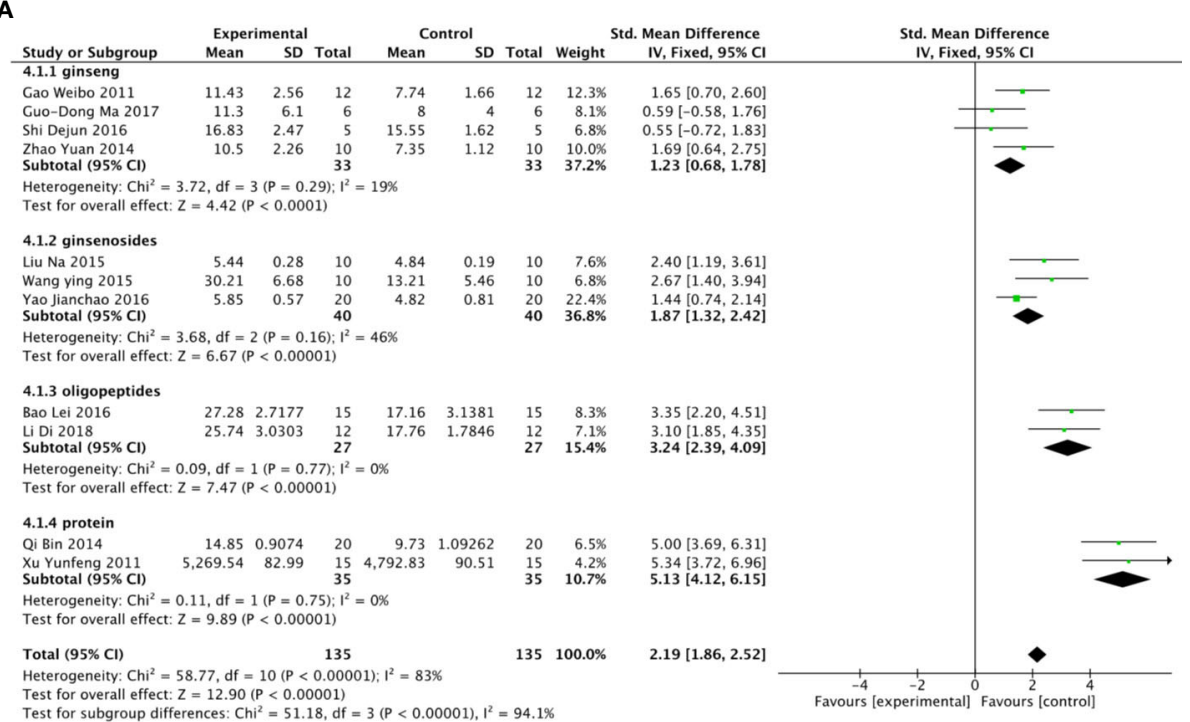

B

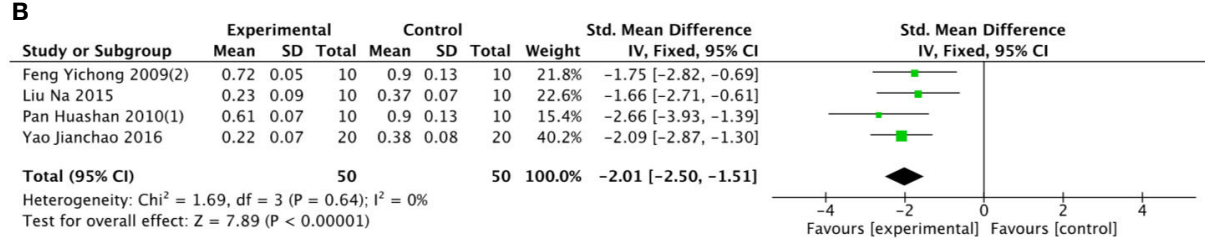

C

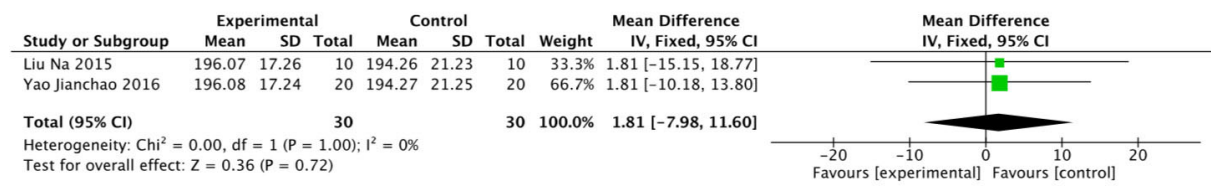

FIGURE 7 | (A) Level of glycogen in Liver of Panax ginseng C. A. Mey and its compounds versus Control. (B) Activity of malondialdehyde in Liver of Panax ginseng C. A. Mey and its compounds versus Control. (C) Activity of glutathione peroxidase in Liver of Panax ginseng C. A. Mey and its compounds versus Control.

\section{Animal Body Weight:}

Meta-analysis of 7 studies (Li et al., 2009; Xu et al., 2011; Song et al., 2013; Hwang et al., 2014; Bao et al., 2016; Liu, 2016; Shi et al., 2016) showed there was no significant difference in body weight between PGC and control groups ( $\mathrm{n}=96$; $\mathrm{SMD}=0.07$; 95\% CI: -0.21 to $0.36 ; P=0.62$; heterogeneity: $\chi^{2}=8.87, \mathrm{df}=6$ $\left.(\mathrm{P}=0.18), \mathrm{I}^{2}=32 \%\right)$ (Figure 9A).

\section{Publication Bias}

Funnel plots were conducted for two outcomes (Figures 9B, C). The results showed symmetrical distribution for the outcomes of BUN levels (Egger's test $\mathrm{t}=-1.05 ; 95 \% \mathrm{CI},-6.93$ to $2.50 ; P=$ 0.320 ), which did not suggest an obvious publication bias. However, there was a significant bias in the BLA levels with Egger's test $(t=-3.47 ; 95 \% \mathrm{CI},-5.14$ to $-1.19 ; P=0.004)$. Because the number of studies in the remaining outcomes was limited $(\mathrm{n}<10)$, funnel plot and Egger's test were not appropriate.

\section{Possible Mechanisms}

PGC improved activity of GSH-Px, CAT, and SOD, scavenged free radicals and their metabolites, reduced the excessive ROS, and decreased levels of MDA, CK, and LDH. PGC decreased nitric oxide synthase (NOS), reduced toxic oxidant peroxynitrite, and prevented mitochondrial dysfunction and lipid peroxidation. PGC may enhance fat mobilization and promote gluconeogenesis, increase the delivery of glucose, and maintain blood glucose level. PGC increased the LDH activity and the hepatic glycogen levels, and retarded the accumulation of BUN and BLA. PGC improved succinate dehydrogenase (SDH), $\mathrm{Na}^{+}-\mathrm{K}^{+}$-ATPase, and $\mathrm{Ca}^{2+} \mathrm{Mg}^{2+}$-ATPase activities, enhanced mitochondrial function, and produced more adenosine triphosphate (ATP). PGC attenuated $\mathrm{MPP}^{+}$-induced MPTP-induced and apoptosis. PGC increased Ach and DA levels, and decreased GABA and 5-HT levels in brain tissue (Figure 10).

\section{DISCUSSION}

\section{Summary of Evidence}

This is the clinical and preclinical systematic review to evaluate the efficacy and safety of PGC for fatigue. Eight RCTs with 678 
A

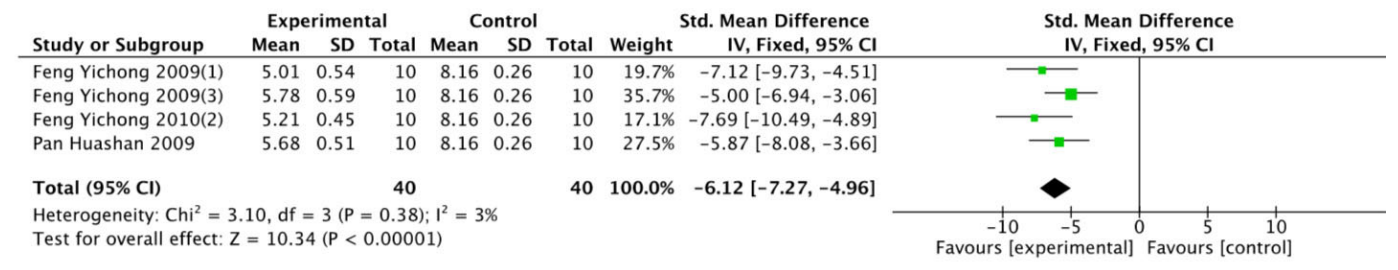

B

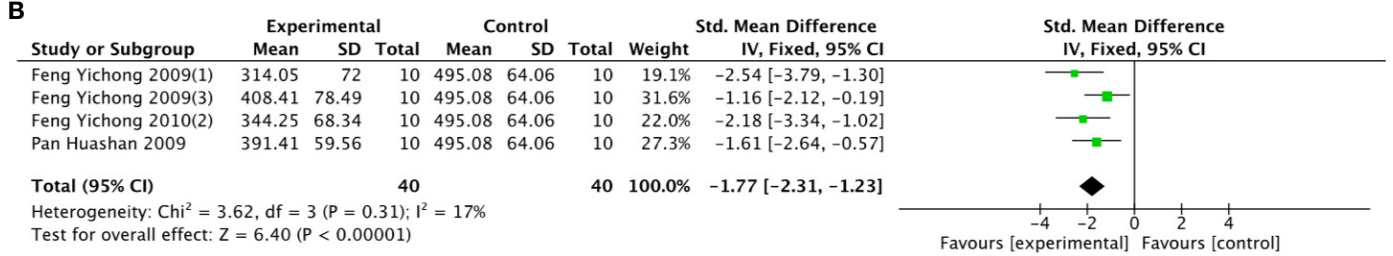

C

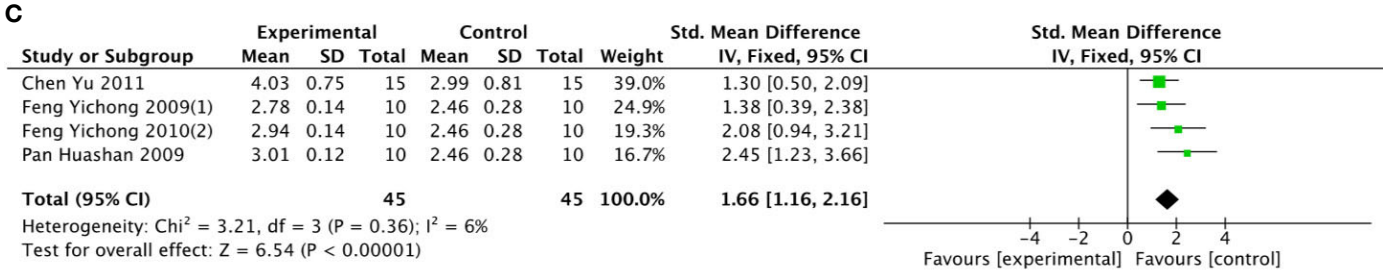

D

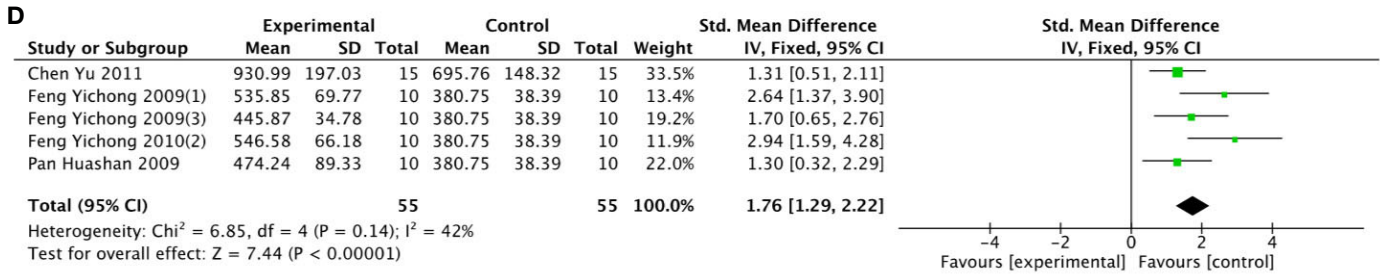

FIGURE 8 | (A) Level of gamma-aminobutyric acid in brain tissue of Panax ginseng C. A. Mey and its compounds versus Control. (B) Level of 5-hydroxytryptamine in brain tissue of Panax ginseng C. A. Mey and its compounds versus Control. (C) Level of acetylcholine in brain tissue of Panax ginseng C. A. Mey and its compounds versus Control. (D) Level of dopamine in brain tissue of Panax ginseng C. A. Mey and its compounds versus Control.

participants and 30 studies with 2249 animals were selected. The quality of RCTs included was high, and animal studies were generally moderate. The findings of RCTs demonstrated that PGC was superior to placebo according to their respective fatigue scales, heart rate recovery, and clinical effect. There were a similar number of adverse effects between PGC and placebo group. The evidence available from animal studies showed that PGC could preserve physical function after exercise, mainly through antioxidant, regulating carbohydrate metabolism, delaying the accumulation of metabolites, promoting mitochondrial function, neuroprotection, antiapoptosis, and regulating neurotransmitter disorder in central nervous system.

\section{Limitations}

Some methodological flaws existed in the primary RCTs. First of all, 3 studies used allocation concealment (Hartz et al., 2004; Hyeong-Geug et al., 2013; Lee et al., 2016). Trials with unreported or inadequate allocation concealment could be exaggerated an average $18 \%$ beneficial effect of interventions
(Higgins and Green, 2011). Second, non-blinding of outcome assessment may lead to systemic errors. In previous reviews, nonblinding of outcome assessment can lead to an overestimation of treatment effect by $27 \%$ to $68 \%$, depending on different outcome types, i.e., binary outcome, measurement scale outcome, and time-to-event outcome. However, in RCTs, blinded outcome assessment was commonly poor reported and used. In present study, only 2 RCTs (Hartz et al., 2004; Lee et al., 2016) reported blinding of outcome assessment. The observer bias can be avoided by sufficient blinding. More independent assessors can be further used to increase the feasibility of blind assessment (Brennan et al., 2015). Thirdly, 3 RCTs (Hyeong-Geug et al., 2013; Kim et al., 2016; Lee et al., 2016) formally registered. Clinical trial registration could help to minimize bias in selective reporting and improve the validity and value of the scientific evidence (Angelis et al., 2006). Fourthly, intention to treat (ITT) analysis is a strategy to gather data as completely as possible on all randomized patients in line with their scheduled assessments (Lewis and Machin, 1993). Four RCTs (Hermann-J et al., 2003; 
A

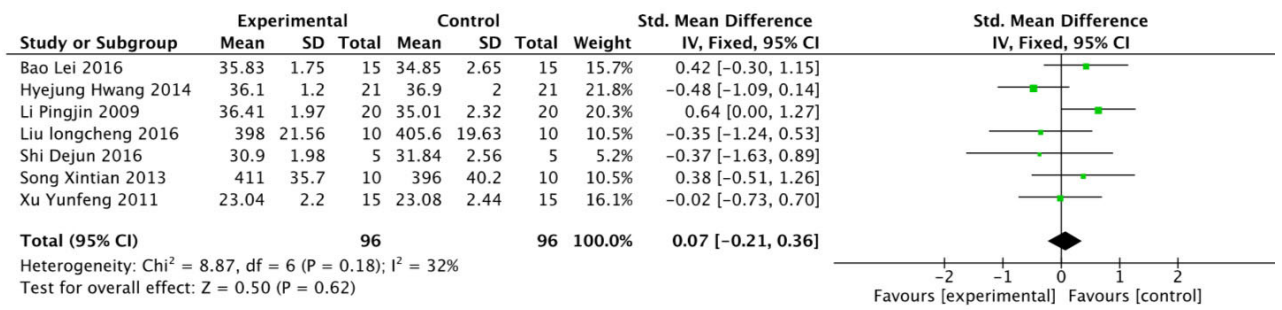

B

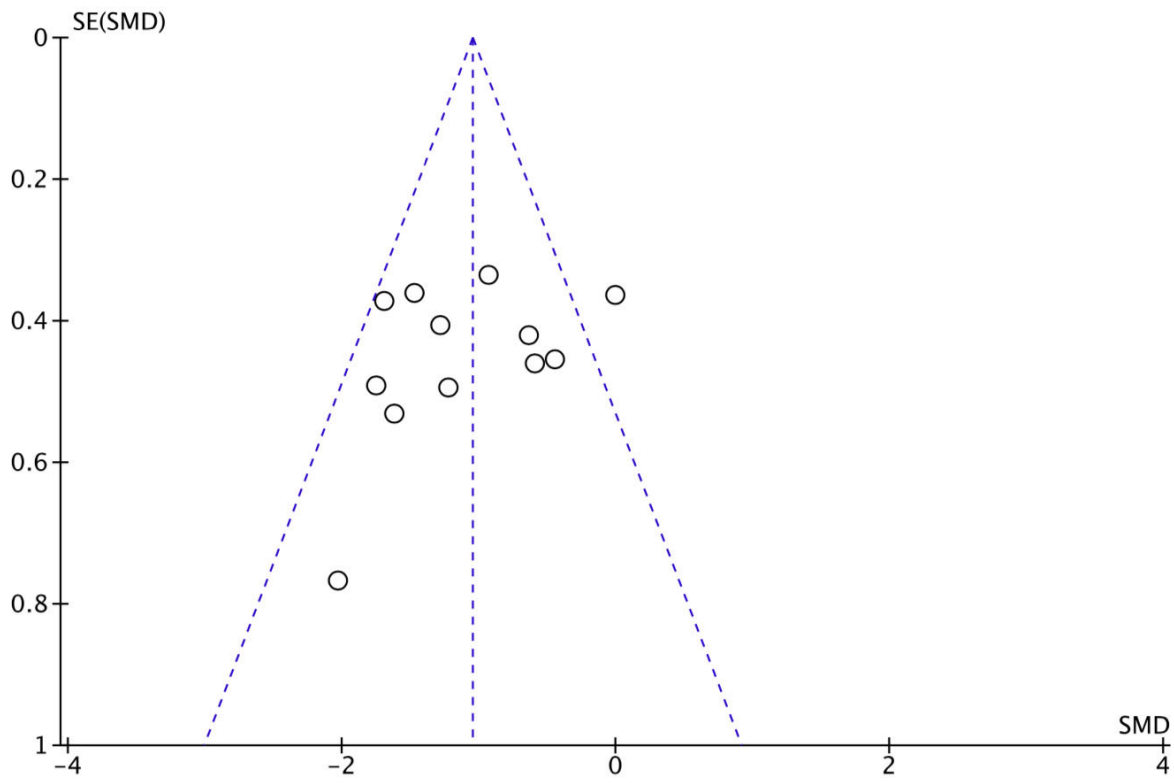

C

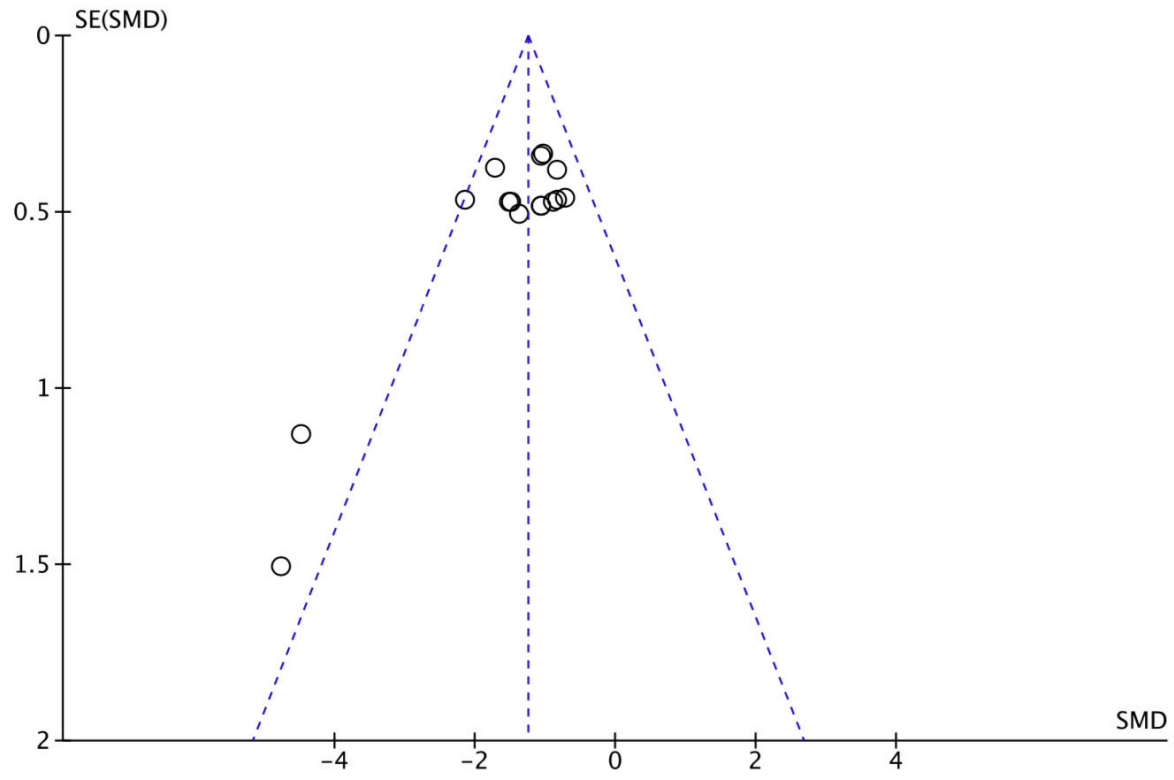

FIGURE 9 | (A) Body weight of Panax ginseng C. A. Mey and its compounds versus Control. (B) Funnel plots of blood urea nitrogen. (C) Funnel plots of blood lactate. 


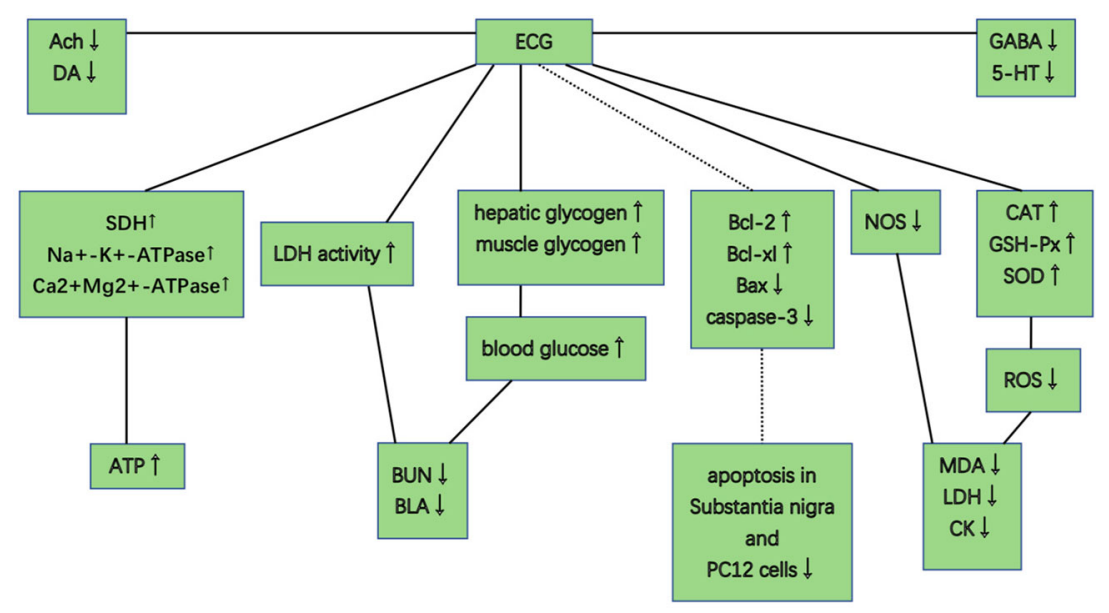

FIGURE 10 | Possible mechanisms of Panax ginseng C. A. Mey and its compounds anti-fatigue.

Hyeong-Geug et al., 2013; Kim et al., 2016; Lee et al., 2016) described whether they analyzed the data based on the ITT principle. Trials with incorrect or no ITT analysis may overestimate the results (Bondemark and Abdulraheem, 2017). Finally, various scales of fatigue were used as outcome measure in different trials. The evaluation of clinical efficacy rate is based on inconsistent scales, which restrict the validity and reliability. The quality of animal studies was considered to be moderate, suggesting that the results should be carefully interpreted. Fatigue can be divided into CFS and post-exercise fatigue in clinic. In the present analysis, all fatigue models are exhaustion, which may lead to ignore chronic process.

In addition, we cannot neglect the contribution of Korea and other countries worldwide in the study of ginseng in treatment of fatigue. Due to the limitation of language, the present study was not included studies which language was not English or Chinese. We can increase international cooperation to overcome the linguistic limitation.

\section{Implications}

The evidence available from present study supported the routine use of PGC for fatigue, whereas the safety still needs more data because only five of eight studies reported. Given the gap between limitations of the primary RCTs and the quality of RCTs, we recommend that further design of the RCTs should consult the CONSORT statement (Moher et al., 2005), which offer a standard way for authors to design, conduct, analyze, and interpret, and to assess the validity of results.

Currently, there is no gold standard for measuring fatigue. Various scales were used to measure fatigue in different studies. Some scales have been calibrated, and some are homemade. Measurement of fatigue is challenging. Due to the wide range of conceptualizations of the problem and the concurrent development of questionnaires for many specific diseases, many questionnaires are used to measure fatigue. A comprehensive fatigue measurement, such as the Fatigue Severity Scale (Krupp et al., 1989), Piper Fatigue Scale (Piper et al., 1998), or FACIT-F
(David et al., 2010), assesses the impact of fatigue on daily activities and its severity. In addition, short fatigue measurements such as the POMS-B fatigue subscale (Mcnairpm and Dropplemannl, 1992) and the 7-item Patient Reported Outcome Measurement Information System Cancer Fatigue Short Form (Cessna et al., 2016; Garcia et al., 2016) mainly assess severity of fatigue. The fatigue measurement precision with a comprehensive measure was greater than that with short fatigue measurement in evaluating moderate to severe fatigue, whereas the short fatigue measurement performed better in evaluating mild fatigue (Voshaar et al., 2015). Therefore, we should select the suitable fatigue measurements based on the research requirements.

Degree and scope of debilitating fatigue is a core component of health care where chronic diseases are receiving increasing attention. Current acute disease research models are not enough to solve chronic disorders affecting multiple regulatory systems and present complex constellations of symptoms. The identification of objective markers consistently associated with CFS is an important goal in relation to diagnosis and treatment, as the current case definitions are based entirely on physical signs and symptoms. Since the human body is an autonomous, fully integrated, and self-regulating system, it is not surprising that even localized muscle fatigue can present systemic biomarkers. There is a growing study devoted to understanding the biology of fatigue. Recognition of CFS biomarkers is an important part of this work. A complex construct of symptoms emerges from alterations and/ or dysfunctions in the nervous, endocrine, and immune systems. Biomarkers, distributing across these systems, constituted complex biological networks. The acquisition of biomarkers required a comprehensive biological network-based analysis of fatigue biology (Klimas et al., 2012). In addition, molecular aberrations observed in many CFS blood cell studies provided an opportunity to develop diagnostic analysis of blood samples. With the development of micro/nanofabrication, direct electrical detection of cellular and molecular properties, microfluidics, and artificial intelligence techniques, a nano-electronics blood-based assay have been developed, which can potentially establish 
diagnostic biomarkers and drug screening platform for CFS (Esfandyarpour et al., 2019).

Preclinical research is the key to convert preclinical data into clinical data (Ramirez et al., 2017). However, there is growing concern that poor experimental design and transparent reporting lead to frequent failure of translating preclinical discoveries into new therapies for human diseases (Hackam and Redelmeier, 2006). In present study, the quality of including animal studies was moderate. We recommend that further design of the studies should consult the ARRIVE guidelines (Kilkenny et al., 2012) and use appropriate animals, random allocation, model blinded induction, and outcomes blinded assessment to improve the accuracy of the results.

PGC acted through complex, multicompound, multitarget, and multipathway mechanisms in fatigue and might prove to be of great value in further clinical trials. The possible mechanisms of PGC for fatigue are summarized as follows: (1) Antioxidant stress: PGC passed through the injured membrane, improved the activity of GSH-Px, CAT, and SOD, scavenged hydroxyl radical, and reduced the excessive ROS, and thus preventing lipid oxidation and protecting the corpuscular membrane to reduce the release of $\mathrm{LDH}, \mathrm{MDA}$, and CK into the serum (Wang et al., 2014; Zheng et al., 2017). Another mechanism might involve the nitric oxide pathway. NOS, a pro-oxidative enzyme, increased the production of toxic oxidant peroxynitrite. PGC decreased NOS and prevented peroxynitrite-induced mitochondrial dysfunction and lipid peroxidation (Ki Sung et al., 2006); (2) Regulation of carbohydrate metabolism: PGC increased the proportion of energy supplied by fat and promoted gluconeogenesis to improve hepatic glycogen storage. PGC enhanced the delivery of glucose by increasing capillary perfusion and plasma glucose concentration and increased the permeability of the muscle membrane of glucose to increase the muscle glucose uptake during exercise (Wang et al., 2015; Ma et al., 2017). A reduced rate of hepatic and muscle glycogen break-down and a greater potential for fatty acid metabolism could maintain blood glucose level, and thus enhancing exercise capacity (Favier and Koubi, 1988); (3) Delaying the accumulation of metabolites: With the accumulation of BUN and BLA, the $\mathrm{pH}$ in muscle tissue and blood reduced, which could obstruct the transmission of excitation at neuromuscular junctions, reduce the maximum tension and sustainability of muscle tissue, and hinder the process of sugar supply. PGC increased the LDH activity and the glycogen levels and retarded the accumulation of BUN and BLA (Oh et al., 2015; Delgado et al., 2019); (4) Promotion of mitochondrial function: $\mathrm{SDH}$ is a key enzyme associated with the regulation of the tricarboxylic acid cycle, catalyzing the synthesis of ATP. In addition, $\mathrm{Na}^{+}-\mathrm{K}^{+}$-ATPase and $\mathrm{Ca}^{2+}-\mathrm{Mg}^{2+}$-ATPase are crucial enzymes to degrade ATP. NRF-1 and TFAM are positive regulators of transcription. PGC improved $\mathrm{SDH}, \mathrm{Na}^{+}-\mathrm{K}^{+}-$ ATPase, and $\mathrm{Ca}^{2+} \mathrm{Mg}^{2+}$-ATPase activities. PGC enhanced the mRNA expression of NRF-1 and TFAM and increased the mtDNA content, and thereby enhancing mitochondrial function and producing more ATP for energy supplementation (Delgado et al., 2019); (5) Neuroprotection and antiapoptosis: PGC had a protective effect against MPTP-induced apoptosis in the mouse substantial nigra. This anti-apoptotic effect of PGC may be attributed to enhanced expression of Bcl-2 and Bcl-xl, reduced expression of bax and nitric oxide synthase, and inhibited activation of caspase-3 (Radad et al., 2006); (6) Regulation of neurotransmitter disorder: PGC decreased GABA and 5-HT levels, thereby increasing central nervous system excitability. PGC decreased the activity of acetylcholinesterase, maintained normal Ach and norepinephrine levels in cholinergic neurons, and enhanced the level of DA in the hippocampus (Feng et al., 2009b; Chen and Li, 2011).

\section{CONCLUSIONS}

The findings of present study demonstrated that PGC exerted antifatigue function, mainly through antioxidant stress, regulation of carbohydrate metabolism, delaying the accumulation of metabolites, promotion of mitochondrial function, neuroprotection and antiapoptosis, and regulation of neurotransmitter disorder. And the findings supported, at least to an extent, the use of PGC for fatigue.

\section{AUTHOR CONTRIBUTIONS}

Study conception and design: YL/G-QZ. Acquisition, analysis and/or interpretation of data: T-YJ/P-QR/H-YL/P-PZ/G-QZ/YL Final approval and overall responsibility for this published work: YL/G-QZ.

\section{FUNDING}

This study was supported by a grant from the National Natural Science Foundation of China (81973657/H2902); priority speciality of integrative brain diseases, the second affiliated hospital of Wenzhou Medical University, Wenzhou China (2016).

\section{REFERENCES}

Alraek, T., Lee Ms Fau - Choi, T.-Y., Choi Ty Fau - Cao, H., Cao H Fau - Liu, J., and Liu, J. (2011). Complementary and alternative medicine for patients with chronic fatigue syndrome: a systematic review. Complementary Altern. Med. 11, 87-98. doi: 10.1186/1472-6882-11-87

Angelis, C. D., De Drazen, J. M., Frizelle, F. A., Charlotte, H., John, H., Richard, H., et al. (2006). Is this clinical trial fully registered? A statement from the

International Committee of Medical Journal Editors. Am. J. Ophthalmol. 140, 567-567. doi: 10.1016/j.ajo.2005.07.004

Arring, N. M., Millstine, D., Marks, L. A., and Nail, L. M. (2018). Ginseng as a Treatment for Fatigue: A Systematic Review. J. Altern. Complementary Med. 24, acm.2017.0361. doi: 10.1089/acm.2017.0361

Bach, H. V., Kim, J., Myung, S. K., and Cho, Y. A. (2016). Efficacy of Ginseng Supplements on Fatigue and Physical Performance: a Meta-analysis. J. Korean Med. Sci. 31, 1879-1886. doi: 10.3346/jkms.2016.31.12.1879 
Bao, L., Cai, X., Wang, J., Zhang, Y., Sun, B., and Li, Y. (2016). Anti-Fatigue Effects of Small Molecule Oligopeptides Isolated from Panax ginseng C. A. Meyer in Mice. Nutrients, 8, 807-818. (Electronic). doi: 10.3390/nu8120807

Bondemark, L., and Abdulraheem, S. (2017). Intention to treat (ITT) analysis as reported in orthodontic randomized controlled trials-evaluations of methodology and recommendations for the accurate use of ITT analysis and handling dropouts. Eur. J. Orthodontics 40, 409-413. doi: 10.1093/ejo/cjx084

Brennan, C. K., Sunita, R., and Suzie, R. (2015). Blinded Outcome Assessment WasInfrequently Used and Poorly Reported in Open Trials. PLOS ONE 10 (6), e01. doi: 10.1371/journal.pone.0131926

Cessna, J. M., Jim, H. S., Sutton, S. K., Asvat, Y., Small, B. J., Salsman, J. M., et al. (2016). Evaluation of the psychometric properties of the PROMIS Cancer Fatigue Short Form with cancer patients. J. Psychosomatic Res. 81, 9-13. doi: 10.1016/j.jpsychores.2015.12.002

Chen, Y., and Li, N. (2011). Effects of Total Saponins of Panax Ginseng on Contents of Monoamine Transmitter in Hippocampal Tissue of Rats Exposed with Exercise Fatigue. J. Xi'an Institute Phys. Educ. 28, 99-101+128. doi: 10.16063/j.cnki.issn1001-747x.2011.01.010

Chen, M. K. (1986). The epidemiology of self-perceived fatigue among adults. Preventive Med. 15, 74-81. doi: 10.1016/0091-7435(86)90037-X

Choi, J. Y., Woo, T. S., Yoon, S. Y., Campomayor Dela, P. I., Choi, Y. J., Choi Yj Fau - Ahn, H. S., et al. (2011). Red ginseng supplementation more effectively alleviates psychological than physical fatigue. J. Ginseng Res. 35, 331-338. doi: 10.5142/jgr.2011.35.3.331

Collatz, A., Johnston, S. C., Staines, D. R., and Marshall-Gradisnik, S. M. (2016). A Systematic Review of Drug Therapies for Chronic Fatigue Syndrome/Myalgic Encephalomyelitis. Clin. Ther. 38, 1263-1271.e1269. doi: 10.1016/ j.clinthera.2016.04.038

David, C., Jin-Shei, L., Chih-Hung, C., Amy, P., and Mitchell, S. (2010). Fatigue in cancer patients compared with fatigue in the general United States population. Cancer 94, 528-538. doi: 10.1002/cncr.10245

Delgado, A., Oliveira, P., Goes, P. S. A., and Lemos, A. (2019). Development and analysis of measurement properties of the "maternal perception of childbirth fatigue questionnaire" (MCFQ). Braz. J. Phys. Ther. 23, 2-13. doi: 10.1016/ j.bjpt.2019.01.004

Engels, H. J., Ed, J. M. S. M., and John, C. W. (1996). Failure of chronic ginseng supplementation to affect work performance and energy metabolism in healthy adult females. Nutr. Res. 16, 1295-1305. doi: 10.1016/0271-5317(96)00138-8

Engels, H. J., Fahlman, M .M., and Wirth, J. C. (2003). Effects of ginseng on secretory IgA, performance, and recovery from interval exercise. Med. Sci. Sports Exerc. 35, 690-696. doi: 10.1249/01.MSS.0000058363.23986.D2

Engels, H. J., Kolokouri, I., Nd, C. T., and Wirth, J. C. (2001). Effects of ginseng supplementation on supramaximal exercise performance and short-term recovery. J. Strength Condition. Res. 15, 290. doi: 10.1249/01.MSS.0000058 36323986.D2

Esfandyarpour, R., Kashi, A., Nemat-Gorgani, M., Wilhelmy, J., and Davis, R. W. (2019). A nanoelectronics-blood-based diagnostic biomarker for myalgic encephalomyelitis/chronic fatigue syndrome (ME/CFS). Immunol. Infalmm. 116, 21-29. doi: 10.1073/pnas.1901274116

Favier, R. J., and Koubi, H. E. (1988). Metabolic and structural adaptations to exercise in chronic intermittent fasted rats. Am. Physiol. Soc. 254, 877-884. doi: 10.1152/ajpregu.1988.254.6.R877

Feng, C. Y., Pan, H. S., Zhao, Z. M., and Li, J. H. (2009a). Experimental Study on the Changes of Central Neural Transmitters and the Anti-Fatigue Effects of Ginsenoside Rb1,Re in Rats with Kinesigenic Fatigue. Hubei J. Tradit. Chin. Med. 31, 5-7. doi: 10.3969/j.issn.1000-0704.2009.03.002

Feng, C. Y., Pan, H. S., Zhao, Z. M., and Yang, C. X. (2009b). Effects of Ginsenoside Re and Ginseng Total Saponin on Hypothalamic Ach,DA,5-HT and GABA in Exercise-induced Fatigue Rats. Fujian J. Tradit. Chin. Med. 40, 44-46. doi: 10.3969/j.issn.1000-338X.2009.02.023

Feng, C. Y., Zhao, Z. M., Chen, Y., HS, P., JH, Li, BG, B., et al. (2009c). Effects of Ginsenoside Re on MDA Content and SOD Activity in Rats with Exercise induced Fatigue. Tradit. Chin. Drug Res. Clin. Pharmacol. 20, 542-544. doi: 10.13260/j.cnki.jftcm.009687

Feng, C. Y., Pan, H. S., Bian, B. G., Zhao, Z. M., and Chen, C. J. (2010a). Experimental Studies on Resisting Exercise-induced central Fatigue of Ginsenoside Rg1 and Rb1. J. Pla. Institute Phys. Educ. 29, 112-115. doi: 10.3969/j.issn.1671-1300.2010.03.033
Feng, C. Y., Pan, H. S., Zhao, Z. M., and Wen, X. (2010b). The Effects of Ginsenoside Rb1 and Ginsenosides on the Biochemical Index of Rat Skeletal Muscle with Exercise-induced Fatigue. J. Nanjing Institute Phys. Educ. (Natural Sci.) 9, 24-25+44. doi: 10.15877/j.cnki.nsin.2010.01.002

Feng, C. Y., Xu, Z. W., Pan, H. S., and Zhao, Z. M. (2010c). Effects of Ginsenoside Rgl on Structure and Function of Rat Skeletal Muscle with Exercise-induced Fatigue. J. Guangzhou Univ. Tradit. Chin. Med. 27, 40-44. doi: 10.13359/ j.cnki.gzxbtcm.2010.01.018

Fukuda, K., Straus SE,, Hickie, I., Sharpe, M. C., Dobbins, J. G., and Komaroff, A. (1994). The chronic fatigue syndrome: a comprehensive approach to its definition and study. Int. Chronic Fatigue Syndrome Study Group 121, 953959. doi: 10.7326/0003-4819-121-12-199412150-00009

Gal, M. L., Cathebras, P., and Strüby, K. (1996). Pharmaton capsules in the treatment of functional fatigue: A double-blind study versus placebo evaluated by a new methodology. Phytother. Res. 10, 49-53. doi: 10.1002/(SICI)10991573(199602)10:1<49::AID-PTR772>3.0.CO;2-M

Gao, W. B., Mi, J., Qin, Q. J., Sun, J. H., Wu, W., Wang, S. M., et al. (2011). Antifatigue Effects of Ginseng and Its Processed Products. Chin. J. Exp. Tradit. Med. Formul. 17, 210-213. doi: 10.13422/j.cnki.syfjx.2011.19.071

Garcia, S. F., David, C., Clauser, S. B., Flynn, K. E., Thomas, L., Jin-Shei, L., et al. (2016). Standardizing patient-reported outcomes assessment in cancer clinical trials: a patient-reported outcomes measurement information system initiative. J. Clin. Oncol. 25, 5106-5112. doi: 10.1200/JCO.2007.12.2341

Hackam, D. G., and Redelmeier, D. A. (2006). Translation of research evidence from animals to humans. JAMA J. Am. Med. Assoc. 296, 1731-1732. doi: 10.1001/jama.296.14.1731

Hartz, A. J., Bentler, S., Noyes, R., Hoehns, J., Logemann, C., Sinift, S., et al. (2004). Randomized controlled trial of Siberian ginseng for chronic fatigue. psychol. Med. 34, 51-61. doi: 10.1017/S0033291703008791

Higgins, J. P., and Green, S. (2011). Cochrane Handbook for Systematic Reviews of Interventions. Naunyn-Schmiedebergs Archiv. Für Experiment. Pathol. Und Pharmakol. 5, S38.

Hwang, H., Kim, J., Park, J., Yun, H., Cheon, W. K., Kim, B., et al. (2014). Red ginseng treatment for two weeks promotes fat metabolism during exercise in mice. Nutrients, 1874-1885. doi: 10.3390/nu6051874

Hyeong-Geug, K., Jung-Hyo, C., Sa-Ra, Y., Jin-Seok, L., Jong-Min, H., Nam-Hun, L., et al. (2013). Antifatigue effects of Panax ginseng C.A. Meyer: a randomised, double-blind, placebo-controlled trial. PloS One 8, e61271. doi: 10.1371/ journal.pone.0061271

Jason, L. A., Evans, M., Brown, M., and Porter, N. (2010). What is fatigue? Pathological and nonpathological fatigue. Am. Acad. Phys. Med. Rehabil., 2, 327-331. doi: 10.1016/j.pmrj.2010.03.028

Jones, J. F., Maloney, E. M., Boneva, R. S., Boneva, R. S., Jones, A. B., and Reeves, W. C. (2007). Complementary and alternative medical therapy utilization by people with chronic fatiguing illnesses in the United States. Complement. Altern. Med., 7, 12-22. doi: 10.1186/1472-6882-7-12

Kahan, B. C., Rehal, S., and Cro, S. (2015). Blinded Outcome Assessment Was Infrequently Used and Poorly Reported in Open Trials. PloS One 10 (6), e0131926. doi: 10.1371/journal.pone.0131926

Ki Sung, K., Takako, Y., Hyun Young, K., and Jeong Hill, P. (2006). Study on the nitric oxide scavenging effects of ginseng and its compounds. J. Agric. Food Chem. 54, 2558. doi: 10.1021/jf0529520

Kilkenny, C., Browne, W., Cuthill, I., Emerson, M., and Altman, D. (2012). Improving bioscience research reporting: The ARRIVE guidelines for reporting animal research. Veterinary Clin. Pathol. 41, 27-31. doi: 10.1111/ j.1939-165X.2012.00418.x

Kim, S. N., Ha, Y. W., Shin, H., Son, S. H., Wu, S. J., and Kim, Y. S. (2007). Simultaneous quantification of 14 ginsenosides in Panax ginseng C.A. Meyer (Korean red ginseng) by HPLC-ELSD and its application to quality control. J. Pharmaceut. Biomed. Anal. 45, 164-170. doi: 10.1016/j.jpba.2007.05.001

Kim, K. M., Kim, M. J., Song, S. W., Cho, D. Y., Park, K. C., Yang, S. W., et al. (2016). Efficacy and Safety of "URSA Complex" in Subjects with Physical Fatigue: A Multicenter, Randomized, Double-blind,Placebo-controlled Trial. Chin. Med. J. 129, 129-134. doi: 10.4103/0366-6999.173432

Klimas, N. G., Broderick, G., and Fletcher, M. A. J. (2012). Biomarkers for chronic fatigue. Brain. Behav. Immun. 26. 1202-1210. doi: 10.1002/nau.24423

Krupp, L. B., Larocca, N. G., Muir-Nash, J., and Steinberg, A. D. (1989). The fatigue severity scale. Application to patients with multiple sclerosis and 
systemic lupus erythematosus. Arch. Neurol. 46, 1121-1123. doi: 10.1001/ archneur.1989.00520460115022

Lee, N., Lee, S. H., Yoo, H. R., and Yoo, H. S. (2016). Anti-Fatigue Effects of Enzyme-Modified Ginseng Extract: A Randomized, Double-Blind, PlaceboControlled Trial. J. Altern. Complement Med. 22, acm.2016.0057. doi: 10.1089/ acm.2016.0057

Lewis, J. A., and Machin, D. (1993). Intention to treat-who should use ITT? Br. J. Cancer 68, 647-650. doi: 10.1038/bjc.1993.402

Lewis, G., and Wessely, S. (1992). The epidemiology of fatigue: more questions than answers. J. Epidemiol. Community Health 46, 92-97. doi: 10.1136/ jech.46.2.92

Li, D., Ren, J. W., Zhang, T., Liu, R., Wu, L., Dua, Q., et al (2018). Anti-fatigue effects ofsmall-molecule oligopeptides isolated from Panax quinquefolium L. in mice. Food Funct, 9, 8-16. doi: 10.1039/c7fo01658a

Li, P. J., Zhang, T., Wang, X. Y., and Wang, F. (2009). The study of anti-fatigue action of pseudo-ginsenoside GQ on exhaustive mouses. Chin. J. Lab. Diagn. 13, 869-871. doi: 10.3969/j.issn.1007-4287.2009.07.006

Liu, N., Liu, K., Liu, B. C., Wu, X. Q., Ding, Y., Xie, W., et al. (2015). Study on Antifatigue Effect of Ginsenoside on Mice. Guangming J. Chin. Med. 30, 1867-1869. doi: $10.3969 /$ j.issn.1003-8914.2015.09.022

Liu, L. C. (2016). Ginsenoside Rg1 has Impacts Exhaustive Exercise Rats of Skeletal Muscle and Brain Tissue Carbonylated Protein Content[Master"s] (Hunan Normal University). Available at: http://cdmd.cnki.com.cn/Article/CDMD10542-1016085811.htm.

Ma, G. D., Chiu, C. H., Hsu, Y. J., Hou, C. W., Chen, Y. M., and Huang, C. C. (2017). Changbai Mountain Ginseng (Panax ginseng C.A. Mey) Extract Supplementation Improves Exercise Performance and Energy Utilization and Decreases Fatigue-Associated Parameters in Mice.LID - E237 [pii] LID 10.3390/Molecules 22020237.

McNair, D. M., Lorr, M., and Droppleman, L. F. (1992). Profile of Mood States Manual. San Diego: Educational and Industrial Testing Service.

Moher, D., Schulz, K. F., and Altman, D. G. (2005). The CONSORT statenent: Revised Recommendations For Improving the Quality of Reports of ParallelGroup Randomized Trials. Zhongguo Zhong Xi Yi Jie He Za Zhi Zhongguo Zhongxiyi Jiehe Zazhi Chin. J. Integrated Tradit. Western Med. 25, 658. doi: 10.1186/1471-2288-1-2

Oh, H. A., Kim, D.-E., Choi, H. J., Kim, N. J., and Kim, D.-H. (2015). Anti-fatigue Effects of 20(S)-Protopanaxadiol and 20(S)-Protopanaxatriol in Mice. Pharmaceut. Soc. Japan 38, 1415-1419. doi: 10.1248/bpb.b15-00230

Pan, H. S., Zhao, Y., and Feng, Y. C. (2009). Effects of Ginsenosides and Ginsenoside Rgl on Central Neurotransmitter in the Exercise-induced Fatigue Rats. J. Jilin Institute Phys. Educ. 25, 57-59. doi: 10.3435/ j.issn:16721365.2009.03.057

Pan, H. S., Lai, Q. Y., Feng, Y. C., Zhao, Y., and Zhao, Z. M. (2010a). Experimental Studies on the Mechanism of Exercise Oxidative Stress and the Effects of Ginsenoside Rb1 on Anti-fatigue. J. Hebei Institute Phys. Educ. 24, 68-70. doi: 10.3969/j.issn.1008-3596.2010.02.020

Pan, H. S., Zhang, Z. H., Zhao, Z. M., and Feng, Y. C. (2010b). Effects of Ginsenosides on the Structure and Functions of Rat Skeletal Muscle with Exercise-induced Fatigue. J. Guangzhou Sport U. 30, 90-93. doi: 10.13830/ j.cnki.cn44-1129/g8.2010.01.002

Perel, P., Roberts, I., Sena, E., Wheble, P., Briscoe, C., Sandercock, P., et al. (2007). Comparison of treatment effects between animal experiments and clinical trials: Systematic review. BMJ 334, 7586. doi: 10.1136/ bmj.39048.407928.BE

Piper, B. F., Dibble, S. L., Dodd, M. J., Weiss, M. C., Slaughter, R. E., and Paul, S. M. (1998). The revised Piper Fatigue Scale: psychometric evaluation in women with breast cancer. Oncol. Nurs. Forum 25, 677. doi: 10.1007/s11136-013-0434-5

Qi, B., Liu, L., Zhang, H., Zhou, G. X., Wang, S., Duan, X. Z., et al. (2014). Antifatigue effects of proteins isolated from Panax quinquefolium. $J$. Ethnopharmacol. 153, 430-434. doi: 10.1016/j.jep.2014.02.045

Radad, K., Gille, G., Liu, L., and Rausch, W. D. (2006). Use of ginseng in medicine with emphasis on neurodegenerative disorders. J. Pharmacol. Sci. 100, 175186. doi: 10.1254/jphs.CRJ05010X

Ramirez, F. D., Motazedian, P., Jung, R. G., Santo, P. D., and Hibbert, B. (2017). Methodological Rigor in Preclinical Cardiovascular Studies: Targets to Enhance Reproducibility and Promote Research Translation. Circ. Res. 120, CIRCRESAHA.117.310628. doi: 10.1161/CIRCRESAHA.117.310628
Shi, D. J., Huang, B. S., Cui, Q. M., Qi, X., Chen, Z. S., and Cui, C. B. (2016). Antifatigue efect of Black Ginseng on KM mice. J. Agric. Sci. Yanbian Univ. 38, 204-208+219. doi: 10.13478/j.cnki.jasyu.2016.03.004

Soldati, F., and Sticher, O. (1980). HPLC Separation and Quantitative Determination of Ginsenosidesfrom Panax ginseng,Panax quinquefolium and from Ginseng Drug Preparations. J. Med. Plants Res. 38, 348-357. doi: 10.1055/s-2008-1074929

Song, X. T., Zhang, J. Y., Meng, L. Y., Gao, F., Wu, X. G., and Zhang, K. (2013). Effect of ginseng three alcohol saponin on blood glucose, blood lactic acid and blood urea nitrogen in sports fatigue rats. Chin. J. Public Health Eng. 12, 359361. doi: 10. 2855/j.issn:1671-4199.2013.05.0359

Stewart, L. A., Clarke, M., Rovers, M., Riley, R. D., Simmonds, M., Stewart, G., et al. (2015). Preferred reporting items for systematic review and meta- analyses of individual participant data: the PRISMA-IPD Statement. JAMA 313, 16571665. doi: 10.1001/jama.2015.3656

Sun, B. S., Gu, L. J., Fang, Z. M., Wang, C., Wang, Z., Lee, M. R., et al. (2009). Simultaneous quantification of 19 ginsenosides in black ginseng developed from Panax ginseng by HPLC-ELSD. J. Pharmaceut. Biomed. Anal. 50, 15-22. doi: 10.1016/j.jpba.2009.03.025

Twisk, F. A.-O., and Corsius, L. (2018). Cognitive-behavioural therapy for chronic fatigue syndrome: neither efficacious nor safe. Br. J. Psychiatry, 211, 259-261. doi: 10.1192/bjp.2018.136

Voshaar, M. A. H., Oude Klooster, P. M., Ten Christina, B., Vonkeman, H. E., Glas, C., Tim, J., et al. (2015). Assessment of Fatigue in Rheumatoid Arthritis: A Psychometric Comparison of Single-item, Multiitem, and Multidimensional Measures. J. Rheumatol. 42, 413-420. doi: 10.3899/jrheum.140389

Wang, J., Li, S. S., Fan, Y. Y., Chen, Y., Liu, D., Cheng, H.R., et al. (2010). Anti-fatigue activity of the water-soluble polysaccharides isolated from Panax ginseng C.A. Meyer. J. Ethnopharmacol. 130, 421-423. doi: 10.1016/j.jep.2010.05.027

Wang, J., Sun C., Zheng, Y., Zheng Y., Pan, H., Pan, H., et al. (2013). The effective mechanism of the polysaccharides from Panax ginseng on chronic fatigue syndrome. Arch. Pharmacal. Res. 37, 530-538. doi: 10.1007/s12272-013-0235-y

Wang, Y., Ma, L., Pei, S. P., and Cai, D. L. (2015).“Experimental study on Antifatigue Effect of ginsenoside Rg1”, in: Sixth National Conference on Integrated Chinese and Western Medicine Nutrition, Chongqing. p. 3.

Wang, H. P., Zhang, Y. B., Yang, X. W., Yang, X. B., Xu, W., Xu, F., et al. (2016). High-Performance Liquid Chromatography with Diode Array Detector and Electrospray Ionization Ion Trap Time-of-Flight Tandem Mass Spectrometry to Evaluate Ginseng Roots and Rhizomes from Different Regions. Molecules 21, 603-617. doi: 10.3390/molecules21050603

Whiting, P., Bagnall, A. M., Sowden, A. J., Cornell, J. E., Mulrow, C. D., and RamãRez, G. J. J. (2001). Interventions for the treatment and management of chronic fatigue syndrome: a systematic review. JAMA, 286, 1360-1368. doi: 10.1001/jama.286.11.1360

Xie, C. L., Gu, Y., Wang, W. W., Lu, L., Fu, D. L., Liu, A. J., et al. (2013). Efficacy and safety of Suanzaoren decoction for primary insomnia: a systematic review of randomized controlled trials. BMC Complement. Altern. Med. 13, 1-12. doi: $10.1186 / 1472-6882-13-18$

Xu, J. W., Zhou, Y., and Wang, D. L. (2010). Experimental Study on Ginsenoside $\mathrm{Rb} 1$ Against Exercise-Induced Skeletal Muscle Fatigue. J. Liaoning Med. Univ. 31, 111-113+125. doi: 10.3969/j.issn.1674-0424.2010.02.005

Xu, Y. F., Zhao, Y., Xing, N. N., Yang, F., and Lin, Y. L. (2011). Study on antifatigue effects of ginseng protein on mice. Sci. Technol. Food Industry 32, 406407+436. doi: 10.13386/j.issn.1002-0306.2011.11.025

Yang, Q. Y., Lai, X. D., Ouyang, J., and Yang, J. D. (2018). Effects of Ginsenoside Rg3 on fatigue resistance and SIRT1 in aged rats. Toxicology, 01, 409-432. doi: 10.1016/j.tox.2018.08.010

Yao, J. C. (2016). Efficacy of ginsenoside on anti-fatigue in mice. Clin. J. Chin. Med. 8, 9-10. doi: 10.3969/j.issn.1674-7860.2016.29.003

Yennurajalingam, S., Tannir, N. M., Williams, J. L., Lu, Z., Hess, K. R., FrisbeeHume, S., et al. (2017). A Double-Blind, Randomized, Placebo-Controlled Trial of Panax Ginseng for Cancer-Related Fatigue in Patients With Advanced Cancer. J. Natl. Compr. Cancer Netw. 15, 1111-1120. doi: 10.6004/jnccn.2017.0149

Zhao, Y., Dai, J. L., Lv, J., and Jia, T. Z. (2014). Compare Ginseng and Its Processed Products' Effect on Mice's Hypoxia and Anti-Fatigue Function. Liaoning J. Tradit. Chin. Med. 41, 1040-1042. doi: 10.13192/j.issn.1000-1719.2014.05.090

Zheng, Q., Bao, X. Y., Zhu, P. C., Tong, Q., Zheng, G. A.-O., and Wang, Y. A.-O. (2017). Ginsenoside Rb1 for Myocardial Ischemia/Reperfusion Injury: 
Preclinical Evidence and Possible Mechanisms. Oxid. Med. Cell. Longevity 2, 114. doi: $10.1155 / 2017 / 6313625$

Zheng, Q., Zhu, J. Z., Bao, X. Y., Zhu, P. C., Tong, Q., Huang, Y. Y., et al. (2018). A Preclinical Systematic Review and Meta-Analysis of Astragaloside IV for Myocardial Ischemia/Reperfusion Injury. Front. Physiol. 9, 795. doi: 10.3389/ fphys.2018.00795

Zhou, X. L., Xu, M. B., Jin, T. Y., Rong, P. Q., Zheng, G. Q., and Lin, Y. (2019). Preclinical Evidence and Possible Mechanisms of Extracts or Compounds from Cistanches for Alzheimer's Disease. Aging Dis. 10, 1075. doi: 10.14336/ AD.2018.0815-1
Conflict of Interest: The authors declare that the research was conducted in the absence of any commercial or financial relationships that could be construed as a potential conflict of interest.

Copyright $\odot 2020$ Jin, Rong, Liang, Zhang, Zheng and Lin. This is an open-access article distributed under the terms of the Creative Commons Attribution License (CC $B Y$ ). The use, distribution or reproduction in other forums is permitted, provided the original author(s) and the copyright owner(s) are credited and that the original publication in this journal is cited, in accordance with accepted academic practice. No use, distribution or reproduction is permitted which does not comply with these terms. 